\title{
Sensitivity analysis of six soil organic matter models applied to the decomposition of animal manures and crop residues
}

\author{
Daniele Cavalli, Pietro Marino Gallina, Luca Bechini \\ Department of Agricultural and Environmental Sciences - Production, Landscape, Agroenergy, \\ University of Milano, Italy
}

\begin{abstract}
Two features distinguishing soil organic matter simulation models are the type of kinetics used to calculate pool decomposition rates, and the algorithm used to handle the effects of nitrogen $(\mathrm{N})$ shortage on carbon (C) decomposition. Compared to widely used first-order kinetics, Monod kinetics more realistically represent organic matter decomposition, because they relate decomposition to both substrate and decomposer size. Most models impose a fixed $\mathrm{C}$ to $\mathrm{N}$ ratio for microbial biomass. When $\mathrm{N}$ required by microbial biomass to decompose a given amount of substrate-C is larger than soil available N, carbon decomposition rates are limited proportionally to $\mathrm{N}$ deficit (N inhibition hypothesis). Alternatively, C-overflow was proposed as a way of getting rid of excess $\mathrm{C}$, by allocating it to a storage pool of polysaccharides. We built six models to compare the combinations of three decomposition kinetics (first-order, Monod, and reverse Monod), and two ways to simulate the effect of $\mathrm{N}$ shortage on $\mathrm{C}$ decomposition ( $\mathrm{N}$ inhibition and $\mathrm{C}$-overflow). We conducted sensitivity analysis to identify model parameters that mostly affected $\mathrm{CO}_{2}$ emissions and soil mineral $\mathrm{N}$ during a simulated 189-day laboratory incubation assuming constant water content and temperature. We evaluated model outputs sensitivity at different
\end{abstract}

Correspondence: Daniele Cavalli, Department of Agricultural and Environmental Sciences - Production, Landscape, Agroenergy, University of Milano, via G. Celoria 2, 20133 Milano Italy.

Tel.: +39.02.503.16592 - Fax: +39.02 .503 .16575 .

E-mail: daniele.cavalli@unimi.it

Key words: Mineralisation; immobilisation; Monod kinetics; MichaelisMenten kinetics; overflow; priming effect.

Acknowledgements: work carried out in the project CN-MIP, co-funded by The Italian Ministry of Agriculture (Mipaaf) within the Multi-partner Call on Agricultural Greenhouse Gas Research (FACCE-JPI).

Conference presentation: SIA XLIV Congress, Bologna, 2015.

Received for publication: 1 February 2016.

Revision received: 14 May 2016.

Accepted for publication: 14 May 2016.

(C) Copyright D. Cavalli et al., 2016

Licensee PAGEPress, Italy

Italian Journal of Agronomy 2016; 11:757

doi:10.4081/ija.2016.757

This article is distributed under the terms of the Creative Commons Attribution Noncommercial License (by-nc 4.0) which permits any noncommercial use, distribution, and reproduction in any medium, provided the original author(s) and source are credited. stages of organic matter decomposition in a soil amended with three inputs of increasing $\mathrm{C}$ to $\mathrm{N}$ ratio: liquid manure, solid manure, and low- $\mathrm{N}$ crop residue.

Only few model parameters and their interactions were responsible for consistent variations of $\mathrm{CO}_{2}$ and soil mineral $\mathrm{N}$. These parameters were mostly related to microbial biomass and to the partitioning of applied $\mathrm{C}$ among input pools, as well as their decomposition constants. In addition, in models with Monod kinetics, $\mathrm{CO}_{2}$ was also sensitive to a variation of the half-saturation constants.

C-overflow enhanced pool decomposition compared to $\mathrm{N}$ inhibition hypothesis when $\mathrm{N}$ shortage occurred. Accumulated $\mathrm{C}$ in the polysaccharides pool decomposed slowly; therefore model outputs were not sensitive to a variation of its decay constant.

Six-month organic matter decomposition was generally higher for models implementing classical Monod kinetics, followed by models with first-order and reverse Monod kinetics, due to the effect of soil microbial biomass growth on decomposition rates. Moreover, models implementing Monod kinetics predicted positive priming effects of native organic matter after soil amendment, according to co-metabolism theory. Thus, priming was proportional to the increase of the microbial biomass and in turn to the decomposability of applied organic matter. We conclude that model calibration should focus only on the few important parameters.

\section{Introduction}

Soil organic matter (SOM) simulation models describe carbon (C) and nutrient - mainly nitrogen (N) - cycling in soil at different spatial (molecular to continental) and temporal (hours to decades) scales (Manzoni and Porporato, 2009). The use of SOM models (alone or integrated into more complex ecological, cropping and farming system models) spans many fields of application: e.g., the interpretation of SOM dynamics at micro-scale in laboratory incubation experiments (Van Veen et al., 1985; Blagodatsky et al., 1998), the estimation of longterm C dynamics in soils (Gijsman et al., 2002; Grace et al., 2006; Izaurralde et al., 2006), the quantification of $\mathrm{N}$ availability for plants (Coucheney et al., 2015) and $\mathrm{N}$ losses in the environment (Acutis et al., 2000), and the study of how SOM mineralization changes in response to a change of environmental variables (Davidson and Janssens, 2006; Conant et al., 2011; Liang et al., 2015; Sierra et al., 2015b).

Many SOM models are compartmental because they represent SOM heterogeneity through discrete pools, defined as homogeneous-in terms of composition and accessibility-fractions of SOM (Manzoni and Porporato, 2009; Sierra et al., 2011; Sierra and Müller, 2015). The number of pools varies from few to many. For example, in the ICBM model (Kätterer and Andrén, 2001) there are two pools, one of resistant (humified) and one of fresh organic matter, while in the BACWAVEWEB model (Zelenev et al., 2006) there are many pools of plant 
residues, microbial biomass, microfauna and native soil organic matter. Model pools are connected through a network of transformation/ decomposition fluxes that describe $\mathrm{C}$ and $\mathrm{N}$ turnover in soil. In the majority of the models (Manzoni and Porporato, 2009), SOM decomposition is simulated with first-order decomposition kinetics with respect to the decomposing pool (substrate). Instead, fewer models take simultaneously into account of the size of both the substrate and the microbial biomass (as a whole pool, an active fraction or as an exoenzymes pool) in simulating the SOM decomposition rate (Manzoni and Porporato, 2009). These models often assume that SOM decomposition is saturated with respect to substrate availability (Blagodatsky and Richter, 1998; Moorhead and Sinsabaugh, 2006; Ingwersen et al., 2008; Wutzler and Reichstein, 2008; Allison et al., 2010) or with respect to decomposers (Hadas et al., 1998; Schimel and Weintraub, 2003; Wutzler and Reichstein, 2008; Lawrence et al., 2009; Wutzler and Reichstein, 2013) using classical and reverse Monod (or MichaelisMenten) equations, respectively.

Models in which decomposition is co-limited by substrate and decomposers have been used in simulating $\mathrm{C}$ priming effects in soils (Blagodatsky et al., 1998; Neill and Gignoux, 2006; Blagodatsky et al., 2010; Wutzler and Reichstein, 2013), i.e., a change in microbial biomass activity (apparent priming effects) or in the turnover of SOM (real priming effects) due to addition of fresh (exogenous) organic matter or nutrient to soil (Kuzyakov et al., 2000; Blagodatskaya and Kuzyakov, 2008; Kuzyakov, 2010).

Another important aspect concerning the simulation of SOM turnover is related to the effect that shortage of $\mathrm{N}$ has on $\mathrm{C}$ decomposition. In many models, each pool is supposed to have a constant $\mathrm{C}$ to $\mathrm{N}$ ratio (i.e., pools are strictly homeostatic), and $\mathrm{N}$ decomposition follows C decomposition stoichiometrically (Manzoni and Porporato, 2009). Thus, organic $\mathrm{N}$ can be mineralized to, or additional $\mathrm{N}$ can be immobilised from, soil mineral $\mathrm{N}$ (SMN), depending on the $\mathrm{C}$ to $\mathrm{N}$ ratios of the substrate and the microbial biomass, and to the substrate- $\mathrm{C}$ use efficiency (the fraction of decomposed $\mathrm{C}$ assimilated by decomposers) (Verberne et al., 1990). According to Manzoni and Porporato (2009), when SMN requirement (the potential $\mathrm{N}$ immobilisation) exceeds $\mathrm{N}$ availability, two different approaches can be employed to guarantee homeostasis of decomposers: the $\mathrm{N}$ inhibition hypothesis and the $\mathrm{C}$ overflow mechanism. In the first case, a N-limitation factor is calculated such that $\mathrm{C}$ decomposition rates (and thus the overall SMN demand) are reduced, while in the second case, extra available- $\mathrm{C}$ is respired as $\mathrm{CO}_{2}$ (Schimel and Weintraub, 2003; Neill and Gignoux, 2006), or temporarily stored as extracellular polysaccharides (Hadas et al., 1998), without any reduction of SOM decomposition.

The implementation of Monod-type kinetics and the C-overflow mechanism increases model complexity and thus the number of parameters that must be initialized. Moreover, it is almost unavoidable that some parameters are subjected to calibration to achieve a good agreement between predictions and measurements (Müller et al., 2007). However, increasing model complexity can lead to undetermined model parameterisation and model overfitting (Sierra et al., 2015a). Thus, it is necessary to accurately select which model parameters have to be calibrated, according to available datasets. Parameters to be calibrated are normally those that have a major impact on the output variables of the model. To identify such parameters, sensitivity analysis (SA) can be used.

Sensitivity analysis studies how variation (uncertainty) in the output of a model can be apportioned to different sources of variation in model inputs (Saltelli et al., 2010). Sensitivity analysis is a necessary step of model development and a useful preliminary step to parameter calibration (Saltelli et al., 2004). Usually, SA requires running a model several times (the number of executions depending on the chosen method) by continuously changing the values of model parameters; these values are obtained by sampling parameters from pre-defined statistical distributions. Model outputs (resulting from the different model runs) are then analysed to assess the contribution of each parameter (and of their interactions) on model output variation. Among the different techniques for executing SA, global methods have been strongly recommended (Chan et al., 1997; Saltelli, 2002; Saltelli et al., 2004) because they are model-free (i.e., do not make any assumption about model structure and formulation) and evaluate model behaviour and output sensitivity in a wide range of parameters combinations (they are global). Global methods are suitable to rank parameters according to their sensitivity measures (Cariboni et al., 2007), and to select for calibration only the parameters to which model output is actually most sensitive (Cariboni et al., 2007; Saltelli et al., 2004). Examples of global SA of SOM models are not frequent (Cavalli and Bechini, 2011; Wang et al., 2005a, 2005b; Wang et al., 2013). Most published studies used simplified local methods, which change one parameter at a time in a specific point of the input space, while keeping the others constant (Schimel and Weintraub, 2003; Petersen et al., 2005a, 2005b).

Decomposition of organic input applied to soil is often studied with aerobic incubations conducted in the laboratory under constant temperature and soil water content. With such an approach it is possible to isolate unwanted environmental effects that affect SOM turnover and SMN dynamics. Results of laboratory aerobic incubations show that application of fresh organic matter to soil causes a temporary increase in $\mathrm{CO}_{2}$ emissions (Jensen et al., 2005; Morvan et al., 2006; Peters and Jensen, 2011); respiration of $\mathrm{C}$ usually follows a two-phase dynamic characterised by high emission rates in the first weeks and slower rates thereafter, when the more labile $\mathrm{C}$ in soil is exhausted. In soils amended with animal manures (Morvan et al., 2006; Bechini and Marino, 2009; Peters and Jensen, 2011) and low- $\mathrm{N}$ crop residues (Trinsoutrot et al., 2000; Jensen et al., 2005), net $\mathrm{N}$ immobilisation usually occurs immediately after amendment. The extent and duration of immobilisation depends mostly on the quality of added organic matter (in term of chemical recalcitrance, nutrient content and availability to microorganisms).

It is therefore very important to evaluate sensitivity of SOM decomposition at different time periods after soil amendment, and to consider organic inputs of different quality; this allows to identify the effect of model parameters on model output variation in relation to input quality, both in the short-term (about one-four weeks referring to laboratory conditions), medium-term (about four-eight weeks) and long-term (more than eight weeks).

The objective of this work was to modify a simple four-pool SOM model (ICBM/2BN, Kätterer and Andrén, 2001), replacing first-order with Monod-type decomposition kinetics, and introducing the C-overflow mechanism or the $\mathrm{N}$ inhibition hypothesis to limit $\mathrm{C}$ decomposition. Combining the modifications introduced in the original model, we had six variants of ICBM/2BN: a base model ( MOD $_{\text {First }}$, two variants that implement classical and reverse Monod kinetics instead of first-order kinetics $\left(\mathrm{MOD}_{\text {Monod }}\right.$ and $\mathrm{MOD}_{\text {RevMonod }}$ ), and three variants equal to the three previous models but that implement the C-overflow mechanism instead of $\mathrm{N}$ inhibition hypothesis $\left(\mathrm{MOD}_{\text {First+Over, }}\right.$ MOD $_{\text {Monod+Over }}$ and MOD RevMonod+0ver $_{\text {. }}$. The six models were subjected to global SA, highlighting the contribution of model parameters on the variation of $\mathrm{CO}_{2}$ emissions and $\mathrm{SMN}$ concentration at different stages of SOM decomposition, from 3 to 189 days after soil amendment with animal manures or crop residues, under constant soil temperature and soil water content. 


\section{Materials and methods}

\section{The six models}

\section{Model structure}

The base model (MOD ${ }_{\text {First }}$ ) builds on the ICBM/2BN model (Kätterer and Andrén, 2001), which represents soil organic matter with four pools: two pools of young organic matter (labile, $Y_{L}$, and resistant, $Y_{R}$ ), one of microbial biomass $(B)$ and one of old organic matter $(O)$. The structure of the base model is presented in Figure 1A, while model parameters and pools are listed in Table 1 and Table 2, respectively.

The base model represents young organic matter differently than Kätterer and Andrén (2001); in fact, each input (e.g., animal manures

Table 1. Parameters of the six models compared, and limits of parameters distributions used during sensitivity analysis.

\begin{tabular}{|c|c|c|c|c|c|c|c|}
\hline Parameter & Description* & Unit & Model $^{\circ}$ & Treatment ${ }^{\#}$ & $\begin{array}{l}\text { Limit } \\
\text { sent } \\
\text { ant }\end{array}$ & $\begin{array}{l}\text { ing } \\
y \\
y\end{array}$ & References $^{\S}$ \\
\hline & & & & & Min & Max & \\
\hline$f C_{Y R}$ soil & Fraction of $C_{\text {soil }}$ allocated to the pool $Y_{R}$ soil & $\mathrm{mg} \mathrm{Cmg} \mathrm{C}^{-1}$ & All & All & 0.01 & 0.10 & $h$ \\
\hline$k_{Y R}$ Soil & Decomposition constant of the pool $Y_{R}$ soil & $\begin{array}{l}\text { day }{ }^{-1} \\
M, M+0\end{array}$ & $\begin{array}{l}\mathrm{F}, \mathrm{F}+0, \mathrm{RM}, \mathrm{RM}+0 \\
\text { All }\end{array}$ & All & $\begin{array}{l}0.00042 \\
0.00059\end{array}$ & $\begin{array}{l}0.01500 \\
0.07000\end{array}$ & $\begin{array}{l}b, m, n \\
p\end{array}$ \\
\hline$k_{0}$ & Decomposition constant of the pool 0 & day ${ }^{-1}$ & $\begin{array}{l}\mathrm{F}, \mathrm{F}+0, \mathrm{RM}, \mathrm{RM}+0 \\
\mathrm{M}, \mathrm{M}+0\end{array}$ & $\begin{array}{l}\text { All } \\
\text { All }\end{array}$ & $\begin{array}{l}0.000057 \\
0.002488\end{array}$ & $\begin{array}{l}0.000219 \\
0.010621\end{array}$ & $\begin{array}{l}b, j, m \\
p\end{array}$ \\
\hline$f N_{B}$ & Fraction of initial $O N_{\text {soil }}$ allocated to the pool $B$ & $\mathrm{mg} \mathrm{N} \mathrm{mg} \mathrm{N}{ }^{-1}$ & All & All & 0.02 & 0.05 & $a, d, e, f, 0$ \\
\hline eff & Substrate C use efficiency & $\mathrm{mg} \mathrm{Cmg} \mathrm{C}^{-1}$ & All & All & 0.4 & 0.8 & $b, j, m, n$ \\
\hline$f_{B}$ & Recycled fraction of microbial residues & $\mathrm{mg} \mathrm{C} \mathrm{mg} \mathrm{C}^{-1}$ & All & All & 0.60 & 0.95 & $j$ \\
\hline$k_{B}$ & Decomposition constant of the pool $B$ & day $^{-1}$ & All & All & 0.0073 & 0.0219 & $b, m, n$ \\
\hline$f C_{Y L}$ & Fraction of $C_{\text {input }}$ allocated to the pool $Y_{L}$ & $\mathrm{mg} \mathrm{Cmg} \mathrm{C}^{-1}$ & All & $\begin{array}{l}\mathrm{LM}, \mathrm{SM} \\
\mathrm{CR}\end{array}$ & $\begin{array}{l}0.18 \\
0.05\end{array}$ & $\begin{array}{l}0.65 \\
0.20\end{array}$ & $b, c, i, k, l, n$ \\
\hline$f C_{Y R}$ & Fraction of $C_{\text {input }}$ allocated to the pool $Y_{R}$ & $\mathrm{mg} \mathrm{Cmg} \mathrm{C}^{-1}$ & All & LM, SM & 0.14 & 0.76 & \\
\hline & & & & CR & 0.65 & 0.92 & $f C_{Y R}=1-f C_{Y L}-f C_{Y H L}$ \\
\hline$f C_{Y H L}$ & Fraction of $C_{\text {input }}$ allocated to the pool $Y_{H L}$ & $\mathrm{mg} \mathrm{C} \mathrm{mg} \mathrm{C}^{-1}$ & All & $\begin{array}{l}\text { LM, SM } \\
\text { CR }\end{array}$ & $\begin{array}{l}0.06 \\
0.03\end{array}$ & $\begin{array}{l}0.21 \\
0.15\end{array}$ & $b, c, i, k, l, n$ \\
\hline$f N_{Y L}$ & Fraction of $O N_{\text {input }}$ allocated to the pool $Y_{L}$ & $\mathrm{mg} \mathrm{N} \mathrm{mg} \mathrm{N} \mathrm{N}^{-1}$ & All & $\begin{array}{l}\text { LM, SM } \\
\text { CR }\end{array}$ & $\begin{array}{l}0.48 \\
0.23\end{array}$ & $\begin{array}{l}0.65 \\
0.45\end{array}$ & $b, c, i, k, l, n$ \\
\hline$f N_{Y R}$ & Fraction of $O N_{\text {input }}$ allocated to the pool $Y_{R}$ & $\mathrm{mg} \mathrm{Nmg} \mathrm{N}^{-1}$ & All & $\begin{array}{l}\text { LM } \\
\text { SM } \\
\text { CR }\end{array}$ & $\begin{array}{l}0.17 \\
0.01 \\
0.03\end{array}$ & $\begin{array}{l}0.47 \\
0.42 \\
0.67\end{array}$ & $f N_{Y R}=1-f N_{Y L}-f N_{Y H L}$ \\
\hline$f N_{Y H L}$ & Fraction of $O N_{\text {input }}$ allocated to the pool $Y_{H L}$ & $\mathrm{mg} \mathrm{N} \mathrm{mg} \mathrm{N} \mathrm{N}^{-1}$ & All & $\begin{array}{l}\text { LM } \\
\text { SM } \\
\text { CR }\end{array}$ & $\begin{array}{l}0.05 \\
0.10 \\
0.10\end{array}$ & $\begin{array}{l}0.18 \\
0.34 \\
0.52\end{array}$ & $\begin{array}{l}f N_{Y H L}=f C_{Y H L} \times\left(C_{\text {input }}\right. \\
\left.10 N_{\text {input }}\right) / 15\end{array}$ \\
\hline$k_{Y L}$ & Decomposition constant of the pool YL & $\mathrm{day}^{-1}$ & $\mathrm{~F}, \mathrm{~F}+0, \mathrm{RM}, \mathrm{RM}+\mathrm{OM}, \mathrm{M}+0$ & $\begin{array}{l}\text { All } \\
\text { All }\end{array}$ & $\begin{array}{l}0.0151 \\
0.0118\end{array}$ & $\begin{array}{l}0.4200 \\
1.1963\end{array}$ & $b, n p$ \\
\hline$k_{Y R}$ & Decomposition constant of the pool $Y_{R}$ & $\mathrm{day}^{-1}$ & $\mathrm{~F}, \mathrm{~F}+0, \mathrm{RM}, \mathrm{RM}+\mathrm{OM}, \mathrm{M}+0$ & $\begin{array}{l}\text { All } \\
\text { All }\end{array}$ & $\begin{array}{l}0.00042 \\
0.00059\end{array}$ & $\begin{array}{l}0.01500 \\
0.07000\end{array}$ & $b, m, n p$ \\
\hline$k_{Y H L}$ & Decomposition constant of the pool $Y_{H L}$ & $\mathrm{day}^{-1}$ & $\mathrm{~F}, \mathrm{~F}+0, \mathrm{RM}, \mathrm{RM}+\mathrm{OM}, \mathrm{M}+0$ & $\begin{array}{l}\text { Al } \\
\text { IAll } \\
\end{array}$ & $\begin{array}{l}0.000057 \\
0.000016\end{array}$ & $\begin{array}{l}0.000219 \\
0.000235\end{array}$ & $b, n p$ \\
\hline$k_{P}$ & Decomposition constant of the pool $P$ & day ${ }^{-1}$ & $\mathrm{~F}+0, \mathrm{RM}+\mathrm{OM}+0$ & $\begin{array}{l}\text { All } \\
\text { All }\end{array}$ & $\begin{array}{l}0.001200 \\
0.000005\end{array}$ & $\begin{array}{l}0.013500 \\
0.002135\end{array}$ & $g p$ \\
\hline$k_{r m}$ & $\begin{array}{l}\text { Half-saturation constant for fluxes from } \\
Y_{R} \text { soil, } Y_{L}, Y_{R}, Y_{H L}, O \text { and } P \text { to } B\end{array}$ & $\mathrm{mg} \mathrm{C} \mathrm{kg}^{-1}$ & $\mathrm{RM}, \mathrm{RM}+0$ & All & 89 & 1328 & $g$ \\
\hline$k_{m Y R}$ soil & Half-saturation constant for the flux from $Y_{R}$ soil to $B$ & $\mathrm{mg} \mathrm{C} \mathrm{kg}^{-1}$ & $\mathrm{M}, \mathrm{M}+0$ & All & 125 & 6202 & $q$ \\
\hline$k_{m 0}$ & Half-saturation constant for the flux from 0 to $B$ & $\mathrm{mg} \mathrm{C} \mathrm{kg}^{-1}$ & $\mathrm{M}, \mathrm{M}+0$ & All & 3897 & 64394 & $q$ \\
\hline$k_{m Y L}$ & Half-saturation constant for the flux from $Y_{L}$ to $B$ & $\mathrm{mg} \mathrm{C} \mathrm{kg}^{-1}$ & $\mathrm{M}, \mathrm{M}+0$ & All & 69 & 3782 & $q$ \\
\hline$k_{m Y R}$ & Half-saturation constant for the flux from $Y_{R}$ to $B$ & $\mathrm{mg} \mathrm{C} \mathrm{kg}^{-1}$ & $\mathrm{M}, \mathrm{M}+0$ & All & 125 & 6202 & $q$ \\
\hline$k_{m Y H L}$ & Half-saturation constant for the flux from $Y_{H L}$ to $B$ & $\mathrm{mg} \mathrm{C} \mathrm{kg}^{-1}$ & $\mathrm{M}, \mathrm{M}+0$ & All & 25 & 1427 & $q$ \\
\hline$k_{m P}$ & Half-saturation constant for the flux from $P$ to $B$ & $\mathrm{mg} \mathrm{C} \mathrm{kg-1}$ & $\mathrm{M}+0$ & All & 1 & 210 & $q$ \\
\hline
\end{tabular}

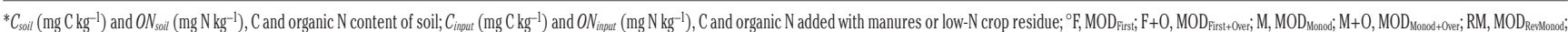

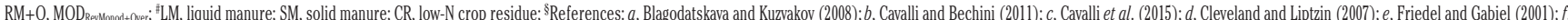
Griffiths et al. (2012); $g$, Hadas et al. (1998); $h$, Hadas et al. (2004); $i$, Henriksen and Breland (1999); $j$, Kätterer and Andrén (2001); $k$, Morvan et al. (2006); $l$, Peters and Jensen (2011); $m$, Petersen et al. (2005a); $n$, Petersen et al. (2005b); 0 , Wutzler and Reichstein (2013); p, calculated according to Eq. 10; $q$, calculated according to Eq. 11. 
and crop residues) is allocated to three young pools: a labile and a resistant one $\left(Y_{L}\right.$ and $\left.Y_{R}\right)$ as in ICBM/2BN, plus a very resistant pool $\left(Y_{H L}\right)$. This additional pool represents the lignified fraction of crop residues (Henriksen and Breland, 1999; Hadas et al., 2004) and the partially humified organic matter of animal manures (Petersen et al., $2005 \mathrm{~b})$. Thus, a fraction of input-C is assigned to the labile pool $\left(f C_{Y L}\right)$, another to the humus-lignin like pool $\left(Y_{H L}\right)$, and the remaining to the resistant pool $\left(f C_{Y R}=1-f C_{Y L}-f C_{0}\right)$. Similarly, input organic $\mathrm{N}$ is partitioned among the three young pools with the parameters $f N_{Y L}, f N_{Y R}$ and $f N_{Y H L}$. In the base model, soil (without added inputs: grey boxes in Figure 1A) is supposed to have some organic matter not only in the old pool $(O)$ but also in the young resistant pool $\left(Y_{R}\right.$ soil $)$, due to soil disturbance (Hadas et al., 2004).

Connections (decomposition and transformation fluxes) among model pools are presented in Figure 1A. Microbial biomass feeds on both young and resistant pools; a fraction $\left(f_{B}\right)$ of microbial residues is recycled through the living microbial biomass, while the remaining fraction $\left(1-f_{B}\right)$ is assimilated to the pool $O$. During organic matter decomposition, microbial biomass assimilates only a fraction (eff) of the incoming $\mathrm{C}$, and the other part $(1-$ eff $)$ is released as $\mathrm{CO}_{2}$.
We modified the base model for the decomposition kinetics and the effects of $\mathrm{N}$ shortage on pool decomposition (Table 3).

\section{Decomposition of organic pools}

In the base model, all pools decay with first-order kinetics with respect to the decomposing pool. The outflow of $\mathrm{C}$ from each pool (pool decomposition rate, $D E C$ ) depends on the size of the substrate pool $\left(C_{S}\right)$ and on its decomposition constant $(k)$ :

$$
D E C=k \times C_{S}
$$

A first model modification ( MOD $_{\text {Monod }}$ ) assumes that decomposition of substrates $C_{S}$ depends also on the size of the microbial biomass $\left(C_{B}\right)$ pool, according to a classical formulation of the Monod equation (Blagodatsky et al., 1998):

$$
D E C=k^{\prime} \times C_{B} \times \frac{C_{S}}{k_{m}+C_{S}}
$$

\begin{tabular}{|c|c|c|c|c|}
\hline Model pool & State variable & Description & Unit & Calculated value* \\
\hline$Y_{R}$ soil (soil resistant organic matter) & $\begin{array}{l}C_{Y R} \text { soil } \\
N_{Y R} \text { soil }\end{array}$ & $\begin{array}{l}\mathrm{C} \text { content of } Y_{R} \text { soil } \\
\mathrm{N} \text { content of } Y_{R} \text { soil }\end{array}$ & $\begin{array}{l}\text { mg C kg-1 } \\
\mathrm{mg} \mathrm{N} \mathrm{kg}^{-1}\end{array}$ & $\begin{array}{c}f C_{Y R} \text { soil } \times C_{\text {soil }} \\
C_{Y R} /\left(C_{\text {soil }} / O N_{\text {soil }}\right)\end{array}$ \\
\hline$B$ (soil microbial biomass) & $\begin{array}{l}C_{B} \\
N_{B}\end{array}$ & $\begin{array}{l}\text { C content of } B \\
\text { N content of } B\end{array}$ & $\begin{array}{l}\mathrm{mg} \mathrm{C} \mathrm{kg}^{-1} \\
\mathrm{mg} \mathrm{N} \mathrm{kg}^{-1}\end{array}$ & $\begin{array}{c}C_{\text {soil }} \times 0.02 \\
f N_{B} \times 0 N_{\text {soil }}\end{array}$ \\
\hline$O$ (soil old organic matter) & $\begin{array}{l}C_{0} \\
N_{0}\end{array}$ & $\begin{array}{l}\mathrm{C} \text { content of } O \\
\mathrm{~N} \text { content of } O\end{array}$ & $\begin{array}{l}\mathrm{mg} \mathrm{C} \mathrm{kg-1} \\
\mathrm{mg} \mathrm{N} \mathrm{kg}^{-1}\end{array}$ & $\begin{array}{c}C_{\text {soil }}-C_{Y R}-C_{B} \\
O N_{\text {soil }}-N_{Y R}-N_{B}\end{array}$ \\
\hline$Y_{L}$ (added labile organic matter) & $\begin{array}{l}C_{Y L} \\
N_{Y L}\end{array}$ & $\begin{array}{l}\mathrm{C} \text { content of } Y_{L} \\
\mathrm{~N} \text { content of } Y_{L}\end{array}$ & $\begin{array}{l}\mathrm{mg} \mathrm{C} \mathrm{kg} \\
\mathrm{mg} \mathrm{N} \mathrm{kg}^{-1}\end{array}$ & $\begin{array}{l}f C_{Y L} \times C_{\text {input }} \\
f N_{Y L} \times O N_{\text {input }}\end{array}$ \\
\hline$Y_{R}$ (added resistant organic matter) & $\begin{array}{l}C_{Y R} \\
N_{Y R}\end{array}$ & $\begin{array}{l}\mathrm{C} \text { content of } Y_{R} \\
\mathrm{~N} \text { content of } Y_{R}\end{array}$ & $\begin{array}{l}\mathrm{mg} \mathrm{C} \mathrm{kg} \\
\mathrm{mg} \mathrm{N} \mathrm{kg}\end{array}$ & $\begin{array}{c}f C_{Y R} \times C_{\text {input }} \\
f N_{Y R} \times O N_{\text {input }}\end{array}$ \\
\hline$Y_{H L}$ (added humified or lignified organic matter) & $\begin{array}{l}C_{Y H L} \\
N_{Y H L}\end{array}$ & $\begin{array}{l}\mathrm{C} \text { content of } Y_{H L} \\
\mathrm{~N} \text { content of } Y_{H L}\end{array}$ & $\begin{array}{l}\mathrm{mg} \mathrm{C} \mathrm{kg-1} \\
\mathrm{mg} \mathrm{N} \mathrm{kg}^{-1}\end{array}$ & $\begin{array}{c}f C_{Y H L} \times C_{\text {input }} \\
f N_{Y H L} \times O N_{\text {input }}\end{array}$ \\
\hline
\end{tabular}

Table 2. Initial state of pools of the six models compared.

${ }^{*} C_{\text {soil }}\left(\mathrm{mg} \mathrm{C} \mathrm{kg}^{-1}\right)$ and $O N_{\text {soil }}\left(\mathrm{mg} \mathrm{N} \mathrm{kg}^{-1}\right), \mathrm{C}$ and organic $\mathrm{N}$ content of soil; $C_{\text {input }}\left(\mathrm{mg} \mathrm{C} \mathrm{kg}^{-1}\right)$ and $O N_{\text {ipout }}\left(\mathrm{mg} \mathrm{N} \mathrm{kg}^{-1}\right), \mathrm{C}$ and organic $\mathrm{N}$ added with manures or low-N crop residue.

A

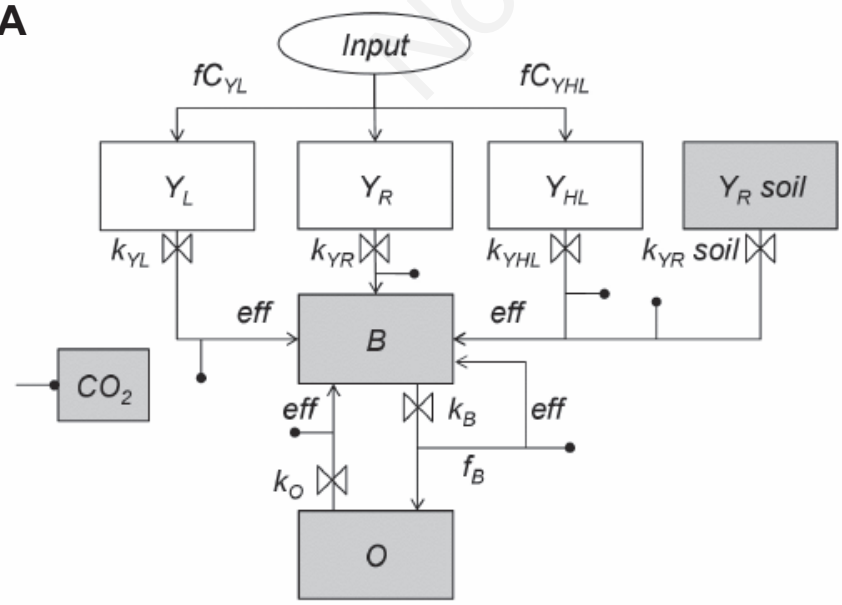

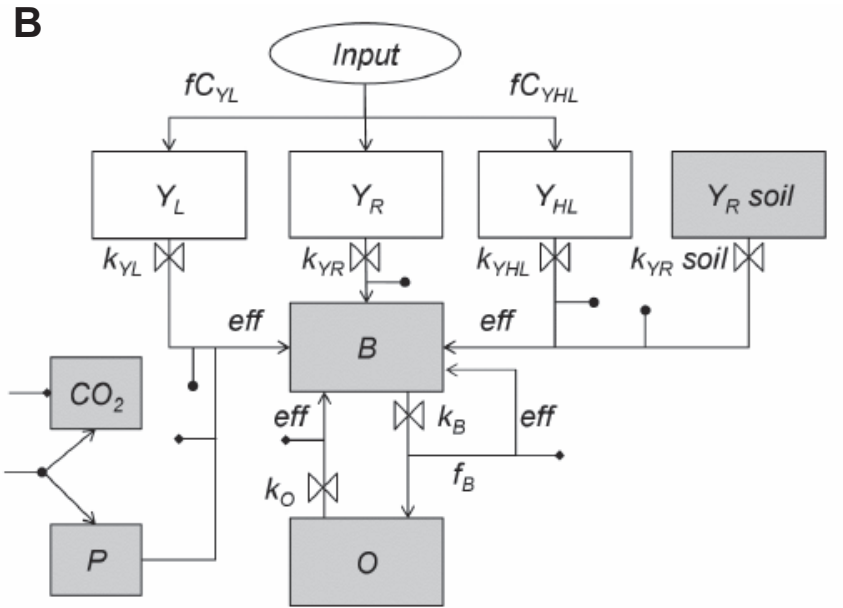

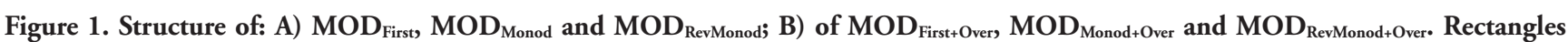
represent $\mathrm{C}$ pools, valves represent $\mathrm{C}$ rates, arrows represent $\mathrm{C}$ flows, and dot-ended arrows represent $\mathrm{CO}_{2}$ emissions or $\mathrm{C}$ flow to the polysaccharide pool. For a description of model pools and parameters refer to Table 1 and Table 2. 
where $k^{\prime}$ is the decomposition constant, $k_{m}$ is the half-saturation constant which indicates the size of $C_{S}$ at which $D E C$ proceeds at half rate compared to first-order.

Alternatively, a second model modification ( $\left.\mathrm{MOD}_{\text {RevMonod }}\right)$ is based on a reverse Monod equation (Eq. 3), which assumes that $C_{S}$ is sufficiently large, and that microbial biomass-rather than the substrate-saturates decomposition (Hadas et al., 1998):

$$
D E C=k \times C_{S} \times \frac{C_{B}}{k_{r m}+C_{B}}
$$

where $k_{r m}$ is the half-saturation constant for the reversed Monod models.

\section{Nitrogen limitation on decomposition}

In all models, all pools are constrained to have a defined and constant $\mathrm{C}$ to $\mathrm{N}$ ratio; thus, microorganisms are considered homeostatic (Manzoni and Porporato, 2009). With this assumption, N decomposition follows $\mathrm{C}$ decomposition stoichiometrically, and $\mathrm{N}$ in each flux is equal to $D E C$ divided by the $\mathrm{C}$ to $\mathrm{N}$ ratio of the decomposing pool. Moreover, the entire $\mathrm{N}$ in the flux (organic $\mathrm{N}$ ) is considered available for microorganisms, according to the direct hypothesis for microbial $\mathrm{N}$ immobilisation (Manzoni and Porporato, 2009).

Considering that the $\mathrm{C}$ to $\mathrm{N}$ ratio of the receiving pool (e.g., microbial biomass, $C / N_{B}$ ) can be different from the $\mathrm{C}$ to $\mathrm{N}$ ratio of the decomposing pool $\left(C / N_{S}\right)$, and that the $\mathrm{C}$ entering the receiving pool is different than the $\mathrm{C}$ leaving the decomposing pool, the $\mathrm{N}$ balance $(\Delta N)$ of each flux is calculated as (Verberne et al., 1990):

$$
\Delta N=D E C \times\left(\frac{1}{C / N_{S}}-\frac{e f f}{C / N_{B}}\right)
$$

where eff is the substrate assimilation efficiency (equal to one for the flux from $B$ to $O$ ).
The $\mathrm{C}$ to $\mathrm{N}$ ratio of microbial biomass and eff allow calculating the critical $\mathrm{C}$ to $\mathrm{N}$ ratio $\left(C / \mathrm{N}_{\text {crit }}\right)$ for microbial $\mathrm{N}$ immobilisation, which represents the substrate $\mathrm{C}$ to $\mathrm{N}$ ratio at which $\mathrm{N}$ immobilisation is equal to $\mathrm{N}$ mineralisation $(\Delta N=0)$ :

$$
C / N_{\text {crit }}=\frac{C / N_{B}}{\text { eff }}
$$

If $\Delta N$ is positive (Eq. 4), excess organic $\mathrm{N}$ is mineralised to ammonium, otherwise, if it is negative, the $\mathrm{N}$ required by the receiving pool is immobilised from the soil mineral $\mathrm{N}$ pool $(\mathrm{SMN}=$ ammonium- $\mathrm{N}+$ nitrate-N). Potential $\mathrm{N}$ immobilisation $\left(I_{\left.M M_{P O T}\right)}\right.$ from all $n$-fluxes requiring extra $\mathrm{N}$ is then calculated as:

$$
I M M_{P O T}=\max \left[0 ; \sum_{i=1}^{n}-\Delta N_{i}\right]
$$

We first implemented the effect of SMN shortage (i.e., when $I M M_{P O T}$ is higher than available SMN) according to the $\mathrm{N}$ inhibition hypothesis (Manzoni and Porporato, 2007): decomposition fluxes having a negative $\Delta N$ are limited by a reduction factor $\left(N_{\text {lim }}\right)$, ensuring that $I M M_{P O T}$ never exceeds SMN availability. In the definition of $N_{\text {lim }}$ we assume that the maximum amount of $\mathrm{N}$ that can be immobilised is equal to a fraction $\left(f_{\max }=0.95\right)$ of SMN, in order to avoid the complete depletion of SMN within one time step (Petersen et al., 2005b). The factor $N_{\text {lim }}$ is calculated as:

$N_{\text {lim }}=\min \left[1 ; \frac{\mathrm{SMN} \times f_{\max }}{I M M_{P O T}}\right]$

Thereafter, $D E C$ of all immobilising fluxes are multiplied by $N_{\text {lim }}$, thus reducing both $\mathrm{C}$ mineralization and SMN demand if $N_{\text {lim }}<1$.

Alternatively to the $\mathrm{N}$ inhibition hypothesis, three variants of the model $\left(\mathrm{MOD}_{\text {First+Over, }}\right.$ MOD $\mathrm{D}_{\text {Monod+Over }}$ and $\mathrm{MOD}_{\text {RevMonod+Over; }}$ Figure $1 \mathrm{~B}$ )

\begin{tabular}{|c|c|c|c|c|}
\hline \multirow[t]{2}{*}{ Model } & \multicolumn{2}{|c|}{ Decomposition kinetics } & \multicolumn{2}{|c|}{ Shortage of $\mathbf{N}$ effect on decomposition } \\
\hline & Type & Fluxes affected & Mechanism & Fluxes affected \\
\hline MOD $D_{\text {First }}$ & First-order & All fluxes & $\mathrm{N}$ inhibition & All fluxes \\
\hline $\mathrm{MOD}_{\text {Monod }}$ & $\begin{array}{l}\text { Classical Monod } \\
\text { First-order }\end{array}$ & $\begin{array}{l}Y_{L} \rightarrow B ; Y_{R} \rightarrow B ; Y_{H L} \rightarrow B ; Y_{R} \text { soil } \rightarrow B ; O \rightarrow B \\
B \rightarrow B ; B \rightarrow 0\end{array}$ & N inhibition & All fluxes \\
\hline $\mathrm{MOD}_{\text {RevMonod }}$ & $\begin{array}{l}\text { Reverse Monod } \\
\text { First-order }\end{array}$ & $\begin{array}{l}Y_{L} \rightarrow B ; Y_{R} \rightarrow B ; Y_{H L} \rightarrow B ; Y_{R} \text { soil } \rightarrow B ; O \rightarrow B \\
B \rightarrow B ; B \rightarrow 0\end{array}$ & $\mathrm{~N}$ inhibition & All fluxes \\
\hline $\mathrm{MOD}_{\text {First+Over }}$ & First-order & All fluxes & $\begin{array}{l}\mathrm{N} \text { inhibition } \\
\text { C-overflow }\end{array}$ & $\begin{array}{l}B \rightarrow B ; B \rightarrow 0 ; O \rightarrow B ; P \rightarrow B \\
Y_{L} \rightarrow B ; Y_{R} \rightarrow B ; Y_{H L} \rightarrow B ; Y_{R} \text { soil } \rightarrow B\end{array}$ \\
\hline $\mathrm{MOD}_{\text {Monod+Overflow }}$ & $\begin{array}{l}\text { Classical Monod } \\
\text { First-order }\end{array}$ & $\begin{array}{l}Y_{L} \rightarrow B ; Y_{R} \rightarrow B ; Y_{H L} \rightarrow B ; Y_{R} \text { soil } \rightarrow B ; O \rightarrow B ; P \rightarrow B \\
B \rightarrow B ; B \rightarrow O\end{array}$ & $\begin{array}{l}\mathrm{N} \text { inhibition } \\
\text { C-overflow }\end{array}$ & $\begin{aligned} B & \rightarrow B ; B \rightarrow O ; O \rightarrow B ; P \rightarrow B \\
Y_{L} & \rightarrow B ; Y_{R} \rightarrow B ; Y_{H L} \rightarrow B ; Y_{R} \text { soil } \rightarrow B\end{aligned}$ \\
\hline $\mathrm{MOD}_{\text {RevMonod+Overflow }}$ & $\begin{array}{l}\text { Reverse Monod } \\
\text { First-order }\end{array}$ & $\begin{array}{l}Y_{L} \rightarrow B ; Y_{R} \rightarrow B ; Y_{H L} \rightarrow B ; Y_{R} \text { soil } \rightarrow B ; O \rightarrow B ; P \rightarrow B \\
B \rightarrow B ; B \rightarrow 0\end{array}$ & $\begin{array}{l}\mathrm{N} \text { inhibition } \\
\text { C-overflow }\end{array}$ & 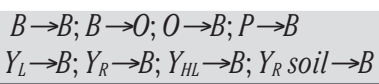 \\
\hline
\end{tabular}
implement the C-overflow mechanism (Manzoni and Porporato, 2009),

Table 3. Summary of decomposition kinetics and mechanisms of $\mathrm{N}$ control over decomposition implemented in the six models com-

\begin{tabular}{|c|c|c|c|c|c|c|}
\hline \multirow[t]{2}{*}{ Organic input } & \multicolumn{3}{|c|}{ Parameter* } & \multicolumn{3}{|c|}{ Application ( $\mathrm{mg} \mathrm{kg}^{-1}$ ) } \\
\hline & $\mathrm{C} / \mathrm{TN}$ & $\mathrm{C} / \mathrm{ON}$ & $\mathrm{NH}_{4}-\mathrm{N} / \mathrm{TN}$ & C & ON & $\mathrm{NH}_{4}-\mathrm{N}$ \\
\hline Liquid manure & 7 & 13 & 0.46 & 677 & 54 & 46 \\
\hline Solid manure & 19 & 25 & 0.24 & 1863 & 76 & 24 \\
\hline Low-N crop residue & 52 & 52 & 0.00 & 1200 & 23 & 0 \\
\hline
\end{tabular}
pared.

Table 4. Characteristics of the three organic inputs used in sensitivity analysis, and amounts of $\mathrm{C}$ and $\mathrm{N}$ applied to the soil.

*TN, total N; ON, organic N. 
only on the fluxes from young pools to $B$ (Hadas et al., 1998). According to this hypothesis, when shortage of SMN occurs, $D E C$ of immobilising fluxes is not limited by mineral $\mathrm{N}$ availability; instead, excess $\mathrm{C}$ is directed to polysaccharides $(P) \mathrm{C}$-pool (Hadas et al., 1998). Therefore, excess of $\mathrm{C}\left(D E C_{\text {overflow }}\right.$, i.e., $\mathrm{C}$ that cannot be assimilated in $B$ under the current SMN availability) in each flux is calculated as (Manzoni and Porporato, 2009):

$$
D E C_{\text {overflow }}=e f f \times D E C-C / N_{B} \times\left(\frac{D E C}{C / N_{S}}+I M M_{M A X}\right)
$$

where $I M M_{M A X}$ (equal to $-\Delta N \times N_{\text {lim }}$ ) is the maximum amount of SMN that can be immobilised. Therefore, decomposed $C$ assimilated by $B$ is higher compared to that assimilated under the $\mathrm{N}$ inhibition hypothesis. Decomposition of the polysaccharides pool is limited by $\mathrm{N}$ according to the $\mathrm{N}$ inhibition hypothesis (Table 3 ).

\section{Sensitivity analysis}

\section{Treatments simulated and model outputs recorded}

The six models underwent $\mathrm{SA}$ in order to assess the sensitivity of $\mathrm{C}$ and $\mathrm{N}$ decomposition to a variation of model parameters. For each model, we performed separate SAs for the following treatments: i) unamended (bare) control soil (CON); ii) liquid manure (LM)-amended soil; iii) solid manure (SM)-amended soil; iv) low-N crop residue (CR)amended soil (Table 4).

The selected model outputs of interest were $\mathrm{CO}_{2}$ and SMN concentration simulated in seven dates (after 3, 7, 14, 29, 44, 73, and 189 days after amendment). For amended treatments (LM, SM and CR), model outputs were calculated subtracting the values simulated in CON, to obtain the net effect.

Soil organic $\mathrm{C}$ and organic $\mathrm{N}$ were set to $11,065 \mathrm{mg} \mathrm{C} \mathrm{kg}^{-1}$ and 1086 $\mathrm{mg} \mathrm{N} \mathrm{kg}{ }^{-1}$, respectively, while inorganic soil $\mathrm{N}$ content was set to $7 \mathrm{mg}$ $\mathrm{N} \mathrm{kg}^{-1}$; these values were measured during an incubation experiment (unpublished data). The amount of $\mathrm{C}$ and $\mathrm{N}$ applied with LM, SM, and $\mathrm{CR}$ are reported in Table 4. Manures were applied at a rate of about 340 $\mathrm{kg} \mathrm{N} \mathrm{ha}{ }^{-1}$, according to national regulations, while their chemical composition was set according to Cavalli et al. (2015). Crop residue was applied considering a maize grain production of $12 \mathrm{t}$ dry matter (DM) $\mathrm{ha}^{-1}$, a grain DM to stalk DM ratio of 0.8 and a $\mathrm{C}$ concentration of maize stalk of $45 \%$. For both manures and crop residue, we considered an incorporation depth of $0.3 \mathrm{~m}$ and a soil bulk density of $1.2 \mathrm{~g} \mathrm{~cm}^{-3}$.

We have run the simulations of SA by assuming constant soil temperature and water content, and, no response function (Kätterer and Andrén, 2001) was implemented.

Therefore, as a combination of six models, four treatments, two model outputs, and seven dates, we performed 336 SAs.

\section{The method of Sobol'}

As mentioned in the introduction, SA requires running the model many times by changing the values of parameters (which are sampled from a pre-defined statistical distribution).

The method of Sobol' (Sobol', 1993; Saltelli et al., 2004; Sobol' and Kucherenko, 2005) is based on the decomposition of total model output variance $(V)$ into different sources of variation: i.e., the partial variances due to single parameters $\left(V_{i}\right)$, and all possible interactions of order $w$ among model parameters $\left(V_{i j}, V_{i j m}\right.$, etc. $)$ :

$$
V=\sum_{i} V_{i}+\sum_{i<j} V_{i j}+\sum_{i<j<m} V_{i j m}+\ldots+V_{12 \ldots w}
$$

where $i, j, m, \ldots$, and $w$ represent model parameters.

Sensitivity indices $(S)$ are then calculated as the ratios of condition- al variances $\left(V_{i}, V_{i j}\right.$, etc. $)$ to total (unconditional) variance $\left(S_{i}=V / N ; S_{i j}\right.$ $=V_{i j} N$, etc.), and represent the contribution to the total output variance of single parameters $\left(S_{i}\right)$ or combinations of parameters $\left(S_{i j}\right.$, etc.). Total-order indices (ST) represent the whole contribution of one parameter (alone and in combination with all other parameters) to total output variance (i.e., $S T_{i}$ is the sum of all terms in Eq. 9 involving parameter $i$, divided by $V$ ). Given the rather high number of model parameters (from 13 to 20 , Table 1), we calculated first-order $\left(S_{i}\right)$ and total-order $\left(S T_{i}\right)$ sensitivity indices only. Thus, the difference between $S T_{i}$ and $S_{i}$, estimates the fraction of total output variance due to interactions (from order 2 up to order $w$ ) between parameter $i$ and all other $w$-1 parameters.

Monte Carlo estimation of sensitivity indices was done according to Saltelli et al. (2010) using a sample size equal to $2^{16}$.

\section{Parameters distributions and models initialisation}

During SAs, model parameters were sampled from uniform distributions, due to the lack of detailed a priori information about parameters variability. Lower and upper boundaries of each distribution were derived from values of parameters of similar significance used in other models, experimental measurements, or both (Table 1). When more than one parameter value was available in the literature, we set the limits of the distribution according to the minimum and the maximum values found. Conversely, when only one value was found, we set the lower and upper boundary of the distribution as $\pm 50 \%$ of the reference value (Cavalli and Bechini, 2011).

Ranges of the decomposition and half-saturation constants of models $\mathrm{MOD}_{\mathrm{Monod}}$ and $\mathrm{MOD}_{\mathrm{Monod}+0 \mathrm{ver}}$ were calculated from those imposed for models MOD $\mathrm{DevMonod}_{\text {and }}$ MOD $\mathrm{D}_{\text {RevMonod+Over }}$ according to Eq. 10 and Eq. 11, respectively. In fact, Moorhead and Sinsabaugh (2006) gave evidence that models based on classical and reverse Monod kinetics would provide similar DECs under the following two constraints:

$$
\begin{aligned}
& k^{\prime} \times C_{B}=k \times C_{S} \\
& \frac{C_{S}}{k_{m}+C_{S}}=\frac{C_{B}}{k_{r m}+C_{B}}
\end{aligned}
$$

Limits for the resistant fraction of soil $\mathrm{C}\left(f C_{Y R}\right.$ soil $)$ were set by assuming that $C_{Y R \text { soil }}$ represents $1-10 \%$ of soil C (Hadas et al., 2004). Microbial biomass $\mathrm{C}$ was initialised at $2 \%$ of soil C (Blagodatskaya and Kuzyakov, 2008; Wutzler and Reichstein, 2013). The microbial biomass $\mathrm{C}$ to $\mathrm{N}$ ratio was let to vary between 4 and 12 (Hassink, 1994; Friedel and Gabiel, 2001; Cleveland and Liptzin, 2007; Griffiths et al., 2012), by changing the $\mathrm{N}$ content of the pool $B$.

The obtained critical $\mathrm{C}$ to $\mathrm{N}$ ratio (Eq. 5) averaged 18 and was in agreement, with values commonly determined experimentally (in the range 15-35) for soils amended with crop residues (Trinsoutrot et al., 2000; Jensen et al., 2005), manure components (Van Kessel et al., 2000), and animal manure and organic fertilisers (Sørensen et al., 2003; Peters and Jensen, 2011; Delin et al., 2012).

The fraction of input organic $\mathrm{N}$ allocated to the pool $Y_{H L}\left(f N_{Y H L}\right)$ was calculated in order to keep the $\mathrm{C}$ to $\mathrm{N}$ ratio of the pool $Y_{H L}$ equal to 15 . This value permitted to explore different partitioning of input $\mathrm{C}$ and $\mathrm{N}$ among the $Y_{L}, Y_{R}$ and $Y_{H L}$ pools, avoiding that the sum of $f N_{Y L}, f N_{Y R}$ and $f N_{Y H L}$ was higher than one, and ensuring that the $\mathrm{C}$ to $\mathrm{N}$ ratio of the pool $Y_{H L}$ was close to that of $O$.

\section{Variability of model outputs}

Besides reporting sensitivity indices, we decided to show also the variation of model outputs over time. For each model output $\left(\mathrm{CO}_{2}\right.$ or SMN, at one of the seven dates) we selected four reference points cor- 
A

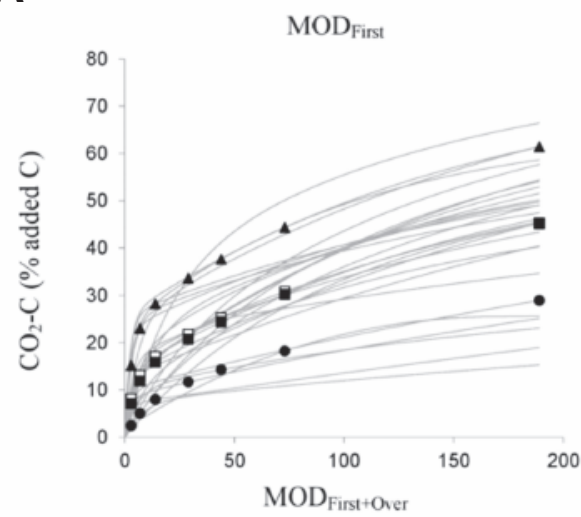

口 Average - Median - 10th percentile A 90th percentile - Simulations
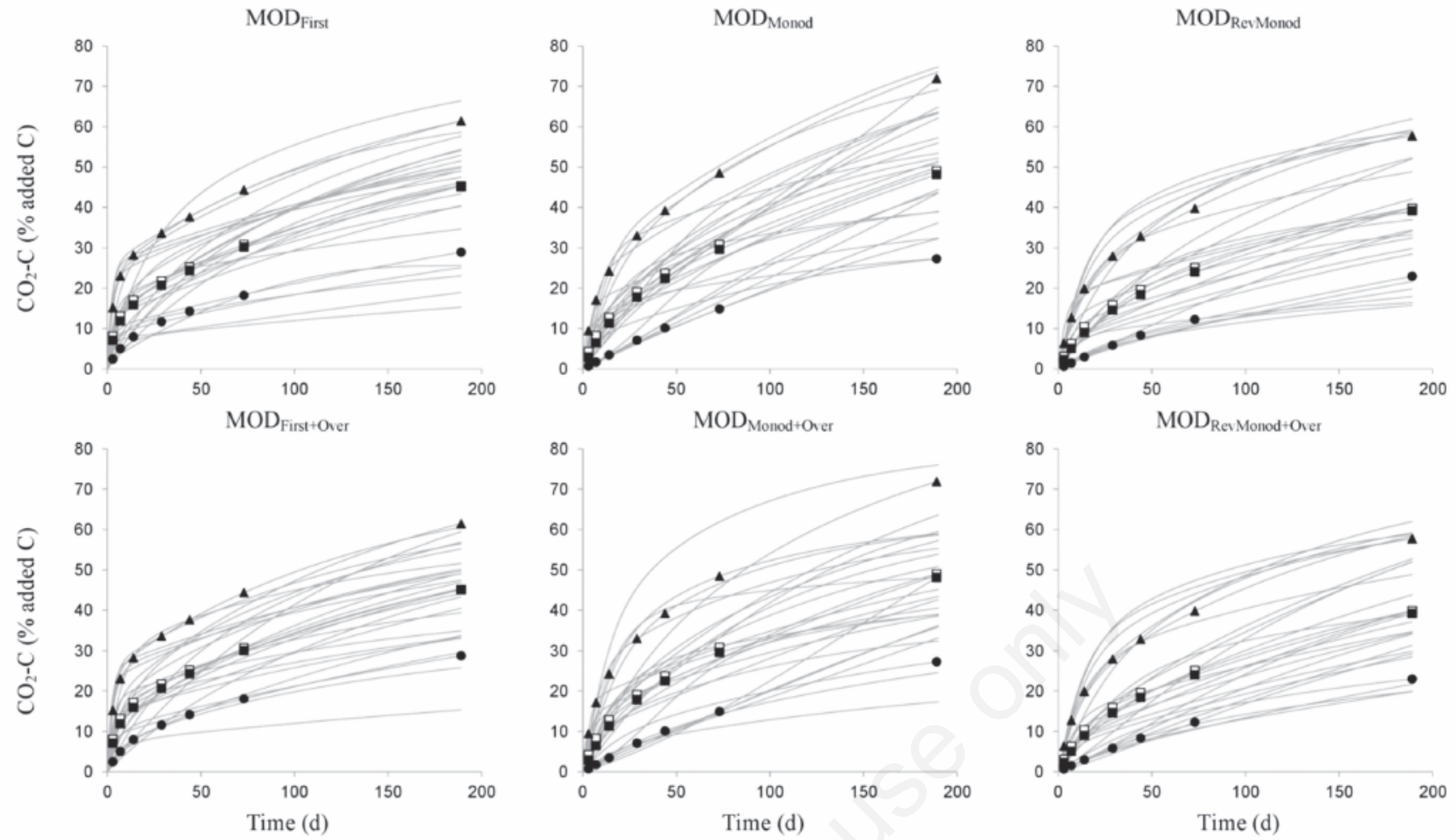

B
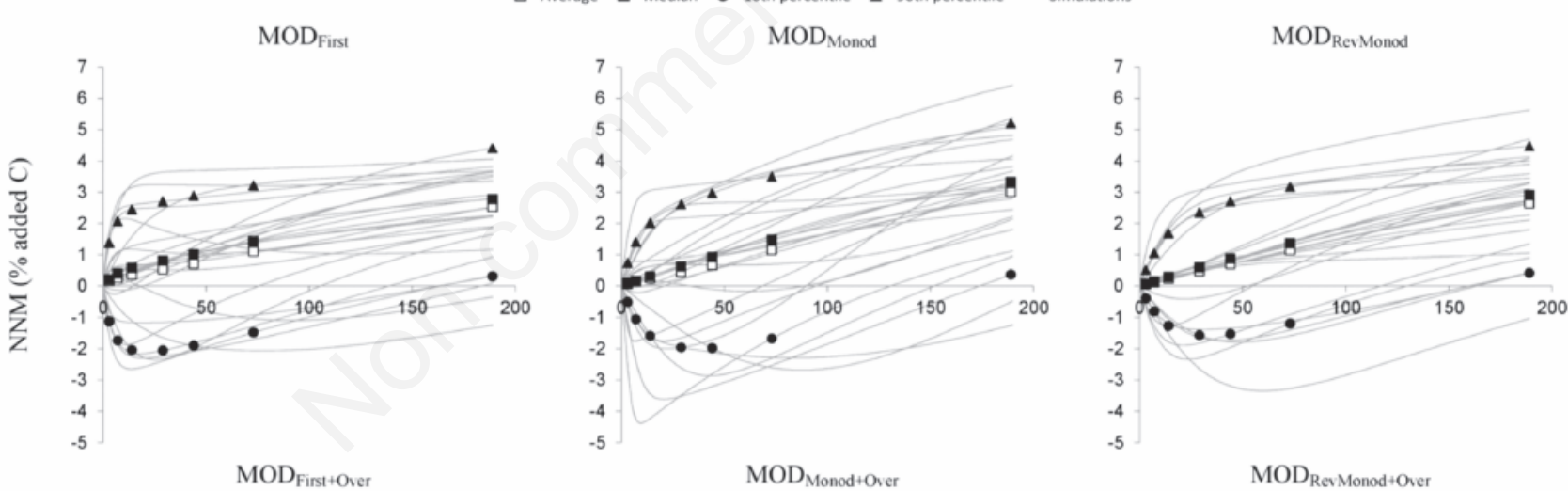

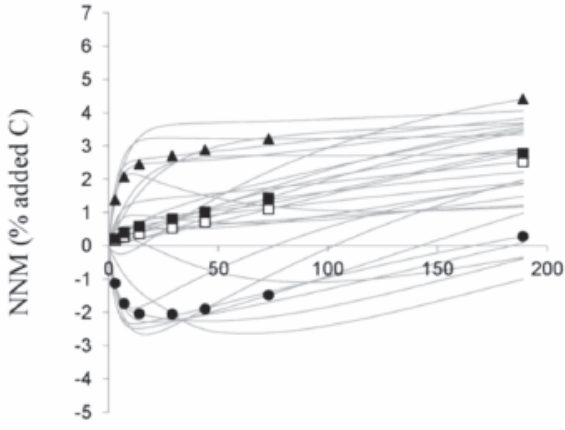

Time (d)

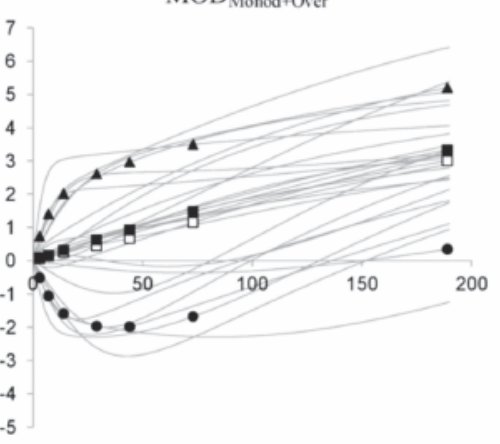

Time (d)

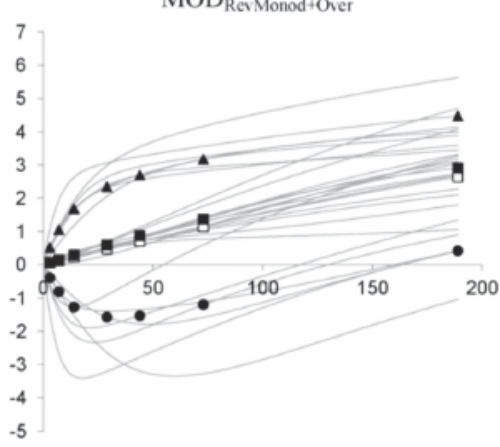

Time (d)

Figure 2. A) Net $\mathrm{CO}_{2}$ emissions and $\mathrm{B}$ ) net nitrogen mineralisation (NNM) following incorporation in the soil of liquid manure, simulated with the six models. Grey lines represent a sample of 28 simulations chosen to represent the variability of the larger set of simulations obtained with sensitivity analysis. Symbols represent the statistical distribution of the simulated variables in seven dates. 
responding to the average, median, $10^{\text {th }}$ and $90^{\text {th }}$ percentile of the distribution of model output obtained after SA. Four reference points by seven dates yielded 28 model simulations. These 28 model simulations (different for $\mathrm{CO}_{2}$ and for $\mathrm{SMN}$ ), for each treatment and model, were selected as representative of the variation in model outputs, and thus plotted for the entire incubation period.

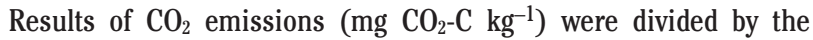
amount of $\mathrm{C}$ applied with manures or crop residue $\left(\mathrm{mg} \mathrm{C} \mathrm{kg}^{-1}\right)$, thus yielding the percentage of applied $\mathrm{C}$ that was mineralized $\left(\mathrm{mg} \mathrm{CO}_{2}-\mathrm{C}\right.$ $100 \mathrm{mg}^{-1}$ of applied C). Results of net SMN (mg N kg-1) are presented as net $\mathrm{N}$ mineralisation (NNM), expressed as percentage of applied $\mathrm{C}$. The NNM (mg N $100 \mathrm{mg}^{-1}$ of applied C) represents the variation of net SMN compared to Day 0 (i.e., $\mathrm{NNM}_{\text {Day }=\mathrm{i}}=\left(\mathrm{SMN}_{\text {Day }=\mathrm{i}}-\mathrm{SMN}_{\text {Day } 00}\right) /$ applied C). Thus, an increase of NNM indicates net mineralisation of organic $\mathrm{N}$, while a decrease of NNM indicates net immobilisation of SMN. We have chosen to standardise NNM per unit of added $\mathrm{C}$ in order to be consistent with a way many experimental measurements of NNM are reported.

\section{Results}

\section{Variability of $\mathrm{CO}_{2}$ emissions and soil mineral nitrogen}

The trend over time and the variability of simulated net $\mathrm{CO}_{2}$ and NNM obtained during SAs is reported in Figures 2-4.

\section{Liquid manure amended soil}

In the LM treatment, the implementation of the C-overflow mechanism produced no appreciable differences in net $\mathrm{CO}_{2}$ (Figure 2A) and NNM (Figure 2B). Conversely, the replacement of first-order with Monod kinetics slightly reduced the amount of mineralised C (4-8\% of manure-C) in the first two weeks (Figure 2A). As incubation proceeded, net $\mathrm{CO}_{2}$ emissions raised slightly faster in $\mathrm{MOD}_{\text {Monod }}$ and MOD $_{\text {Monod+Over }}$ than in other models. At Day 189 , less $\mathrm{C}$ was mineralised in models with first-order and reverse Monod kinetics than in models with classical Monod kinetics (on average $6 \%$ and $11 \%$ of manure-C, respectively) in all simulations with the exception of those corresponding to $10^{\text {th }}$ percentile of $\mathrm{CO}_{2}$ distributions. In such case, $\mathrm{MOD}_{\text {First }}$ and MOD $_{\text {Monod }}$ decomposed the same amount of manure-C (on average $28 \%$ ), and slightly more than $\mathrm{MOD}_{\text {RevMonod }}(23 \%$ of manure-C).

Until Day 14, with Monod kinetics respect to first-order kinetics NNM was higher (by $0.5-0.8 \%$ of manure-C) in simulations immobilising $\mathrm{N}$ (those identified with the $10^{\text {th }}$ percentile points in Figure $2 \mathrm{~B}$ ), or lower (by $0.1-0.8 \%$ of manure $\mathrm{C}$ ) in simulations mineralising $\mathrm{N}$ (average, median and $90^{\text {th }}$ percentile points). Thereafter, models with firstorder and reverse Monod kinetics gave similar NNMs, while those implementing classical Monod equations mineralised more $\mathrm{N}$ (0.5$0.8 \%$ of manure-C at Day 189) in simulations corresponding to average, median and $90^{\text {th }}$ percentile points, while no differences among models occurred in simulations corresponding to the $10^{\text {th }}$ percentile points (Figure 2B).

\section{Solid manure amended soil}

Opposite of LM, replacing the $\mathrm{N}$ inhibition hypothesis with the $\mathrm{C}$ overflow mechanism slightly enhanced manure- $\mathrm{C}$ and $\mathrm{N}$ mineralisation in SM (Figure 3). This occurred especially for models implementing first-order and classical Monod decomposition kinetics, with the exceptions of simulations corresponding to the $90^{\text {th }}$ percentile of output distributions (Figure 3A and B). At the end of incubation (Day 189), extra

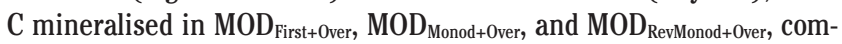
pared to respective model variants implementing the $\mathrm{N}$ inhibition hypothesis ( $\mathrm{MOD}_{\text {First }}, \mathrm{MOD}_{\text {Monod }}$, and $\mathrm{MOD}_{\text {RevMonod }}$ ), corresponded to 3$12 \%, 3-8 \%$ and $2-5 \%$ of manure-C, respectively (Figure $3 \mathrm{~A}$ ). Similarly, results of NNM (Figure 3B) showed that extra $\mathrm{N}$ mineralised in models implementing the $\mathrm{C}$-overflow mechanism increased as net $\mathrm{N}$ immobilisation rose: from $0.1-0.4 \%$ of manure-C at Day 189 for simulations corresponding to average and median, up to $0.5-0.8 \%$ of manure-C for simulations corresponding to $10^{\text {th }}$ percentile of NNM distribution.

Replacing first-order with Monod decomposition kinetics produced different results over time for both net $\mathrm{CO}_{2}$ emissions and NNM. Until Day 14 (Figure $3 \mathrm{~A}$ ), mineralised $\mathrm{C}$ in $\mathrm{MOD}_{\text {First }}$ and $\mathrm{MOD}_{\text {First+0ver }}$ was slightly higher compared to that of models with Monod (by 2-5\% of manure-C) and reverse Monod kinetics (by 2-7\% of manure-C). After Day 14, as previously observed for $\mathrm{LM}, \mathrm{CO}_{2}$ emissions occurred at faster rates in $\mathrm{MOD}_{\text {Monod }}$ and $\mathrm{MOD}_{\text {Monod+Over }}$ than in other models, and at Day 189 , net $\mathrm{CO}_{2}$ was higher, on average by 5 and $7 \%$ of manure-C, compared to models with first-order and reverse Monod kinetics, respectively (Figure 3A).

Differently from net $\mathrm{CO}_{2}$, NNM was higher in models with Monod kinetics than in those with first-order kinetics (by 0.1-1.0\% of manureC), and, in models implementing classical Monod compared to reverse Monod equations (by $0.1-0.4 \%$ of manure-C); moreover, differences among models amplified as incubation proceeded (Figure 3B).

\section{Crop residue amended soil}

In $\mathrm{CR}$, the implementation of $\mathrm{C}$-overflow mechanism always enhanced $C$ mineralisation, even if the effect was narrow $(+1-2 \%$ of residue-C at Day 189) for those corresponding to $90^{\text {th }}$ percentile of $\mathrm{CO}_{2}$ distribution (Figure 4A). Conversely, for other points (average, median and $10^{\text {th }}$ percentile), the increase at Day 189 corresponded to $7-19 \%, 4 \%$ and $2-3 \%$ of residue-C for models implementing first-order, classical, and reverse Monod kinetics, respectively. Conversely, the effect of $\mathrm{C}$ overflow on NNM (Figure 4B) was relevant only for models with firstorder and classical Monod kinetics in simulations corresponding to $10^{\text {th }}$ percentile points after Day 73. In fact, NNM at Day 189 was higher in $\mathrm{MOD}_{\text {First+Over, }} \mathrm{MOD}_{\text {Monod +0ver, }}$ and $\mathrm{MOD}_{\text {RevMonod +Over }}$ (by $0.6 \%, 0.3 \%$, and $0.1 \%$ of residue-C, respectively), compared to respective model variants implementing the $\mathrm{N}$ inhibition hypothesis.

As observed for manured treatments, substituting first-order with Monod decomposition kinetics had an appreciable effect on both net $\mathrm{CO}_{2}$ emissions and NNM also in CR. At the end of incubation, in simulations corresponding to average, median and $90^{\text {th }}$ percentile of net $\mathrm{CO}_{2}$ distribution, more $\mathrm{C}$ was mineralised, corresponding to $1-9 \%$ and 6 $16 \%$ of residue-C, in models with first-order kinetics compared to those with classical and reverse Monod equation, respectively (Figure 4A). The opposite happened in simulations corresponding to $10^{\text {th }}$ percentile of net $\mathrm{CO}_{2}$ distributions, when more $\mathrm{CO}_{2}$ accumulated in both $\mathrm{MOD}_{\text {Monod }}$ and $\mathrm{MOD}_{\text {RevMonod }}$ (on average $10 \%$ of residue-C) compared to $\mathrm{MOD}_{\text {First. }}$. During all the incubation, NNM was higher $(+0.6-1.2 \%$ of residue-C at Day 189) in models with Monod kinetics than in those with first-order kinetics, while differences between models implementing classical and reverse Monod equations were extremely small (very frequently lower than $0.1 \%$ of residue-C).

\section{Sensitivity indices}

After SAs, parameters affecting $\mathrm{C}$ and $\mathrm{N}$ turnover were considered important if they had a first-order sensitivity index higher than $10 \%$ for at least one of the output variables $\left(\mathrm{CO}_{2}\right.$ or $\left.\mathrm{SMN}\right)$ in at least one date. The mean and the standard deviation of first-order sensitivity indices were calculated across the seven dates, separately for each treatment and output variable (Table 5). Moreover, a qualitative trend of the indices over time was given, separately for the short-medium term (Day 3-44) and medium-long term (Day 44-189). The trend was 
A

ㅁ Average - Median $\bullet$ 10th percentile A 90th percentile - Simulations
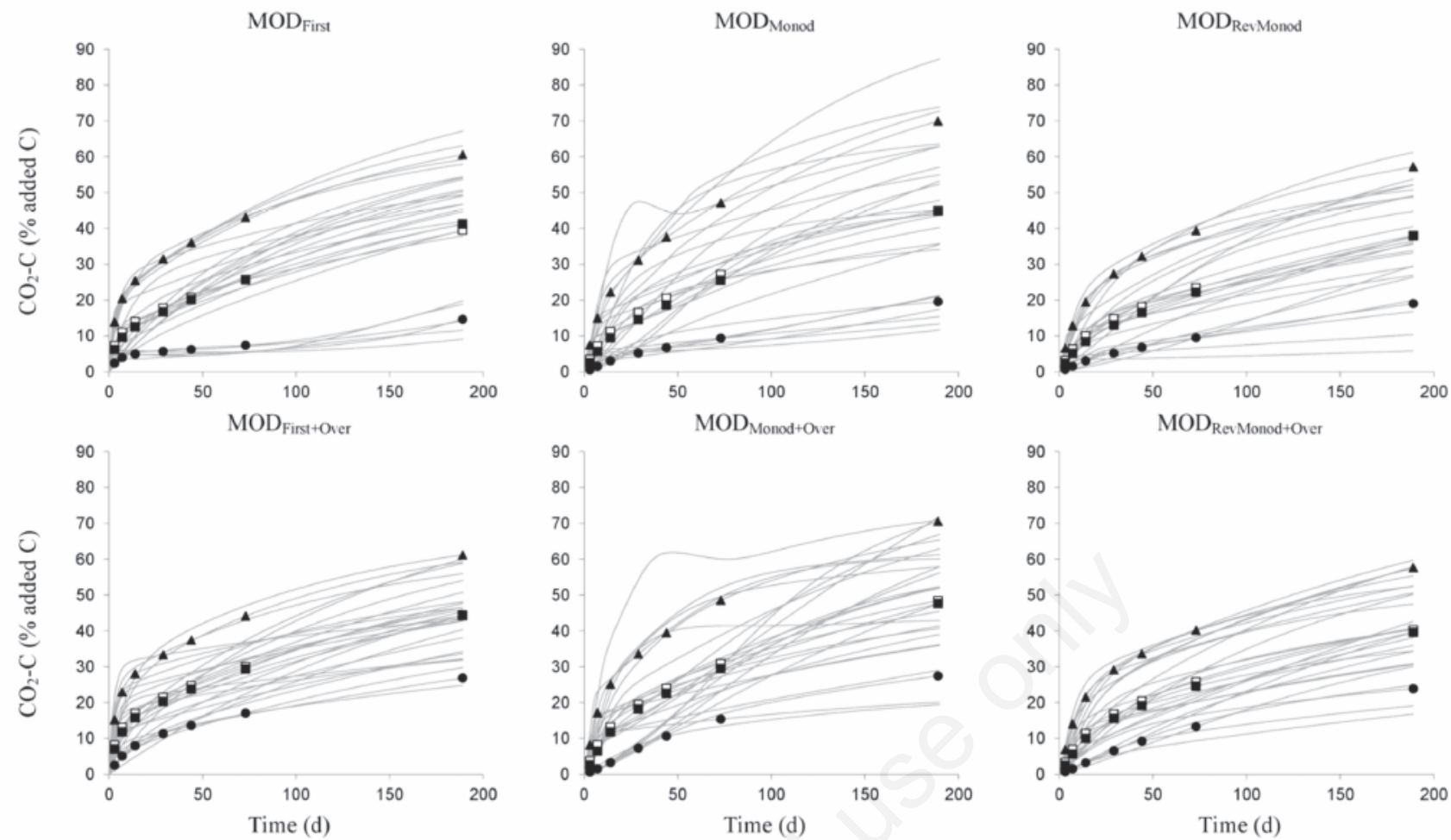

B

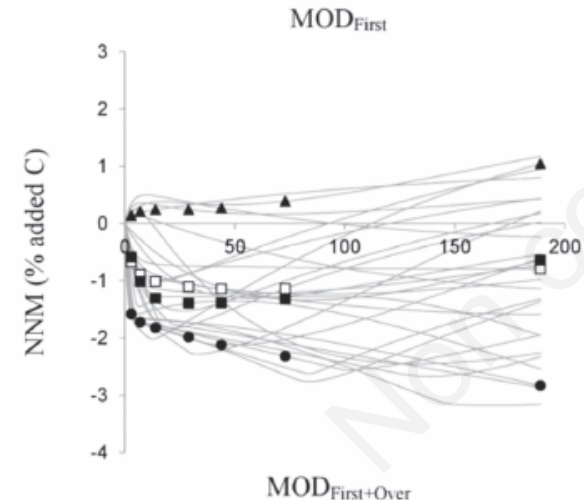

- Average - Median - 10th percentile A 90th percentile — Simulations

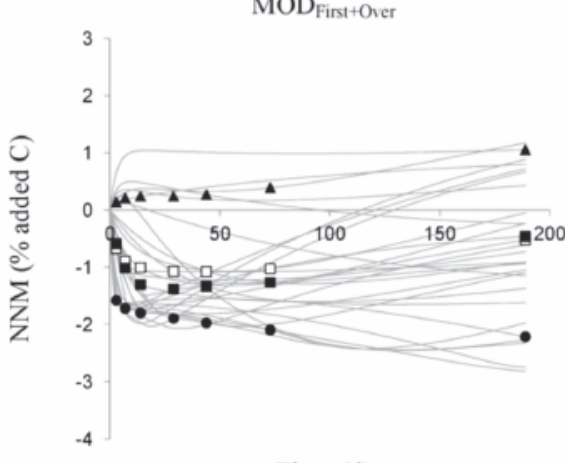

Time (d)

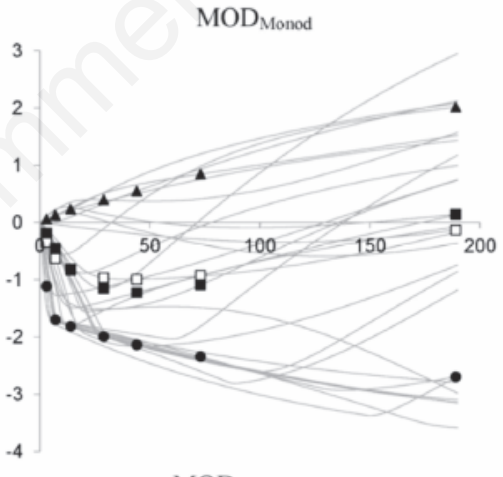

MOD Monod + Over

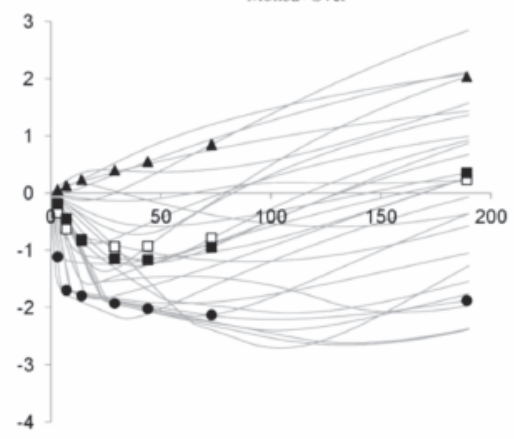

Time (d)

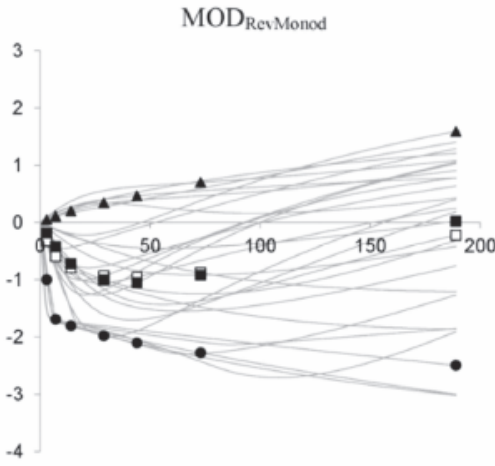

MOD $_{\text {Rei Monod }+ \text { Over }}$

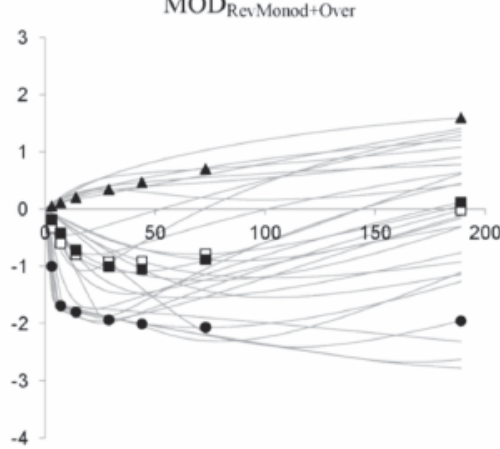

Time (d)

Figure 3. A) Net $\mathrm{CO}_{2}$ emissions and $\mathrm{B}$ ) net nitrogen mineralisation (NNM) following incorporation in the soil of solid manure, simulated with the six models. Grey lines represent a sample of 28 simulations chosen to represent the variability of the larger set of simulations obtained with sensitivity analysis. Symbols represent the statistical distribution of the simulated variables in seven dates. 
assigned three categorical values: increasing, decreasing, and constant. A value, within each time period, was considered constant if its absolute variation $(\max -\min )$ was less than $5 \%$.

\section{Unamended soil}

In $\mathrm{CON}$, parameters that mostly affected $\mathrm{CO}_{2}$ emissions were those related to the turnover of the young resistant pool ( $f C_{Y R}$ soil and $k_{Y R}$ soil) and the substrate use efficiency (eff); these three parameters account together for a relevant fraction of $\mathrm{CO}_{2}$ variance (from 45 to $82 \%$, depending on the model). In addition, in models implementing reverse Monod kinetics, the decomposition constant of microbial biomass $\left(k_{B}\right)$ and the half-saturation constant $\left(k_{r m}\right)$ accounted for a further $29 \%$ of $\mathrm{CO}_{2}$ variance, while in those implementing classical Monod kinetics, the half-saturation constant $\left(k_{m Y R}\right.$ soil $)$ alone explained $26 \%$ of $\mathrm{CO}_{2}$ variation. The importance of microbial biomass parameters eff and $k_{B}$ usually decreased with time, especially after Day 44 , while that of the half-saturation constants increased. Conversely, the effect of $f C_{Y R}$ soil increased after Day 44 in all models, while the trend of $k_{Y R}$ soil depended on the type of decomposition kinetics: $S_{i}$ decreased after Day 44 in

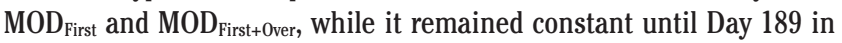
all other models.

The three microbial biomass parameters eff, $f N_{B}$ and, with the exception of $\mathrm{MOD}_{\text {Monod }}$ and $\mathrm{MOD}_{\text {Monod }+ \text { ver }}, k_{B}$, explained a relevant fraction also of the variation of SMN (from 56 to $69 \%$ depending on the model). As observed for $\mathrm{CO}_{2}$, first-order indices of these parameters usually tended to decrease after Day 44.

\section{Amended soil}

As expected, in the amended treatments parameters related to the turnover of pools $C_{Y R}$ soil and $O$ had no appreciable effect in any model (first-order indices always less than 10\%) on both net $\mathrm{CO}_{2}$ emissions and net SMN. Microbial biomass parameters had an effect similar to what was observed in CON. In addition, in the amended treatments some input-related parameters markedly contributed to net $\mathrm{CO}_{2}$ and net SMN variances. With few exceptions, average first-order indices of the labile fraction of input $\mathrm{C}\left(f C_{Y L}\right)$ were high for both net $\mathrm{CO}_{2}(5-25 \%)$ and net SMN (9-23\%) in all models. Moreover, the importance of $f C_{Y L}$ usually diminished as time passed, and as the $\mathrm{C}$ to $\mathrm{N}$ ratio of the input raised (especially from SM to $\mathrm{CR}$ ).

Other input parameters that had an effect on net $\mathrm{CO}_{2}$ variation were the decomposition constants of $Y_{L}$ and $Y_{R}$ pools ( $k_{Y L}$ and $k_{Y R}$ ) that accounted together for $9-40 \%$ of net $\mathrm{CO}_{2}$ variance. Similarly to $f C_{Y L}$, the effect of first-order indices of $k_{Y L}$ decreased with increasing the $\mathrm{C}$ to $\mathrm{N}$ ratio of the input; the opposite happened for $k_{Y R}$, especially for manures and CR, with few exceptions. Also the trend over time of $S_{i}$ differed between $k_{Y L}$ and $k_{Y R}$ : the former had a decreasing effect, while the latter had an increasing effect over time. Differently from what was observed for net $\mathrm{CO}_{2}$, sensitivity of net $\mathrm{SMN}$ to $k_{Y L}$ was relevant in SM (for models implementing classical and reverse Monod kinetics), while sensitivity to $k_{Y R}$ was relevant in CR (for all models). The effect of $k_{Y R}$ usually increased until Day 44 and then stabilised thereafter.

In models with Monod kinetics, also half-saturation constants were important $\left(k_{r m}, k_{m Y L}\right.$ and, with the exception of SM, $\left.k_{m Y R}\right)$. These parameters gave an average contribution of $8-25 \%$ to net $\mathrm{CO}_{2}$ variance and, solely for CR, of $2-8 \%$ to net SMN variance. For both output variables, sensitivity indices of $k_{r m}$ and $k_{m Y L}$ decreased until Day 44, and then remained constant until Day 189, while those of $k_{m Y R}$ usually increased during time.

\section{Parameter interactions}

The sum of first-order sensitivity indices $\left(\Sigma S_{i}\right)$ accounted for $70-94 \%$ and $57-88 \%$ of total output variance for $\mathrm{CO}_{2}$ and SMN, respectively
(Table 5), showing that interactions among model parameters $\left(1-\Sigma S_{i}\right)$ considerably contributed to total output variance in some model $\times$ input combinations, while in other combinations the interactions were not important. Moreover, interactions among parameters increased with increasing the $\mathrm{C}$ to $\mathrm{N}$ ratio of the input, especially in models implementing the $\mathrm{N}$ inhibition hypothesis (Table 5).

In most cases, parameters with high interactions (Table 6) were the same that had high first-order indices. In CON the most important interactions affecting $\mathrm{CO}_{2}$ variance were for parameters $f C_{Y R}$ soil, $k_{Y R}$ soil and the half-saturation constant $k m_{Y R}$ soil. Interactions of microbial biomass parameters eff and $f N_{B}$ also markedly contributed to $S M N$ variance. Besides eff and $f N_{B}$, in the amended treatments net $\mathrm{CO}_{2}$ and net SMN were also sensitive to interactions of input parameters with high $S_{i}\left(f C_{Y L}, k_{Y L}, k_{Y R}, k_{r m}, k_{m Y L}\right.$, and $\left.k_{m Y R}\right)$ in many model $\times$ input combinations.

\section{Discussion}

In this section, we first compare the simulated dynamics of $\mathrm{CO}_{2}$ emissions and NNM with published experiments. Even if we did not calibrate the six models to reproduce a specific set of measurements, it is important to verify if the simulated statistical distributions and the trends over time are in agreement with real data. Thereafter, we discuss the parameters to which model outputs are most sensitive. We then compare models with respect to $\mathrm{N}$ limitation effects on $\mathrm{C}$ decomposition, and with respect to decomposition kinetics. Finally, we emphasize that some models were able to simulate priming effects.

\section{Variability of simulated $\mathrm{CO}_{2}$ emissions and net nitro- gen mineralisation}

Dynamics of simulated net $\mathrm{CO}_{2}$ emissions in amended treatments (Figures 2A, 3A, and 4A) were in general agreement with those observed in aerobic incubations experiments of soil amended with liquid animal manures (Sørensen and Fernández, 2003; Sørensen et al., 2003; Morvan et al., 2006; Bechini and Marino, 2009; Cavalli et al., 2014), solid animal manures (Thomsen and Olesen, 2000; Calderón et al., 2005; Morvan et al., 2006; Peters and Jensen, 2011) and low-N crop residues (Henriksen and Breland, 1999; Trinsoutrot et al., 2000; Nicolardot et al., 2001; Hadas et al., 2004; Jensen et al., 2005; Redin et al., 2014).

Carbon mineralisation dynamics in SL, SM and CR were described by two phases of progressively decreasing decomposition rates. The first phase, lasting for about two months, was characterised by high and exponentially decreasing $\mathrm{CO}_{2}$ emissions, and was accompanied by the complete depletion of the more labile input organic matter in 14-73 days $\left(Y_{L}\right)$; thereafter, decomposition of the more resistant input organic matter $\left(Y_{R}\right)$ mostly contributed total $\mathrm{CO}_{2}$ emission (Figures $2 \mathrm{~A}, 3 \mathrm{~A}$ and $4 \mathrm{~A})$. During the whole incubation, only a fraction of initial $Y_{R}(36$ $70 \%, 42-67 \%$, and $33-61 \%$ in LM, SM, and CR, respectively) was mineralised (data not shown). Conversely mineralisation of $Y_{H L}$ was insignificant (1-3\% of input C) for all inputs.

In our simulations, the application of exogenous organic matter to soil often induced temporary net immobilisation of $\mathrm{N}$ (up to several weeks) by soil microbial biomass, especially when the $\mathrm{C}$ to $\mathrm{N}$ ratio of the added organic input was far higher than that of the soil microbial biomass, as demonstrated experimentally for solid manures (Calderón et al., 2005; Morvan et al., 2006; Peters and Jensen, 2011) and low-N crop residues (Henriksen and Breland, 1999; Trinsoutrot $e t$ al., 2000; Nicolardot et al., 2001; Hadas et al., 2004; Jensen et al., 2005; Redin et al., 2014). This pattern was well represented by all 
A

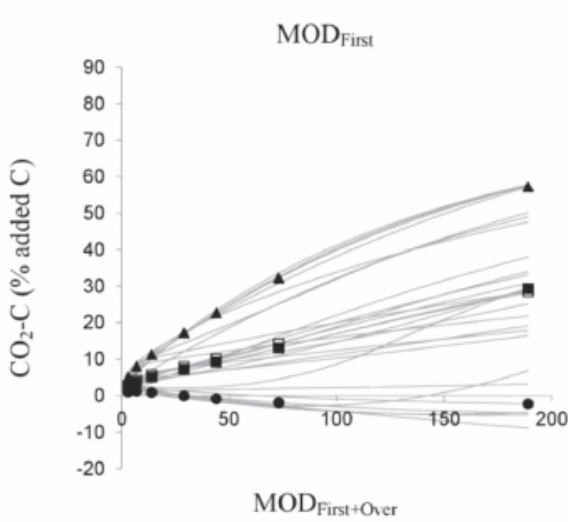

Average - Median - 10th percentile \ 90th percentile - Simulations

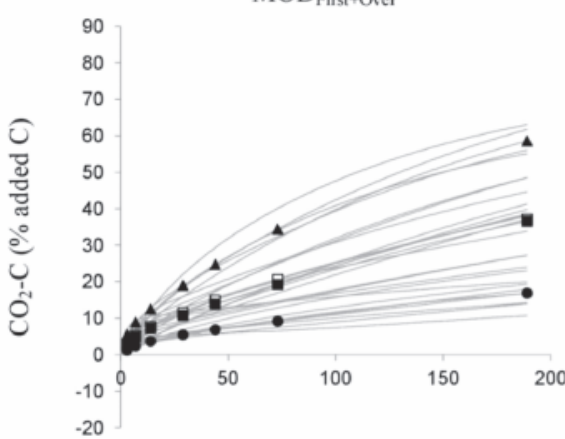

Time (d)
MODMonod

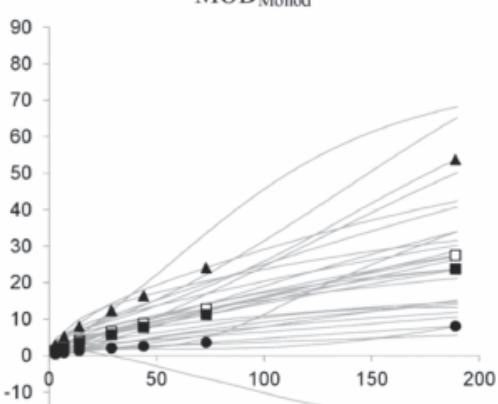

MOD ${ }_{\text {Monod+Over }}$

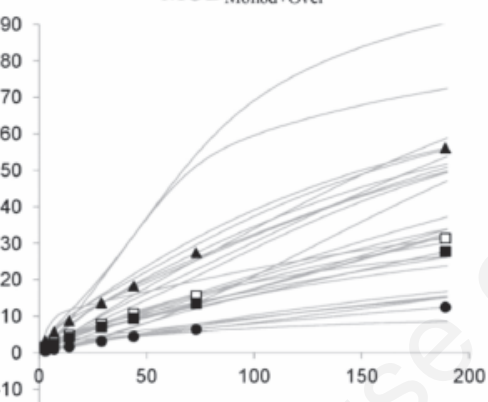

Time (d)
MODReiMonod
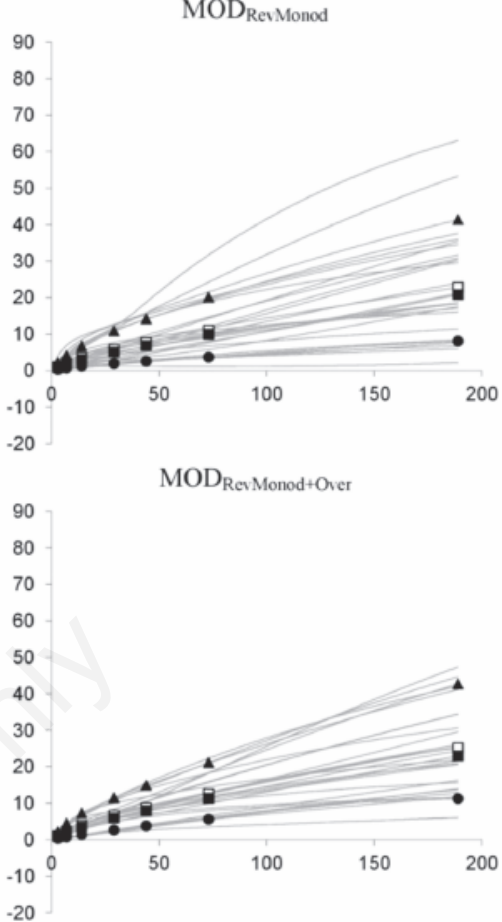

Time (d)

B

․ Average - Median - 10th percentile $\Delta$ 90th percentile - Simulations
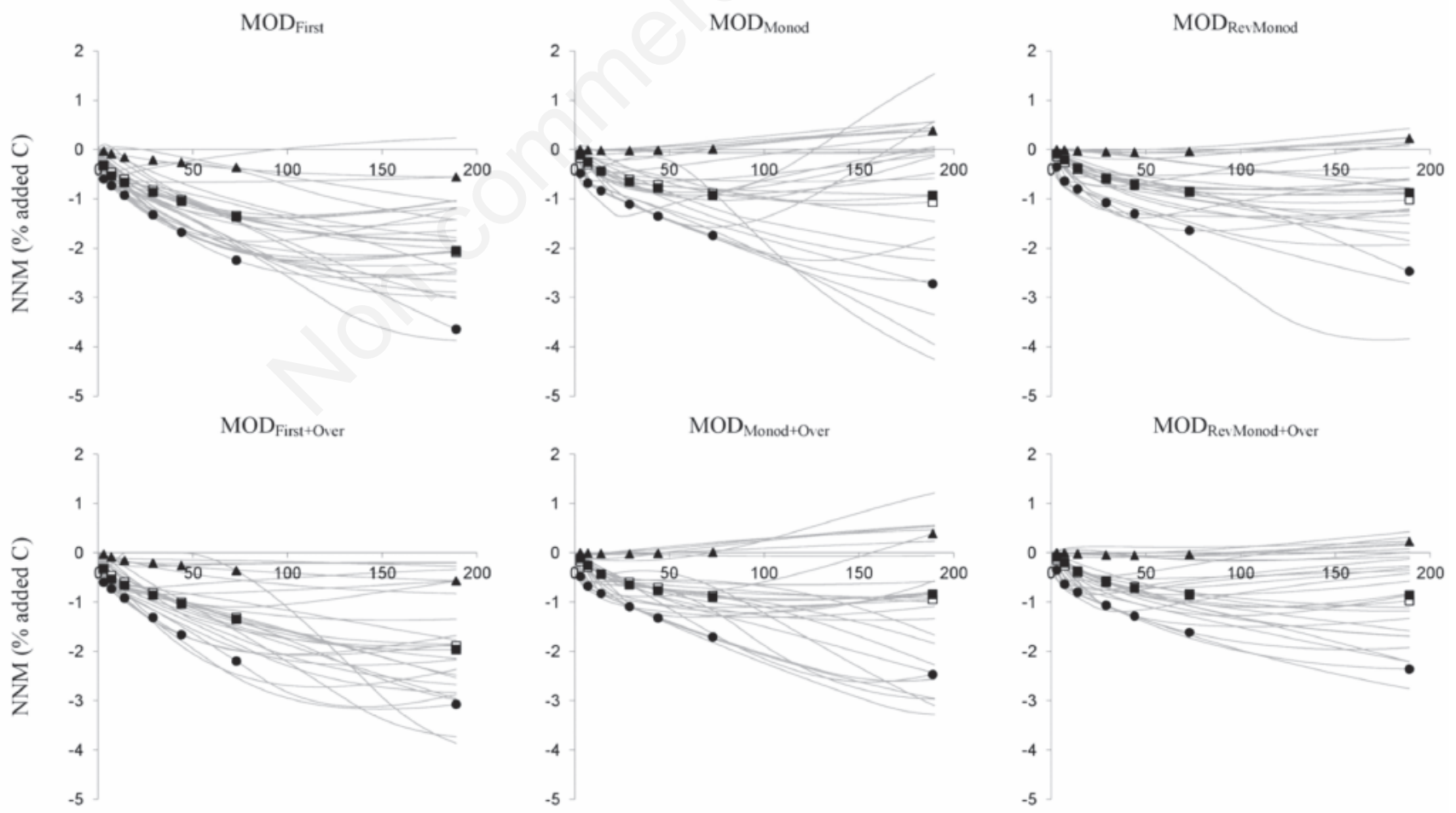

Time (d)

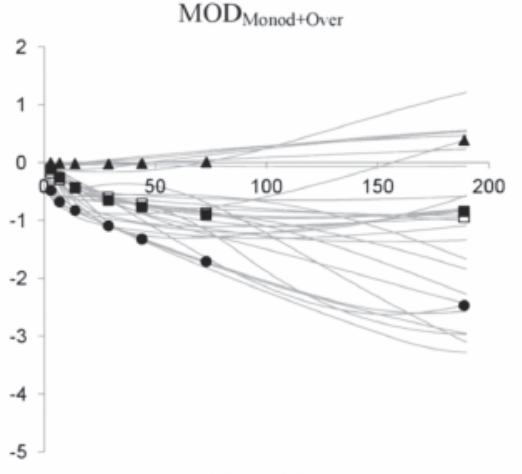

Time (d)

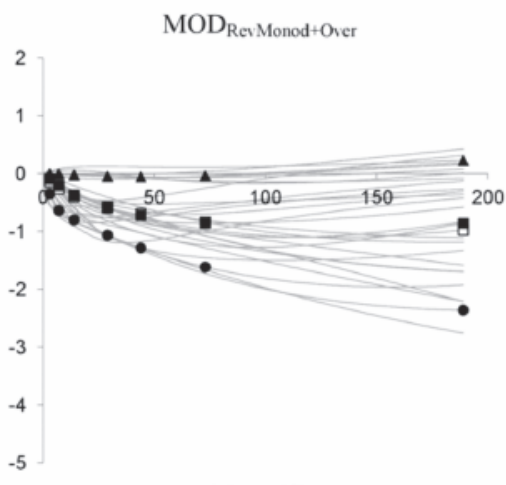

Time (d)

Figure 4. A) Net $\mathrm{CO}_{2}$ emissions and $\mathrm{B}$ ) net nitrogen mineralisation (NNM) following incorporation in the soil of low-N crop residue, simulated with the six models. Grey lines represent a sample of 28 simulations chosen to represent the variability of the larger set of simulations obtained with sensitivity analysis. Symbols represent the statistical distribution of the simulated variables in seven dates. 
Table 5. Average Sobol' first-order sensitivity indices $(\mathrm{Si}, \%)$ calculated in seven dates over the period Day 3-189 ( \pm standard deviation).

\begin{tabular}{|c|c|c|c|c|c|c|c|c|c|}
\hline \multirow[t]{3}{*}{ Model } & \multirow[t]{3}{*}{ Parameter* } & \multicolumn{6}{|c|}{ Input } & \multirow{2}{*}{\multicolumn{2}{|c|}{ Low-N crop residue (CR) }} \\
\hline & & \multicolumn{2}{|c|}{ None (CON) } & \multicolumn{2}{|c|}{ Liquid manure (LM) } & \multicolumn{2}{|c|}{ Solid manure (SM) } & & \\
\hline & & $\mathrm{CO}_{2}$ & SMN & Net $\mathrm{CO}_{2}$ & Net SMN & Net $\mathrm{CO}_{2}$ & Net SMN & Net $\mathrm{CO}_{2}$ & Net SMN \\
\hline $\mathrm{MOD}_{\text {First }}$ & $\begin{array}{l}f C_{Y R} \text { soil } \\
k_{Y R} \text { soil } \\
k_{0} \\
f N_{B} \\
e f f \\
f_{B} \\
k_{B} \\
f C_{Y L} \\
f C_{Y H L} \\
f N_{Y L} \\
k_{Y L} \\
k_{Y R} \\
k_{Y H L} \\
\Sigma S_{i}\end{array}$ & $\begin{array}{c}25 \pm 3(\approx \uparrow) \\
28 \pm 4(\approx \downarrow) \\
2 \pm 2 \\
0 \pm 0 \\
29 \pm 1(\approx \approx) \\
1 \pm 1 \\
3 \pm 0 \\
- \\
- \\
- \\
- \\
- \\
- \\
89 \pm 1\end{array}$ & $\begin{array}{c}3 \pm 4(\approx \uparrow) \\
2 \pm 3 \\
0 \pm 1 \\
10 \pm 5(\downarrow \downarrow) \\
50 \pm 3(\uparrow \downarrow) \\
0 \pm 0 \\
9 \pm 2(\approx \approx) \\
- \\
- \\
- \\
- \\
- \\
- \\
74 \pm 6\end{array}$ & $\begin{array}{c}0 \pm 0 \\
0 \pm 0 \\
0 \pm 0 \\
0 \pm 0 \\
41 \pm 12(\uparrow \approx) \\
1 \pm 2 \\
1 \pm 1 \\
24 \pm 9(\uparrow \downarrow) \\
0 \pm 1 \\
0 \pm 0 \\
16 \pm 17(\downarrow \approx) \\
9 \pm 10(\uparrow \uparrow) \\
0 \pm 0 \\
93 \pm 2\end{array}$ & $\begin{array}{c}0 \pm 0 \\
0 \pm 0 \\
0 \pm 0 \\
35 \pm 7(\uparrow \downarrow) \\
28 \pm 15(\uparrow \uparrow) \\
0 \pm 0 \\
2 \pm 4(\approx \uparrow) \\
20 \pm 12(\downarrow \downarrow) \\
0 \pm 0 \\
2 \pm 1 \\
1 \pm 0 \\
0 \pm 0 \\
0 \pm 0 \\
88 \pm 4\end{array}$ & $\begin{array}{c}0 \pm 0 \\
0 \pm 0 \\
0 \pm 0 \\
8 \pm 3(\uparrow \approx) \\
54 \pm 12(\uparrow \approx) \\
0 \pm 1 \\
2 \pm 2 \\
5 \pm 4(\downarrow \approx) \\
0 \pm 0 \\
0 \pm 0 \\
10 \pm 13(\downarrow \approx) \\
3 \pm 3 \\
0 \pm 0 \\
82 \pm 2\end{array}$ & $\begin{array}{c}0 \pm 0 \\
0 \pm 0 \\
0 \pm 0 \\
33 \pm 5(\approx \downarrow) \\
21 \pm 13(\uparrow \uparrow) \\
0 \pm 0 \\
1 \pm 2 \\
21 \pm 12(\downarrow \downarrow) \\
0 \pm 0 \\
1 \pm 0 \\
2 \pm 3 \\
3 \pm 3 \\
0 \pm 0 \\
82 \pm 4\end{array}$ & $\begin{array}{c}0 \pm 0 \\
0 \pm 0 \\
0 \pm 0 \\
13 \pm 5(\uparrow \downarrow) \\
52 \pm 5(\uparrow \approx) \\
0 \pm 0 \\
2 \pm 2 \\
2 \pm 3 \\
0 \pm 0 \\
0 \pm 0 \\
5 \pm 9(\downarrow \approx) \\
4 \pm 2 \\
0 \pm 0 \\
80 \pm 1\end{array}$ & $\begin{array}{c}0 \pm 0 \\
0 \pm 0 \\
0 \pm 0 \\
15 \pm 11(\downarrow \uparrow) \\
9 \pm 5(\approx \uparrow) \\
0 \pm 0 \\
1 \pm 1 \\
9 \pm 10(\downarrow \approx) \\
0 \pm 0 \\
1 \pm 1 \\
1 \pm 2 \\
19 \pm 8(\uparrow \approx) \\
0 \pm 0 \\
57 \pm 15\end{array}$ \\
\hline \multirow{19}{*}{$\mathrm{MOD}_{\text {Monod }}$} & $f C_{Y R}$ soil & $9 \pm 4(\approx \uparrow)$ & $1 \pm 2$ & $1 \pm 2$ & $0 \pm 0$ & $0 \pm 0$ & $0 \pm 0$ & $0 \pm 0$ & $0 \pm 0$ \\
\hline & $k_{Y R}$ soil & $17 \pm 2(\approx \approx)$ & $2 \pm 3$ & $1 \pm 1$ & $0 \pm 0$ & $0 \pm 0$ & $0 \pm 0$ & $0 \pm 0$ & $0 \pm 0$ \\
\hline & $k_{0}$ & $1 \pm 0$ & $0 \pm 0$ & $0 \pm 0$ & $0 \pm 0$ & $0 \pm 0$ & $0 \pm 0$ & $0 \pm 0$ & $0 \pm 0$ \\
\hline & $f N_{B}$ & $0 \pm 0$ & $4 \pm 2$ & $0 \pm 0$ & $30 \pm 7(\uparrow \downarrow)$ & $5 \pm 3$ & $30 \pm 6(\uparrow \downarrow)$ & $4 \pm 2$ & $24 \pm 4(\approx \downarrow)$ \\
\hline & eff & $19 \pm 8(\downarrow \downarrow)$ & $38 \pm 7(\approx \downarrow)$ & $14 \pm 5(\uparrow \downarrow)$ & $21 \pm 11(\uparrow \uparrow)$ & $29 \pm 12(\uparrow \approx)$ & $21 \pm 12(\uparrow \uparrow)$ & $28 \pm 9(\uparrow \downarrow)$ & $12 \pm 7(\approx \uparrow)$ \\
\hline & $f_{B}$ & $3 \pm 1$ & $0 \pm 1$ & $1 \pm 2$ & $0 \pm 0$ & $1 \pm 1$ & $0 \pm 0$ & $0 \pm 0$ & $0 \pm 0$ \\
\hline & $k_{B}$ & $5 \pm 3$ & $18 \pm 5(\approx \downarrow)$ & $0 \pm 0$ & $2 \pm 3$ & $1 \pm 1$ & $1 \pm 2$ & $1 \pm 1$ & $1 \pm 1$ \\
\hline & $f C_{Y L}$ & - & - & $19 \pm 7(\uparrow \downarrow)$ & $16 \pm 9(\downarrow \downarrow)$ & $6 \pm 2$ & $18 \pm 10(\approx \downarrow)$ & $5 \pm 3$ & $13 \pm 9(\downarrow \downarrow)$ \\
\hline & $f C_{Y H L}$ & - & - & $0 \pm 0$ & $0 \pm 0$ & $0 \pm 0$ & $0 \pm 0$ & $0 \pm 0$ & $0 \pm 0$ \\
\hline & $f N_{Y L}$ & - & - & $0 \pm 0$ & $2 \pm 0$ & $0 \pm 0$ & $1 \pm 0$ & $0 \pm 0$ & $1 \pm 1$ \\
\hline & $k_{Y L}$ & - & - & $22 \pm 8(\approx \downarrow)$ & $2 \pm 2$ & $19 \pm 12(\downarrow \downarrow)$ & $5 \pm 5(\downarrow \approx)$ & $10 \pm 8(\downarrow \approx)$ & $3 \pm 3$ \\
\hline & $k_{Y R}$ & - & - & $4 \pm 5(\approx \uparrow)$ & $0 \pm 0$ & $3 \pm 3$ & $2 \pm 1$ & $7 \pm 6(\uparrow \uparrow)$ & $12 \pm 7(\uparrow \approx)$ \\
\hline & $k_{Y H L}$ & - & - & $0 \pm 0$ & $0 \pm 0$ & $0 \pm 0$ & $0 \pm 0$ & $0 \pm 0$ & $0 \pm 0$ \\
\hline & $k_{\text {mYR soil }}$ & $26 \pm 2(\uparrow \approx)$ & $3 \pm 5(\approx \uparrow)$ & $1 \pm 1$ & $0 \pm 0$ & $0 \pm 0$ & $0 \pm 0$ & $0 \pm 0$ & $0 \pm 0$ \\
\hline & $k_{m 0}$ & $1 \pm 1$ & $0 \pm 0$ & $0 \pm 1$ & $0 \pm 0$ & $0 \pm 0$ & $0 \pm 0$ & $0 \pm 0$ & $0 \pm 0$ \\
\hline & $k_{m Y L}$ & - & - & $14 \pm 15(\downarrow \approx)$ & $1 \pm 0$ & $8 \pm 11(\downarrow \approx)$ & $2 \pm 3$ & $9 \pm 13(\downarrow \approx)$ & $2 \pm 4(\downarrow \approx)$ \\
\hline & $k_{m Y R}$ & - & - & $6 \pm 6(\uparrow \uparrow)$ & $0 \pm 0$ & $2 \pm 2$ & $1 \pm 1$ & $5 \pm 3(\approx \approx)$ & $7 \pm 3(\uparrow \approx)$ \\
\hline & $k_{\text {mYHL }}$ & - & - & $0 \pm 0$ & $0 \pm 0$ & $0 \pm 0$ & $0 \pm 0$ & $0 \pm 0$ & $0 \pm 0$ \\
\hline & $\Sigma S_{i}$ & $80 \pm 3$ & $65 \pm 2$ & $85 \pm 3$ & $75 \pm 11$ & $75 \pm 1$ & $80 \pm 4$ & $70 \pm 6$ & $75 \pm 5$ \\
\hline \multirow{12}{*}{$\mathrm{MOD}_{\text {RevMonod }}$} & $f C_{Y R}$ soil & $12 \pm 3(\approx \uparrow)$ & $1 \pm 2$ & $0 \pm 1$ & $0 \pm 0$ & $0 \pm 0$ & $0 \pm 0$ & $0 \pm 0$ & $0 \pm 0$ \\
\hline & $k_{Y R}$ soil & $15 \pm 2(\approx \approx)$ & $1 \pm 2$ & $0 \pm 1$ & $0 \pm 0$ & $0 \pm 0$ & $0 \pm 0$ & $0 \pm 0$ & $0 \pm 0$ \\
\hline & $k_{0}$ & $1 \pm 0$ & $0 \pm 0$ & $0 \pm 0$ & $0 \pm 0$ & $0 \pm 0$ & $0 \pm 0$ & $0 \pm 0$ & $0 \pm 0$ \\
\hline & $f N_{B}$ & $\begin{array}{c}0 \pm 0 \\
0(1)\end{array}$ & $9 \pm 2(\approx \approx)$ & $0 \pm 0$ & $30 \pm 5(\uparrow \downarrow)$ & $3 \pm 2$ & $30 \pm 7(\uparrow \downarrow)$ & $2 \pm 2$ & $26 \pm 5(\uparrow \downarrow)$ \\
\hline & & $\begin{array}{c}26 \pm 9(\downarrow \downarrow) \\
4 \pm 1\end{array}$ & $\begin{array}{c}39 \pm 6(\approx \downarrow) \\
0 \pm 1\end{array}$ & $\begin{array}{c}19 \pm 6(\uparrow \approx) \\
1 \pm 2\end{array}$ & $\begin{array}{c}22 \pm 12(\uparrow \uparrow) \\
0 \pm 0\end{array}$ & $\begin{array}{c}33 \pm 14(\uparrow \approx) \\
1 \pm 1\end{array}$ & $\begin{array}{c}21 \pm 14(\uparrow \uparrow) \\
0 \pm 0\end{array}$ & $\begin{array}{c}30 \pm 9(\uparrow \downarrow) \\
0 \pm 1\end{array}$ & $\begin{array}{c}14 \pm 7(\approx \uparrow) \\
0 \pm 0\end{array}$ \\
\hline & $k_{B}$ & $8 \pm 4(\approx \downarrow)$ & $21 \pm 5(\approx \downarrow)$ & $0 \pm 0$ & $2 \pm 3$ & $1 \pm 1$ & $1 \pm 3$ & $1 \pm 1$ & $1 \pm 1$ \\
\hline & $f C_{Y L}$ & - & & $25 \pm 8(\uparrow \approx)$ & $21 \pm 9(\approx \downarrow)$ & $12 \pm 2(\approx \approx)$ & $23 \pm 10(\approx \downarrow)$ & $7 \pm 4(\downarrow \approx)$ & $14 \pm 8(\downarrow \downarrow)$ \\
\hline & $f C_{Y H L}$ & . & - & $0 \pm 0$ & $0 \pm 0$ & $0 \pm 0$ & $0 \pm 0$ & $0 \pm 0$ & $0 \pm 0$ \\
\hline & $f N_{Y L}$ & - & & $0 \pm 0$ & $3 \pm 0$ & $\begin{array}{l}0 \pm 0 \\
+10\end{array}$ & $\begin{array}{r}1 \pm 0 \\
-5\end{array}$ & $0 \pm 0$ & $2 \pm 1$ \\
\hline & $\begin{array}{l}k_{Y L} \\
k_{Y R}\end{array}$ & - & - & $\begin{array}{c}25 \pm 11(\downarrow \downarrow) \\
3 \pm 4(\approx \uparrow)\end{array}$ & $\begin{array}{l}2 \pm 1 \\
0 \pm 0\end{array}$ & $\begin{array}{c}19 \pm 12(\downarrow \downarrow) \\
2+4\end{array}$ & $\begin{array}{c}5 \pm 5(\downarrow \approx) \\
1+1\end{array}$ & $\begin{array}{c}12 \pm 10(\downarrow \downarrow) \\
8 \pm 7 \uparrow \uparrow)\end{array}$ & $\begin{array}{c}3 \pm 4 \\
12+7(\uparrow \approx)\end{array}$ \\
\hline & $\begin{array}{l}K_{Y R} \\
k_{Y H L}\end{array}$ & - & - & $\begin{array}{c}J \pm 4(\approx) \\
0 \pm 0\end{array}$ & $0 \pm 0$ & $\begin{array}{l}2 \pm 4 \\
0 \pm 0\end{array}$ & $\begin{array}{l}1 \pm 1 \\
0 \pm 0\end{array}$ & $\begin{array}{c}0 \pm r(1) \\
0 \pm 0\end{array}$ & $\begin{array}{c}12 \pm r(1 \approx) \\
0 \pm 0\end{array}$ \\
\hline & $\begin{array}{l}k_{r m} \\
\Sigma S\end{array}$ & $21 \pm 5(\approx \uparrow)$ & $2 \pm 4(\approx \uparrow)$ & $\begin{array}{c}14 \pm 6(\downarrow \\
89+4\end{array}$ & $\begin{array}{c}0 \pm 0 \\
80+10\end{array}$ & $8 \pm 7(\downarrow \approx)$ & $\begin{array}{l}2 \pm 3 \\
85+4\end{array}$ & $14 \pm 8(\downarrow \approx)$ & $8 \pm 3(\downarrow \approx)$ \\
\hline \multirow[t]{15}{*}{ MOD $D_{\text {first+Over }}$} & 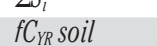 & $25 \pm 3(\approx \uparrow)$ & $3 \pm 5(\approx \uparrow)$ & $0 \pm 0$ & $\begin{array}{c}00 \pm 10 \\
0 \pm 0\end{array}$ & $\begin{array}{l}19 \pm 1 \\
0 \pm 0\end{array}$ & $0 \pm 0$ & $0 \pm 0$ & $\begin{array}{c}19 \pm 0 \\
0 \pm 0\end{array}$ \\
\hline & $\begin{array}{l}\text { MYR soll } \\
k_{Y R} \text { soil }\end{array}$ & $28 \pm 4(\approx \downarrow)$ & $\begin{array}{c} \pm(\approx) \\
2 \pm 3\end{array}$ & $\begin{array}{l}0 \pm 0 \\
0 \pm 0\end{array}$ & $\begin{array}{l}0 \pm 0 \\
0 \pm 0\end{array}$ & $0 \pm 0$ & $\begin{array}{l}0 \pm 0 \\
0 \pm 0\end{array}$ & $\begin{array}{l}0 \pm 0 \\
0 \pm 0\end{array}$ & $\begin{array}{l}0 \pm 0 \\
0 \pm 0\end{array}$ \\
\hline & $k_{0}$ & $2 \pm 2$ & $0 \pm 1$ & $0 \pm 0$ & $0 \pm 0$ & $0 \pm 0$ & $0 \pm 0$ & $0 \pm 0$ & $0 \pm 0$ \\
\hline & $f N_{B}$ & $0 \pm 0$ & $10 \pm 5(\downarrow \downarrow)$ & $0 \pm 0$ & $35 \pm 7(\uparrow \downarrow)$ & $0 \pm 0$ & $32 \pm 7(\approx \downarrow)$ & $1 \pm 1$ & $14 \pm 12(\downarrow \uparrow)$ \\
\hline & eff & $29 \pm 1(\approx \approx)$ & $50 \pm 3(\uparrow \downarrow)$ & $42 \pm 12(\uparrow \approx)$ & $28 \pm 15(\uparrow \uparrow)$ & $45 \pm 15(\uparrow \approx)$ & $23 \pm 16(\uparrow \uparrow)$ & $41 \pm 7(\uparrow \approx)$ & $10 \pm 7(\approx \uparrow)$ \\
\hline & $f_{B}$ & $1 \pm 1$ & $0 \pm 0$ & $1 \pm 2$ & $0 \pm 0$ & $1 \pm 1$ & $0 \pm 1$ & $0 \pm 0$ & $0 \pm 1$ \\
\hline & $k_{B}$ & $3 \pm 0$ & $9 \pm 2(\approx \approx)$ & $1 \pm 2$ & $2 \pm 4(\approx \uparrow)$ & $1 \pm 2$ & $2 \pm 3$ & $1 \pm 1$ & $1 \pm 1$ \\
\hline & $f C_{Y L}$ & - & - & $24 \pm 9(\approx \downarrow)$ & $20 \pm 12(\downarrow \downarrow)$ & $22 \pm 9(\approx \downarrow)$ & $19 \pm 14(\downarrow \downarrow)$ & $10 \pm 9(\downarrow \approx)$ & $9 \pm 10(\downarrow \approx)$ \\
\hline & $f C_{Y H L}$ & - & - & $0 \pm 1$ & $0 \pm 0$ & $0 \pm 1$ & $0 \pm 0$ & $0 \pm 0$ & $0 \pm 0$ \\
\hline & $f N_{Y L}$ & - & - & $0 \pm 0$ & $2 \pm 1$ & $0 \pm 0$ & $1 \pm 0$ & $0 \pm 0$ & $1 \pm 1$ \\
\hline & $k_{Y L}$ & - & - & $16 \pm 17(\downarrow \approx)$ & $1 \pm 0$ & $16 \pm 17(\downarrow \approx)$ & $2 \pm 3$ & $10 \pm 14(\downarrow \approx)$ & $1 \pm 2$ \\
\hline & $k_{Y R}$ & - & - & $9 \pm 10(\uparrow \uparrow)$ & $0 \pm 0$ & $8 \pm 9(\uparrow \uparrow)$ & $4 \pm 4$ & $30 \pm 16(\uparrow \approx)$ & $20 \pm 9(\uparrow \approx)$ \\
\hline & $k_{Y H L}$ & - & - & $0 \pm 0$ & $0 \pm 0$ & $0 \pm 0$ & $0 \pm 0$ & $0 \pm 0$ & $0 \pm 0$ \\
\hline & $k_{P}$ & $0 \pm 0$ & $0 \pm 0$ & $0 \pm 0$ & $0 \pm 0$ & $0 \pm 0$ & $0 \pm 0$ & $0 \pm 0$ & $0 \pm 0$ \\
\hline & $\Sigma S_{i}$ & $89 \pm 2$ & $74 \pm 6$ & $94 \pm 3$ & $88 \pm 4$ & $93 \pm 2$ & $84 \pm 4$ & $93 \pm 2$ & $58 \pm 15$ \\
\hline
\end{tabular}


models after application to soil of both SM (Figure 3B) and CR (Figure 4B).

Conversely, the simulated NNM by all models was excessive, already from the beginning of the incubation, when compared to measurements carried out in incubation studies with LM. In fact, even in the case of liquid manures, with low $\mathrm{C}$ to organic $\mathrm{N}$ ratios, considerable net $\mathrm{N}$ immobilisation is often experimentally observed in the first weeks of manure decomposition (Sørensen and Fernández, 2003; Sørensen et al., 2003), frequently lasting for months (Bechini and Marino, 2009). On the contrary, in our study we observe that only model simulations corresponding to the $10^{\text {th }}$ percentile of net SMN distributions provided manure- $\mathrm{N}$ mineralisation similar to what observed in reality, while the rest of the distributions were far too high (Figure 2B). To explain this fact, we point our attention to the ranges of model parameters used in SAs. These ranges were chosen in order to simulate both $\mathrm{C}$ and $\mathrm{N}$ decomposition. However, Cavalli and Bechini (2012) have shown that, with models characterised by fixed microbial $\mathrm{C}$ to $\mathrm{N}$ ratio and $\mathrm{C}$ use efficiency, a trade- off exists between an accurate simulation of $\mathrm{C}$ and $\mathrm{N}$ mineralisation. In particular, to simulate the immobilisation of $\mathrm{N}$ that is commonly observed in experiments, it is necessary to simulate a very low $\mathrm{C}$ respiration, a situation that, with the ranges of parameter values that we have chosen, occurred rarely.

\section{Parameters common to all models affecting $\mathrm{CO}_{2}$ emis- sions and net nitrogen mineralisation}

Despite the rather high number of parameters needed in the six models (Table 1), respired $\mathrm{C}$ and SMN were sensitive only to a variation of few of them (Table 5) and their interactions (Table 6). In all models, substrate use efficiency ( $e f f$ ) accounted (on average for the entire period) for $14-54 \%$ of $\mathrm{CO}_{2}$ variance. The high sensitivity of $\mathrm{CO}_{2}$ to eff was due to its direct effect on $\mathrm{C}$ turnover (Figure 1), regulating the amount of $\mathrm{C}$ respired as $\mathrm{CO}_{2}$, and its feedback on $\mathrm{C}$ decomposition by defining $\mathrm{N}$ required by microbial biomass (Eq. 4). Thus, when shortage of $\mathrm{N}$ occurs, $\mathrm{CO}_{2}$ emissions are reduced proportionally to the value of $e f f$ by

Table 5. Continued from previous page.

\begin{tabular}{|c|c|c|c|c|c|c|c|c|c|}
\hline \multirow[t]{3}{*}{ Model } & \multirow[t]{3}{*}{ Parameter* } & \multicolumn{8}{|c|}{ Input } \\
\hline & & \multicolumn{2}{|c|}{ None (CON) } & \multicolumn{2}{|c|}{ Liquid manure (LM) } & \multicolumn{2}{|c|}{ Solid manure (SM) } & \multicolumn{2}{|c|}{ Low-N crop residue (CR) } \\
\hline & & $\mathrm{CO}_{2}$ & SMN & Net $\mathrm{CO}_{2}$ & Net SMN & Net $\mathrm{CO}_{2}$ & Net SMN & Net $\mathrm{CO}_{2}$ & Net SMN \\
\hline \multirow{21}{*}{ MOD $_{\text {Monod+Over }}$} & $f C_{Y R}$ soil & $9 \pm 4(\approx \uparrow)$ & $1 \pm 2$ & $1 \pm 2$ & $0 \pm 0$ & $1 \pm 1$ & $0 \pm 0$ & $1 \pm 1$ & $0 \pm 0$ \\
\hline & $k_{Y R}$ soil & $17 \pm 2(\approx \approx)$ & $2 \pm 3$ & $1 \pm 1$ & $0 \pm 0$ & $1 \pm 1$ & $0 \pm 0$ & $1 \pm 1$ & $0 \pm 0$ \\
\hline & $k_{0}$ & $1 \pm 0$ & $0 \pm 0$ & $0 \pm 0$ & $0 \pm 0$ & $0 \pm 0$ & $0 \pm 0$ & $0 \pm 0$ & $0 \pm 0$ \\
\hline & $f N_{B}$ & $0 \pm 0$ & $4 \pm 2$ & $0 \pm 0$ & $30 \pm 7(\uparrow \downarrow)$ & $1 \pm 1$ & $29 \pm 7(\uparrow \downarrow)$ & $1 \pm 1$ & $23 \pm 4(\approx \downarrow)$ \\
\hline & eff & $19 \pm 9(\downarrow \downarrow)$ & $38 \pm 8(\approx \downarrow)$ & $14 \pm 5(\uparrow \downarrow)$ & $21 \pm 11(\uparrow \uparrow)$ & $19 \pm 8(\uparrow \approx)$ & $22 \pm 13(\uparrow \uparrow)$ & $15 \pm 4(\uparrow \downarrow)$ & $12 \pm 8(\approx \uparrow)$ \\
\hline & $f_{B}$ & $3 \pm 1$ & $0 \pm 1$ & $1 \pm 2$ & $0 \pm 0$ & $1 \pm 2$ & $0 \pm 0$ & $0 \pm 1$ & $0 \pm 0$ \\
\hline & $k_{B}$ & $5 \pm 3$ & $18 \pm 5(\approx \downarrow)$ & $0 \pm 0$ & $2 \pm 3$ & $0 \pm 0$ & $2 \pm 4$ & $0 \pm 0$ & $1 \pm 1$ \\
\hline & $f C_{Y L}$ & - & - & $19 \pm 7(\uparrow \downarrow)$ & $16 \pm 9(\downarrow \downarrow)$ & $16 \pm 7(\uparrow \downarrow)$ & $17 \pm 10(\downarrow \downarrow)$ & $10 \pm 5(\approx \downarrow)$ & $12 \pm 9(\downarrow \downarrow)$ \\
\hline & $f C_{Y H L}$ & - & - & $0 \pm 0$ & $0 \pm 0$ & $0 \pm 0$ & $0 \pm 0$ & $0 \pm 0$ & $0 \pm 0$ \\
\hline & $f N_{Y L}$ & - & - & $0 \pm 0$ & $2 \pm 0$ & $0 \pm 0$ & $1 \pm 0$ & $0 \pm 0$ & $1 \pm 1$ \\
\hline & $k_{Y L}$ & - & - & $22 \pm 8(\approx \downarrow)$ & $2 \pm 1$ & $25 \pm 12(\downarrow \downarrow)$ & $5 \pm 5(\downarrow \approx)$ & $12 \pm 9(\downarrow \downarrow)$ & $3 \pm 3$ \\
\hline & $k_{Y R}$ & - & & $4 \pm 5(\approx \uparrow)$ & $0 \pm 0$ & $5 \pm 6(\uparrow \uparrow)$ & $2 \pm 2$ & $15 \pm 12(\uparrow \uparrow)$ & $12 \pm 7(\uparrow \approx)$ \\
\hline & $k_{Y H L}$ & - & 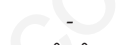 & $0 \pm 0$ & $0 \pm 0$ & $0 \pm 0$ & $0 \pm 0$ & $0 \pm 0$ & $0 \pm 0$ \\
\hline & $k_{P}$ & $0 \pm 0$ & $0 \pm 0$ & $0 \pm 0$ & $0 \pm 0$ & $0 \pm 0$ & $0 \pm 0$ & $0 \pm 0$ & $0 \pm 0$ \\
\hline & $k_{m Y R ~ s o i l}$ & $26 \pm 3(\uparrow \approx)$ & $3 \pm 5(\approx \uparrow)$ & $1 \pm 1$ & $0 \pm 0$ & $0 \pm 0$ & $0 \pm 0$ & $1 \pm 1$ & $0 \pm 0$ \\
\hline & $k_{m 0}$ & $1 \pm 1$ & $0 \pm 0$ & $0 \pm 1$ & $0 \pm 0$ & $0 \pm 1$ & $0 \pm 0$ & $0 \pm 0$ & $0 \pm 0$ \\
\hline & $k_{m Y L}$ & - & - & $14 \pm 15(\downarrow \approx)$ & $1 \pm 0$ & $11 \pm 12(\downarrow \approx)$ & $2 \pm 3$ & $12 \pm 15(\downarrow \approx)$ & $2 \pm 4(\downarrow \approx)$ \\
\hline & $k_{m Y R}$ & & & $-6 \pm 6(\uparrow \uparrow)$ & $0 \pm 0$ & $4 \pm 4$ & $1 \pm 1$ & $15 \pm 10(\uparrow \approx)$ & $7 \pm 3(\uparrow \downarrow)$ \\
\hline & $k_{m Y H L}$ & - & - & $0 \pm 0$ & $0 \pm 0$ & $0 \pm 0$ & $0 \pm 0$ & $0 \pm 0$ & $0 \pm 0$ \\
\hline & $k_{m P}$ & $0 \pm 0$ & $0 \pm 0$ & $0 \pm 0$ & $0 \pm 0$ & $0 \pm 0$ & $0 \pm 0$ & $0 \pm 0$ & $0 \pm 0$ \\
\hline & $\Sigma S_{i}$ & $80 \pm 3$ & $66 \pm 3$ & $85 \pm 3$ & $75 \pm 11$ & $84 \pm 3$ & $81 \pm 4$ & $82 \pm 2$ & $75 \pm 4$ \\
\hline \multirow[t]{16}{*}{ MOD $D_{\text {RevMonod+Over }}$} & $f C_{Y R}$ soil & $12 \pm 3(\approx \uparrow)$ & $1 \pm 2$ & $0 \pm 1$ & $0 \pm 0$ & $0 \pm 0$ & $0 \pm 0$ & $0 \pm 0$ & $0 \pm 0$ \\
\hline & $k_{Y R}$ soil & $15 \pm 2(\approx \approx)$ & $1 \pm 2$ & $0 \pm 1$ & $0 \pm 0$ & $0 \pm 0$ & $0 \pm 0$ & $0 \pm 0$ & $0 \pm 0$ \\
\hline & $k_{0}$ & $1 \pm 0$ & $0 \pm 0$ & $0 \pm 0$ & $0 \pm 0$ & $0 \pm 0$ & $0 \pm 0$ & $0 \pm 0$ & $0 \pm 0$ \\
\hline & $f N_{B}$ & $0 \pm 0$ & $9 \pm 2(\approx \approx)$ & $0 \pm 0$ & $30 \pm 5(\uparrow \downarrow)$ & $0 \pm 0$ & $29 \pm 7(\uparrow \downarrow)$ & $0 \pm 0$ & $25 \pm 5(\approx \downarrow)$ \\
\hline & eff & $26 \pm 9(\downarrow \downarrow)$ & $39 \pm 6(\approx \downarrow)$ & $19 \pm 6(\uparrow \approx)$ & $22 \pm 12(\uparrow \uparrow)$ & $24 \pm 11(\uparrow \approx)$ & $23 \pm 15(\uparrow \uparrow)$ & $19 \pm 5(\uparrow \downarrow)$ & $14 \pm 8(\approx \uparrow)$ \\
\hline & $f_{B}$ & $4 \pm 1$ & $0 \pm 1$ & $1 \pm 2$ & $0 \pm 0$ & $1 \pm 2$ & $0 \pm 0$ & $0 \pm 1$ & $0 \pm 0$ \\
\hline & $k_{B}$ & $8 \pm 4(\approx \downarrow)$ & $21 \pm 5(\approx \downarrow)$ & $0 \pm 0$ & $2 \pm 3$ & $1 \pm 1$ & $2 \pm 4$ & $0 \pm 0$ & $1 \pm 2$ \\
\hline & $f C_{Y L}$ & - & - & $25 \pm 8(\uparrow \approx)$ & $21 \pm 9(\approx \downarrow)$ & $24 \pm 6(\uparrow \downarrow)$ & $22 \pm 11(\approx \downarrow)$ & $11 \pm 4(\approx \downarrow)$ & $13 \pm 8(\downarrow \downarrow)$ \\
\hline & $f C_{Y H L}$ & - & - & $0 \pm 0$ & $0 \pm 0$ & $0 \pm 0$ & $0 \pm 0$ & $0 \pm 0$ & $0 \pm 0$ \\
\hline & $f N_{Y L}$ & - & - & $-0 \pm 0$ & $3 \pm 0$ & $0 \pm 0$ & $1 \pm 0$ & $0 \pm 0$ & $2 \pm 1$ \\
\hline & $k_{Y L}$ & - & & $-25 \pm 11(\downarrow \downarrow)$ & $2 \pm 1$ & $24 \pm 13(\downarrow \downarrow)$ & $5 \pm 5(\downarrow \approx)$ & $15 \pm 10(\downarrow \downarrow)$ & $3 \pm 4$ \\
\hline & $k_{Y R}$ & - & - & $3 \pm 4(\approx \uparrow)$ & $0 \pm 0$ & $4 \pm 6(\approx \uparrow)$ & $2 \pm 2$ & $15 \pm 13(\uparrow \uparrow)$ & $12 \pm 8(\uparrow \uparrow)$ \\
\hline & $k_{Y H L}$ & - & - & $0 \pm 0$ & $0 \pm 0$ & $0 \pm 0$ & $0 \pm 0$ & $0 \pm 0$ & $0 \pm 0$ \\
\hline & $k_{P}$ & $0 \pm 0$ & $0 \pm 0$ & $0 \pm 0$ & $0 \pm 0$ & $0 \pm 0$ & $0 \pm 0$ & $0 \pm 0$ & $0 \pm 0$ \\
\hline & $k_{r m}$ & $21 \pm 5(\approx \uparrow)$ & $2 \pm 4(\approx \uparrow)$ & $14 \pm 6(\downarrow \approx)$ & $0 \pm 0$ & $11 \pm 7(\downarrow \approx)$ & $2 \pm 3$ & $25 \pm 3(\downarrow \approx)$ & $8 \pm 3(\downarrow \approx)$ \\
\hline & $\Sigma S_{i}$ & $85 \pm 1$ & $74 \pm 0$ & $89 \pm 4$ & $80 \pm 10$ & $88 \pm 3$ & $86 \pm 4$ & $87 \pm 2$ & $80 \pm 3$ \\
\hline
\end{tabular}

The higher the index, the higher is the sensitivity of the model outputs to the parameter. Italics values mean that parameters had an index higher than 10\% in at least one of the dates. A qualitative pattern of the temporal trend of the sensitivity index is given in brackets (first symbol: Day 3-44, second symbol: Day 44-189): increasing ( $\uparrow)$, decreasing ( $\downarrow)$, and constant value $(\approx)$. The table reports also the sum of the first sensitivity indices, in the last row dedicated to each model. *Each row reports the first-order sensitivity index $\left(S_{i}\right)$ of the parameter. At the end of each model, $\Sigma_{S i}$ is the sum of first order sensitivity indices for all model parameters. 
Table 6. Average differences between Sobol' total (STi) and first-order (Si) sensitivity indices (\%) calculated in seven dates over the period Day 3-189 ( \pm standard deviation).

\begin{tabular}{|c|c|c|c|c|c|c|c|c|c|}
\hline \multirow[t]{3}{*}{ Model } & \multirow[t]{3}{*}{ Parameter* } & \multicolumn{8}{|c|}{ Input } \\
\hline & & \multicolumn{2}{|c|}{ None (CON) } & \multicolumn{2}{|c|}{ Liquid manure (LM) } & \multicolumn{2}{|c|}{ Solid manure (SM) } & \multicolumn{2}{|c|}{ Low-N crop residue (CR) } \\
\hline & & $\mathrm{CO}_{2}$ & SMN & $\mathrm{Net} \mathrm{CO}_{2}$ & Net SMN & Net $\mathrm{CO}_{2}$ & Net SMN & Net $\mathrm{CO}_{2}$ & Net SMN \\
\hline \multirow[t]{13}{*}{ MOD $D_{\text {First }}$} & $f C_{Y R}$ soil & $9 \pm 1$ & $10 \pm 2(\approx \approx)$ & $0 \pm 0$ & $0 \pm 0$ & $0 \pm 0$ & $0 \pm 0$ & $1 \pm 1$ & $3 \pm 2$ \\
\hline & $k_{Y R}$ soil & $9 \pm 2(\approx \approx)$ & $12 \pm 4(\downarrow \downarrow)$ & $0 \pm 0$ & $0 \pm 0$ & $0 \pm 0$ & $0 \pm 0$ & $1 \pm 1$ & $3 \pm 2$ \\
\hline & $k_{0}$ & $0 \pm 0$ & $1 \pm 0$ & $0 \pm 0$ & $0 \pm 0$ & $0 \pm 0$ & $0 \pm 0$ & $0 \pm 0$ & $0 \pm 0$ \\
\hline & $f N_{B}$ & $0 \pm 0$ & $18 \pm 6(\downarrow \downarrow)$ & $0 \pm 0$ & $7 \pm 2$ & $10 \pm 2(\approx \approx)$ & $13 \pm 4(\uparrow \downarrow)$ & $12 \pm 2(\uparrow \approx)$ & $32 \pm 13(\uparrow \downarrow)$ \\
\hline & eff & $5 \pm 1$ & $11 \pm 2(\approx \approx)$ & $4 \pm 1$ & $5 \pm 1$ & $12 \pm 1(\approx \approx)$ & $12 \pm 5(\uparrow \approx)$ & $14 \pm 2(\approx \approx)$ & $33 \pm 15(\uparrow \downarrow)$ \\
\hline & $f_{B}$ & $0 \pm 0$ & $0 \pm 0$ & $0 \pm 0$ & $0 \pm 0$ & $0 \pm 0$ & $0 \pm 0$ & $0 \pm 0$ & $0 \pm 0$ \\
\hline & $k_{B}$ & $0 \pm 0$ & $2 \pm 1$ & $0 \pm 0$ & $1 \pm 1$ & $1 \pm 1$ & $2 \pm 3$ & $2 \pm 1$ & $7 \pm 7(\uparrow \uparrow)$ \\
\hline & $f C_{Y L}$ & - & - & $4 \pm 2$ & $5 \pm 4(\downarrow \approx)$ & $11 \pm 3(\approx \downarrow)$ & $9 \pm 3(\approx \downarrow)$ & $5 \pm 4(\downarrow \approx)$ & $7 \pm 5(\downarrow \approx)$ \\
\hline & $f C_{Y H L}$ & - & - & $0 \pm 0$ & $0 \pm 0$ & $0 \pm 0$ & $0 \pm 0$ & $0 \pm 0$ & $0 \pm 0$ \\
\hline & $f N_{Y L}$ & - & - & $0 \pm 0$ & $0 \pm 0$ & $0 \pm 0$ & $1 \pm 0$ & $0 \pm 0$ & $1 \pm 1$ \\
\hline & $k_{Y L}$ & - & - & $3 \pm 3$ & $4 \pm 6(\downarrow \approx)$ & $4 \pm 5(\downarrow \approx)$ & $3 \pm 4$ & $3 \pm 4(\downarrow \approx)$ & $2 \pm 3$ \\
\hline & $k_{Y R}$ & - & - & $1 \pm 2$ & $1 \pm 1$ & $2 \pm 2$ & $3 \pm 2$ & $9 \pm 4(\uparrow \approx)$ & $13 \pm 6(\uparrow \downarrow)$ \\
\hline & $k_{Y H L}$ & - & - & $0 \pm 0$ & $0 \pm 0$ & $0 \pm 0$ & $0 \pm 0$ & $0 \pm 0$ & $0 \pm 0$ \\
\hline \multirow{18}{*}{$\mathrm{MOD}_{\text {Monod }}$} & $f C_{Y R}$ soil & $8 \pm 4(\approx \approx)$ & $6 \pm 3(\approx \approx)$ & $1 \pm 1$ & $0 \pm 1$ & $1 \pm 1$ & $0 \pm 0$ & $1 \pm 1$ & $1 \pm 1$ \\
\hline & $k_{Y R}$ soil & $15 \pm 2(\uparrow \approx)$ & $12 \pm 1(\approx \approx)$ & $1 \pm 1$ & $1 \pm 1$ & $1 \pm 1$ & $0 \pm 0$ & $2 \pm 2$ & $2 \pm 1$ \\
\hline & $k_{0}$ & $0 \pm 0$ & $0 \pm 0$ & $0 \pm 0$ & $0 \pm 0$ & $0 \pm 0$ & $0 \pm 0$ & $0 \pm 0$ & $0 \pm 0$ \\
\hline & $f N_{B}$ & $0 \pm 0$ & $24 \pm 4(\approx \downarrow)$ & $0 \pm 0$ & $13 \pm 3(\downarrow \approx)$ & $9 \pm 3(\uparrow \approx)$ & $11 \pm 1(\approx \approx)$ & $11 \pm 5(\uparrow \approx)$ & $13 \pm 3(\uparrow \approx)$ \\
\hline & eff & $4 \pm 1$ & $13 \pm 1(\approx \approx)$ & $4 \pm 1$ & $9 \pm 1(\approx \approx)$ & $15 \pm 2(\uparrow \approx)$ & $10 \pm 3(\uparrow \approx)$ & $19 \pm 5(\uparrow \uparrow)$ & $15 \pm 8(\uparrow \approx)$ \\
\hline & $f_{B}$ & $0 \pm 0$ & $1 \pm 0$ & $0 \pm 0$ & $0 \pm 0$ & $0 \pm 0$ & $0 \pm 0$ & $0 \pm 0$ & $0 \pm 0$ \\
\hline & $k_{B}$ & $1 \pm 0$ & $4 \pm 0$ & $0 \pm 1$ & $1 \pm 2$ & $1 \pm 2$ & $2 \pm 2$ & $2 \pm 2$ & $5 \pm 5(\uparrow \uparrow)$ \\
\hline & $f C_{Y L}$ & - & - & $6 \pm 1$ & $10 \pm 7(\downarrow \approx)$ & $11 \pm 3(\uparrow \approx)$ & $9 \pm 3(\approx \downarrow)$ & $7 \pm 3(\approx \approx)$ & $7 \pm 4(\downarrow \downarrow)$ \\
\hline & $f C_{Y H L}$ & - & - & $0 \pm 0$ & $0 \pm 0$ & $0 \pm 0$ & $0 \pm 0$ & $0 \pm 0$ & $0 \pm 0$ \\
\hline & $f N_{Y L}$ & - & - & $0 \pm 0$ & $1 \pm 0$ & $0 \pm 0$ & $1 \pm 0$ & $0 \pm 0$ & $1 \pm 1$ \\
\hline & $k_{Y L}$ & - & - & $8 \pm 4(\downarrow \approx)$ & $9 \pm 7(\downarrow \approx)$ & $10 \pm 6(\downarrow \downarrow)$ & $8 \pm 6(\downarrow \approx)$ & $6 \pm 4(\downarrow \approx)$ & $5 \pm 4(\downarrow \approx)$ \\
\hline & $k_{Y R}$ & - & - & $2 \pm 2$ & $1 \pm 1$ & $2 \pm 2$ & $2 \pm 2$ & $8 \pm 6(\uparrow \approx)$ & $7 \pm 4(\uparrow \approx)$ \\
\hline & $k_{Y H L}$ & - & - & $0 \pm 0$ & $0 \pm 0$ & $0 \pm 0$ & $0 \pm 0$ & $0 \pm 0$ & $0 \pm 0$ \\
\hline & $k_{\text {mYR soil }}$ & $15 \pm 3(\uparrow \approx)$ & $14 \pm 1(\approx \approx)$ & $1 \pm 1$ & $1 \pm 1$ & $1 \pm 1$ & $0 \pm 0$ & $2 \pm 2$ & $2 \pm 1$ \\
\hline & $k_{m 0}$ & $0 \pm 0$ & $1 \pm 0$ & $0 \pm 0$ & $0 \pm 0$ & $0 \pm 0$ & $0 \pm 0$ & $0 \pm 0$ & $0 \pm 0$ \\
\hline & $k_{m Y L}$ & - & - & $6 \pm 5(\downarrow \approx)$ & $8 \pm 11(\downarrow \approx)$ & $6 \pm 6(\downarrow \approx)$ & $4 \pm 4(\downarrow \approx)$ & $6 \pm 6(\downarrow \approx)$ & $4 \pm 5(\downarrow \approx)$ \\
\hline & $k_{m Y R}$ & - & - & $2 \pm 2$ & $2 \pm 1$ & $2 \pm 2$ & $2 \pm 1$ & $9 \pm 6(\uparrow \approx)$ & $6 \pm 3(\uparrow \approx)$ \\
\hline & $k_{m Y H L}$ & - & - & $0 \pm 0$ & $0 \pm 0$ & $0 \pm 0$ & $0 \pm 0$ & $0 \pm 0$ & $0 \pm 0$ \\
\hline \multirow[t]{14}{*}{$\mathrm{MOD}_{\text {RevMonod }}$} & $f C_{Y R}$ soil & $8 \pm 1(\approx \approx)$ & $6 \pm 1$ & $0 \pm 0$ & $0 \pm 0$ & $0 \pm 0$ & $0 \pm 0$ & $1 \pm 1$ & $1 \pm 0$ \\
\hline & $k_{Y R}$ soil & $9 \pm 1$ & $7 \pm 0$ & $0 \pm 0$ & $0 \pm 0$ & $0 \pm 0$ & $0 \pm 0$ & $1 \pm 1$ & $1 \pm 1$ \\
\hline & $k_{0}$ & $0 \pm 0$ & $0 \pm 0$ & $0 \pm 0$ & $0 \pm 0$ & $0 \pm 0$ & $0 \pm 0$ & $0 \pm 0$ & $0 \pm 0$ \\
\hline & $f N_{B}$ & $0 \pm 0$ & $18 \pm 3(\approx \downarrow)$ & $0 \pm 0$ & $10 \pm 4(\downarrow \approx)$ & $6 \pm 2$ & $8 \pm 1$ & $7 \pm 3(\uparrow \approx)$ & $11 \pm 2(\approx \approx)$ \\
\hline & eff & $4 \pm 1$ & $11 \pm 0(\approx \approx)$ & $5 \pm 1$ & $7 \pm 1$ & $13 \pm 2(\uparrow \approx)$ & $7 \pm 2$ & $17 \pm 5(\uparrow \approx)$ & $12 \pm 6(\uparrow \approx)$ \\
\hline & $f_{B}$ & $0 \pm 0$ & $1 \pm 0$ & $0 \pm 0$ & $0 \pm 0$ & $0 \pm 0$ & $0 \pm 0$ & $0 \pm 0$ & $0 \pm 0$ \\
\hline & $k_{B}$ & $1 \pm 0$ & $5 \pm 0$ & $0 \pm 0$ & $1 \pm 1$ & $1 \pm 1$ & $1 \pm 2$ & $2 \pm 2$ & $4 \pm 4(\uparrow \uparrow)$ \\
\hline & $f C_{Y L}$ & - & - & $6 \pm 2$ & $9 \pm 6(\downarrow \approx)$ & $11 \pm 2(\approx \approx)$ & $9 \pm 3(\approx \approx)$ & $6 \pm 2$ & $6 \pm 3(\approx \downarrow)$ \\
\hline & $f C_{Y H L}$ & - & - & $0 \pm 0$ & $0 \pm 0$ & $0 \pm 0$ & $0 \pm 0$ & $0 \pm 0$ & $0 \pm 0$ \\
\hline & $f N_{Y L}$ & - & - & $0 \pm 0$ & $0 \pm 0$ & $0 \pm 0$ & $0 \pm 0$ & $0 \pm 0$ & $1 \pm 0$ \\
\hline & $k_{Y L}$ & - & - & $7 \pm 3(\downarrow \approx)$ & $9 \pm 7(\downarrow \approx)$ & $8 \pm 4(\downarrow \approx)$ & $7 \pm 6(\downarrow \approx)$ & $5 \pm 4(\downarrow \approx)$ & $5 \pm 5(\downarrow \approx)$ \\
\hline & $k_{Y R}$ & - & - & $1 \pm 1$ & $1 \pm 1$ & $1 \pm 1$ & $1 \pm 1$ & $6 \pm 5(\uparrow \approx)$ & $5 \pm 3$ \\
\hline & $k_{Y H L}$ & - & - & $0 \pm 0$ & $0 \pm 0$ & $0 \pm 0$ & $0 \pm 0$ & $0 \pm 0$ & $0 \pm 0$ \\
\hline & $k_{r m}$ & $9 \pm 1$ & $8 \pm 0$ & $5 \pm 3(\downarrow \approx)$ & $5 \pm 5(\downarrow \approx)$ & $5 \pm 3(\downarrow \approx)$ & $3 \pm 2$ & $12 \pm 2(\approx \approx)$ & $8 \pm 2$ \\
\hline \multirow[t]{14}{*}{$\mathrm{MOD}_{\text {First+Ove }}$} & $f C_{Y R}$ soil & $9 \pm 1$ & $10 \pm 2(\approx \approx)$ & $0 \pm 0$ & $0 \pm 0$ & $0 \pm 0$ & $0 \pm 0$ & $0 \pm 0$ & $3 \pm 2$ \\
\hline & $k_{Y R}$ soil & $9 \pm 2(\approx \approx)$ & $12 \pm 4(\downarrow \downarrow)$ & $0 \pm 0$ & $0 \pm 0$ & $0 \pm 0$ & $0 \pm 0$ & $0 \pm 0$ & $3 \pm 2$ \\
\hline & $k_{0}$ & $0 \pm 0$ & $1 \pm 0$ & $0 \pm 0$ & $0 \pm 0$ & $0 \pm 0$ & $0 \pm 0$ & $0 \pm 0$ & $0 \pm 0$ \\
\hline & $f N_{B}$ & $0 \pm 0$ & $18 \pm 6(\downarrow \downarrow)$ & $0 \pm 0$ & $7 \pm 2$ & $0 \pm 0$ & $11 \pm 4(\uparrow \downarrow)$ & $1 \pm 1$ & $30 \pm 13(\uparrow \downarrow)$ \\
\hline & eff & $5 \pm 1$ & $11 \pm 2(\approx \approx)$ & $4 \pm 2$ & $5 \pm 1$ & $4 \pm 1$ & $10 \pm 4(\uparrow \downarrow)$ & $5 \pm 0$ & $32 \pm 14(\uparrow \downarrow)$ \\
\hline & $f_{B}$ & $0 \pm 0$ & $0 \pm 0$ & $0 \pm 0$ & $0 \pm 0$ & $0 \pm 0$ & $0 \pm 0$ & $0 \pm 0$ & $0 \pm 0$ \\
\hline & $k_{B}$ & $0 \pm 0$ & $2 \pm 1$ & $0 \pm 0$ & $1 \pm 1$ & $0 \pm 0$ & $2 \pm 2$ & $0 \pm 0$ & $7 \pm 7(\uparrow \uparrow)$ \\
\hline & $f C_{Y L}$ & - & - & $4 \pm 2$ & $5 \pm 4(\downarrow \approx)$ & $5 \pm 2$ & $8 \pm 3(\approx \downarrow)$ & $2 \pm 2$ & $6 \pm 5(\downarrow \approx)$ \\
\hline & $f C_{Y H L}$ & - & - & $0 \pm 0$ & $0 \pm 0$ & $0 \pm 0$ & $0 \pm 0$ & $0 \pm 0$ & $0 \pm 0$ \\
\hline & $f N_{Y L}$ & - & - & $0 \pm 0$ & $0 \pm 0$ & $0 \pm 0$ & $1 \pm 0$ & $0 \pm 0$ & $1 \pm 1$ \\
\hline & $k_{Y L}$ & - & - & $3 \pm 3$ & $4 \pm 6(\downarrow \approx)$ & $3 \pm 3$ & $3 \pm 3$ & $2 \pm 3$ & $2 \pm 3$ \\
\hline & $k_{Y R}$ & - & - & $1 \pm 2$ & $1 \pm 1$ & $2 \pm 2$ & $3 \pm 2$ & $3 \pm 2$ & $12 \pm 6(\uparrow \downarrow)$ \\
\hline & $k_{Y H L}$ & - & - & $0 \pm 0$ & $0 \pm 0$ & $0 \pm 0$ & $0 \pm 0$ & $0 \pm 0$ & $0 \pm 0$ \\
\hline & $k_{P}$ & $0 \pm 0$ & $0 \pm 0$ & $0 \pm 0$ & $0 \pm 0$ & $0 \pm 0$ & $0 \pm 0$ & $0 \pm 0$ & $0 \pm 0$ \\
\hline
\end{tabular}


diminishing decomposition rates ( $\mathrm{N}$ inhibition hypothesis, Eq. 7) or by temporary storing excess- $\mathrm{C}$ in the polysaccharides pool (C-overflow mechanism, Eq. 8). In addition to eff, concentration of SMN (Tables 5 and 6) was also sensitive to a variation of $f N_{B}$ (and thus to the $\mathrm{C}$ to $\mathrm{N}$ ratio of soil microbial biomass). Together, eff and $f_{N B}$ explained from 26 to $63 \%$ of SMN variance; this is justified by their effect in determining $\mathrm{N}$ requirements by microbes (Eq. 4 ).

In the amended treatments, neither the most recalcitrant fraction of input $\mathrm{C}\left(f C_{Y H L}\right)$ nor its decomposition constant $\left(k_{Y H L}\right)$ gave effects on net $\mathrm{CO}_{2}$ emissions and SMN in all models. This can be attributed to range that we have chosen for $k_{Y H L}$ (Table 1) and to the reduced simulation time span that made the $Y_{H L}$ pool almost inert (data not shown). Conversely, $\mathrm{C}$ decomposition and SMN were very sensitive to variations of the size $\left(f C_{Y L}\right)$ and decomposition constant $\left(k_{Y L}\right)$ of the labile young pool $Y_{L}$ (Tables 5 and 6 ). Both parameters influence the mineralisation of labile $\mathrm{C}$; moreover, $f C_{Y L}$ is fundamental in regulating input- $\mathrm{N}$ availability by switching from $\mathrm{N}$-rich to $\mathrm{N}$-poor $Y_{L}$ pools (Tables 1 and 2), and thus strongly impacts SMN dynamics.

\section{Effects of carbon-overflow on $\mathrm{CO}_{2}$ emissions and net nitrogen mineralisation}

Differences between models implementing $\mathrm{N}$ inhibition hypothesis and C-overflow mechanism occurred in SM and CR (Figures 3A and $4 \mathrm{~A}$ ), but not LM (because for LM N was never limiting $\mathrm{C}$ decomposition). In SM and CR, models implementing C-overflow decomposed more input-C (on average $8 \%$ and $10 \%$ of added $\mathrm{C}$ at Day 189 in SM and $\mathrm{CR}$, respectively) and immobilised less $\mathrm{N}$ (on average $-0.7 \%$ and $-0.4 \%$ of added C at Day 189 in SM and CR, respectively) than models implementing the $\mathrm{N}$ inhibition hypothesis. The simulated dynamics of the polysaccharides pool were in agreement with that of Hadas et al. (1998). For a soil amended with a low-N straw (C to N ratio of 91), they simulated a net growth of the polysaccharides pool until $\mathrm{N}$ limitation persisted (Day 50-80). Thereafter, due to ceased accumulation, the polysaccharides declined, with a velocity depending on $k_{P}$. In our work net $\mathrm{C}$ accumulation in the polysaccharides pool lasted until Day 44-73 in SM and Day 189 in CR (Figure 5), when the maximum size of $P$ was reached. The maximum size of the polysaccharides pool (Figure 5) was

Table 6. Continued from previous page.

\begin{tabular}{|c|c|c|c|c|c|c|c|c|c|}
\hline \multirow[t]{3}{*}{ Model } & \multirow[t]{3}{*}{ Parameter* } & \multicolumn{8}{|c|}{ Input } \\
\hline & & \multicolumn{2}{|c|}{ None (CON) } & \multicolumn{2}{|c|}{ Liquid manure (LM) } & \multicolumn{2}{|c|}{ Solid manure (SM) } & \multicolumn{2}{|c|}{ Low-N crop residue (CR) } \\
\hline & & $\mathrm{CO}_{2}$ & SMN & Net $\mathrm{CO}_{2}$ & Net SMN & Net $\mathrm{CO}_{2}$ & Net SMN & Net $\mathrm{CO}_{2}$ & Net SMN \\
\hline \multirow{20}{*}{$\mathrm{MOD}_{\text {Monod+Over }}$} & $f C_{Y R}$ soil & $8 \pm 4(\uparrow \approx)$ & $6 \pm 3(\approx \approx)$ & $1 \pm 1$ & $0 \pm 1$ & $0 \pm 0$ & $0 \pm 0$ & $1 \pm 1$ & $1 \pm 1$ \\
\hline & $k_{Y R}$ soil & $15 \pm 2(\uparrow \approx)$ & $12 \pm 1(\approx \approx)$ & $1 \pm 1$ & $1 \pm 1$ & $1 \pm 1$ & $1 \pm 1$ & $1 \pm 1$ & $2 \pm 1$ \\
\hline & $k_{0}$ & $0 \pm 0$ & $0 \pm 0$ & $0 \pm 0$ & $0 \pm 0$ & $0 \pm 0$ & $0 \pm 0$ & $0 \pm 0$ & $0 \pm 0$ \\
\hline & $f N_{B}$ & $0 \pm 0$ & $24 \pm 4(\approx \downarrow)$ & $0 \pm 0$ & $12 \pm 3(\downarrow \approx)$ & $1 \pm 1$ & $10 \pm 2(\approx \approx)$ & $1 \pm 1$ & $12 \pm 3(\uparrow \downarrow)$ \\
\hline & eff & $4 \pm 1$ & $13 \pm 1(\approx \approx)$ & $4 \pm 1$ & $9 \pm 1(\approx \approx)$ & $6 \pm 1$ & $8 \pm 2(\approx \approx)$ & $7 \pm 2(\approx \approx)$ & $14 \pm 7(\uparrow \approx)$ \\
\hline & $f_{B}$ & $0 \pm 0$ & $1 \pm 0$ & $0 \pm 0$ & $0 \pm 0$ & $0 \pm 0$ & $0 \pm 0$ & $0 \pm 0$ & $0 \pm 0$ \\
\hline & $k_{B}$ & $1 \pm 0$ & $4 \pm 0$ & $0 \pm 1$ & $1 \pm 2$ & $0 \pm 1$ & $1 \pm 1$ & $0 \pm 1$ & $4 \pm 4(\uparrow \approx)$ \\
\hline & $f C_{Y L}$ & - & - & $6 \pm 1$ & $10 \pm 7(\downarrow \approx)$ & $6 \pm 1$ & $9 \pm 3(\approx \downarrow)$ & $4 \pm 3$ & $7 \pm 4(\downarrow \approx)$ \\
\hline & $f C_{Y H L}$ & - & $-0 \pm 0$ & $0 \pm 0$ & $0 \pm 0$ & $0 \pm 0$ & $0 \pm 0$ & $0 \pm 0$ & \\
\hline & $f N_{Y L}$ & - & $-0 \pm 0$ & $1 \pm 0$ & $0 \pm 0$ & $1 \pm 0$ & $0 \pm 0$ & $1 \pm 1$ & \\
\hline & $k_{Y L}$ & - & - & $8 \pm 4(\downarrow \approx)$ & $9 \pm 7(\downarrow \approx)$ & $9 \pm 5(\downarrow \approx)$ & $8 \pm 6(\downarrow \approx)$ & $5 \pm 4(\downarrow \approx)$ & $5 \pm 4(\downarrow \approx)$ \\
\hline & $k_{Y R}$ & - & - & $2 \pm 2$ & $1 \pm 1$ & $2 \pm 2$ & $3 \pm 2$ & $6 \pm 4(\uparrow \approx)$ & $7 \pm 4(\uparrow \approx)$ \\
\hline & $k_{Y H L}$ & - & - & $0 \pm 0$ & $0 \pm 0$ & $0 \pm 0$ & $0 \pm 0$ & $0 \pm 0$ & $0 \pm 0$ \\
\hline & $k_{P}$ & $0 \pm 0$ & $0 \pm 0$ & $0 \pm 0$ & $0 \pm 0$ & $0 \pm 0$ & $0 \pm 0$ & $0 \pm 0$ & $0 \pm 0$ \\
\hline & 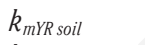 & $15 \pm 3(\uparrow \approx)$ & $14 \pm 1(\approx \approx)$ & $1 \pm 1$ & $1 \pm 1$ & $1 \pm 1$ & $0 \pm 1$ & $1 \pm 1$ & $2 \pm 1$ \\
\hline & $k_{m 0}$ & $0 \pm 0$ & $1 \pm 0$ & $0 \pm 0$ & $0 \pm 0$ & $0 \pm 0$ & $0 \pm 0$ & $0 \pm 0$ & $0 \pm 0$ \\
\hline & $k_{m Y L}$ & - & - & $6 \pm 5(\downarrow \approx)$ & $8 \pm 11(\downarrow \approx)$ & $5 \pm 5(\downarrow \approx)$ & $4 \pm 4(\downarrow \approx)$ & $5 \pm 6(\downarrow \approx)$ & $4 \pm 5(\downarrow \approx)$ \\
\hline & $k_{m Y R}$ & - & - & $2 \pm 2$ & $2 \pm 1$ & $2 \pm 2$ & $2 \pm 2$ & $7 \pm 5(\uparrow \approx)$ & $7 \pm 4(\uparrow \approx)$ \\
\hline & $k_{m Y H L}$ & - & - & $0 \pm 0$ & $0 \pm 0$ & $0 \pm 0$ & $0 \pm 0$ & $0 \pm 0$ & $0 \pm 0$ \\
\hline & $k_{m P}$ & $0 \pm 0$ & $0 \pm 0$ & $0 \pm 0$ & $0 \pm 0$ & $0 \pm 0$ & $0 \pm 0$ & $0 \pm 0$ & $0 \pm 0$ \\
\hline \multirow[t]{15}{*}{ MOD $D_{\text {RevMonod+Over }}$} & $f C_{Y R}$ soil & $8 \pm 1(\approx \approx)$ & $6 \pm 1$ & $0 \pm 0$ & $0 \pm 0$ & $0 \pm 0$ & $0 \pm 0$ & $0 \pm 0$ & $1 \pm 0$ \\
\hline & $k_{Y R}$ soil & $9 \pm 1$ & $7 \pm 0$ & $0 \pm 0$ & $0 \pm 0$ & $0 \pm 0$ & $0 \pm 0$ & $0 \pm 0$ & $1 \pm 1$ \\
\hline & $k_{0}$ & $0 \pm 0$ & $0 \pm 0$ & $0 \pm 0$ & $0 \pm 0$ & $0 \pm 0$ & $0 \pm 0$ & $0 \pm 0$ & $0 \pm 0$ \\
\hline & $f N_{B}$ & $0 \pm 0$ & $18 \pm 3(\approx \downarrow)$ & $0 \pm 0$ & $10 \pm 4(\downarrow \approx)$ & $0 \pm 0$ & $8 \pm 2$ & $0 \pm 0$ & $11 \pm 2(\approx \downarrow)$ \\
\hline & eff & $4 \pm 1$ & $11 \pm 0(\approx \approx)$ & $5 \pm 1$ & $7 \pm 1$ & $6 \pm 0$ & $6 \pm 1$ & $7 \pm 1$ & $12 \pm 6(\uparrow \approx)$ \\
\hline & $f_{B}$ & $0 \pm 0$ & $1 \pm 0$ & $0 \pm 0$ & $0 \pm 0$ & $0 \pm 0$ & $0 \pm 0$ & $0 \pm 0$ & $0 \pm 0$ \\
\hline & $k_{B}$ & $1 \pm 0$ & $5 \pm 0$ & $0 \pm 0$ & $1 \pm 1$ & $0 \pm 0$ & $1 \pm 1$ & $0 \pm 1$ & $4 \pm 4$ \\
\hline & $f C_{Y L}$ & - & - & $6 \pm 2$ & $9 \pm 6(\downarrow \approx)$ & $6 \pm 2$ & $8 \pm 3(\approx \downarrow)$ & $4 \pm 2$ & $6 \pm 3(\approx \downarrow)$ \\
\hline & $f C_{Y H L}$ & - & - & $0 \pm 0$ & $0 \pm 0$ & $0 \pm 0$ & $0 \pm 0$ & $0 \pm 0$ & $0 \pm 0$ \\
\hline & $f N_{Y L}$ & - & - & $0 \pm 0$ & $0 \pm 0$ & $0 \pm 0$ & $0 \pm 0$ & $0 \pm 0$ & $1 \pm 0$ \\
\hline & $k_{Y L}$ & - & - & $7 \pm 3(\downarrow \approx)$ & $9 \pm 7(\downarrow \approx)$ & $7 \pm 4(\downarrow \approx)$ & $7 \pm 6(\downarrow \approx)$ & $4 \pm 4(\downarrow \approx)$ & $5 \pm 4(\downarrow \approx)$ \\
\hline & $k_{Y R}$ & - & - & $1 \pm 1$ & $1 \pm 1$ & $1 \pm 1$ & $1 \pm 1$ & $4 \pm 3$ & $5 \pm 3$ \\
\hline & $k_{Y H L}$ & - & - & $0 \pm 0$ & $0 \pm 0$ & $0 \pm 0$ & $0 \pm 0$ & $0 \pm 0$ & $0 \pm 0$ \\
\hline & $k_{P}$ & $0 \pm 0$ & $0 \pm 0$ & $0 \pm 0$ & $0 \pm 0$ & $0 \pm 0$ & $0 \pm 0$ & $0 \pm 0$ & $0 \pm 0$ \\
\hline & $k_{r m}$ & $9 \pm 1$ & $8 \pm 0$ & $5 \pm 3(\downarrow \approx)$ & $5 \pm 5(\downarrow \approx)$ & $4 \pm 3$ & $3 \pm 2$ & $7 \pm 2$ & $8 \pm 2$ \\
\hline
\end{tabular}

The higher the difference, the higher is the importance of the interaction of other parameters with the parameter listed in the row. Italics values mean that parameters had a difference ST-S higher than 10\% in at least one of the dates. A qualitative pattern of the temporal trend of the differences is given in brackets (first symbol: Day 3-44, second symbol: Day 44-189): increasing $(\uparrow)$, decreasing $(\downarrow)$, and constant value $(\approx)$. 
similar for MOD $\mathrm{D}_{\text {First+Over }}$ and $\mathrm{MOD}_{\text {Monod+0ver }}(15-16 \%$ and $18-23 \%$ of input$\mathrm{C}$ in SM and CR, respectively) and was lower for MOD RevMonod+0ver $_{\text {(11\% }}$ and $9 \%$ of input-C in SM and CR, respectively). Results of MOD $\mathrm{D}_{\text {First+Over }}$ and $\mathrm{MOD}_{\text {Monod+Over }}$ are in agreement with those of Hadas et al. (1998) that reported a maximum accumulation of $\mathrm{C}$ in the pool $P$ of about 22 $25 \%$ of input-C.

However, sensitivity of $\mathrm{CO}_{2}$ emissions to the parameter describing polysaccharides decomposition rate $\left(k_{P}\right)$ was nil (Tables 5 and 6) because the amount of polysaccharides-C decomposed after Day 73 (Figure 5) was relatively low in SM (2-6\% of manure-C) and almost zero in CR. Thus, the effect of previously mentioned parameters (eff, $f C_{Y L}$ and $k_{Y L}$ ) masked that of $k_{P}$ in determining $\mathrm{CO}_{2}$ variation. Different results would have been obtained if all $\mathrm{C}$ in excess was respired as $\mathrm{CO}_{2}$, without a temporary storage in $P$ (Schimel and Weintraub, 2003; Neill and Gignuoux, 2006). However, the polysaccharides pool was specifically added to avoid strong increase of $\mathrm{C}$ respiration under $\mathrm{N}$-limited conditions (Hadas et al., 1998), even if both mechanisms (i.e., exudation of organic molecules and extra $\mathrm{CO}_{2}$ production) were proved to occur (Russel and Cook, 1995).

\section{Effects of decomposition kinetics on $\mathrm{CO}_{2}$ emissions and nitrogen mineralisation}

In models implementing classical and reverse Monod kinetics, $\mathrm{C}$ mineralisation, and to a less extent SMN, were sensitive to a variation of the half-saturation constants $k_{m Y R}$ soil, $k_{m Y L}$, and $k_{m Y R}\left(\mathrm{MOD}_{\text {Monod }}\right.$ and $\mathrm{MOD}_{\text {Monod+Over }}$ ), and $k_{r m}$ (MOD $\mathrm{DevMonod}_{\text {and }}$ MOD $\mathrm{D}_{\text {RevMonod+Over }}$ (Tables 5 and 6) due to their direct effect on $\mathrm{C}$ decomposition rates (Eqs. 2 and 3).

In the first days of incubation (until Day 7, 14 and 29 in LM, SM and $\mathrm{CR}$, respectively) the general impact of decomposition kinetics on $\mathrm{CO}_{2}$ emissions was similar for all inputs, and consisted in a reduction (on average by 0.5 ) of $\mathrm{C}$ mineralisation with Monod and reverse Monod compared to first-order kinetics (Figures 2A, 3A, and 4A). Reduced C decomposition induced less $\mathrm{N}$ mineralisation or immobilisation (Figures 2B, 3B, and 4B).

After Day 73, differences among models depended on input type and on the amount of decomposed $\mathrm{C}$ and $\mathrm{N}$. When microbial biomass grows without long-term $\mathrm{N}$ limitations, models with classical Monod kinetics decomposed more $\mathrm{C}$ and $\mathrm{N}$ than the other models. This was evident in LM and SM, excluding those simulations where $\mathrm{CO}_{2}$ emissions were very low $\left(10^{\text {th }}\right.$ percentile), when $\mathrm{C}$ mineralisation was on average $+6 \%$ and $+10 \%$ of manure-C in models with classical Monod kinetics compared to first-order and reverse Monod kinetics, respectively (Figures $2 \mathrm{~A}$ and $3 \mathrm{~A}$ ). The increase in $\mathrm{CO}_{2}$ emissions in $\mathrm{MOD}_{\text {Monod }}$ and $\mathrm{MOD}_{\text {Monod+Over }}$ was due to the growth of soil microbial biomass that in turn enhanced $\mathrm{C}$ decomposition rates $\left(k^{\prime} \times C_{B}, \mathrm{Eq} .2\right)$, while the effect of substrate exhaustion $\left(C_{S} /\left(k_{m}+C_{S}\right)\right.$, Eq. 2) was lower. Therefore, after Day 73 , NNM was higher (on average $+0.6 \%$ of manure-C) in models implementing classical Monod kinetics than in others, in those cases when input decomposition did not induce long-term net $\mathrm{N}$ immobilisation (Figure 2B and 3B).

Conversely, when substrate decomposed slowly, and microbial biomass was $\mathrm{C}$ - or $\mathrm{N}$ - limited (as for $\mathrm{CR}$ ), models with first-order kinetics decomposed more $\mathrm{C}$ (on average $8 \%$ of manure-C at Day 189) and immobilised more N (on average 1.0\% of manure-C at Day 189) than models with Monod and reverse Monod kinetics.

An extreme case occurred for $\mathrm{MOD}_{\text {First }}$ that showed negative net $\mathrm{CO}_{2}$ emissions after Day 29. This result was an artefact of model parametrization. In these simulations native soil pools $\left(Y_{R}\right.$ soil and $O$ ) had a $\mathrm{C}$ to $\mathrm{N}$ ratio higher than $C / \mathrm{N}_{\text {crit }}$ (Eq. 5) and thus induced net $\mathrm{N}$ immobilisation during decomposition. The application of CR to soil caused additional $\mathrm{N}$ immobilisation and generated the onset of $\mathrm{N}$ limitation. This did not happen in the unamended soil, which therefore released more $\mathrm{CO}_{2}$ than the treated soil.

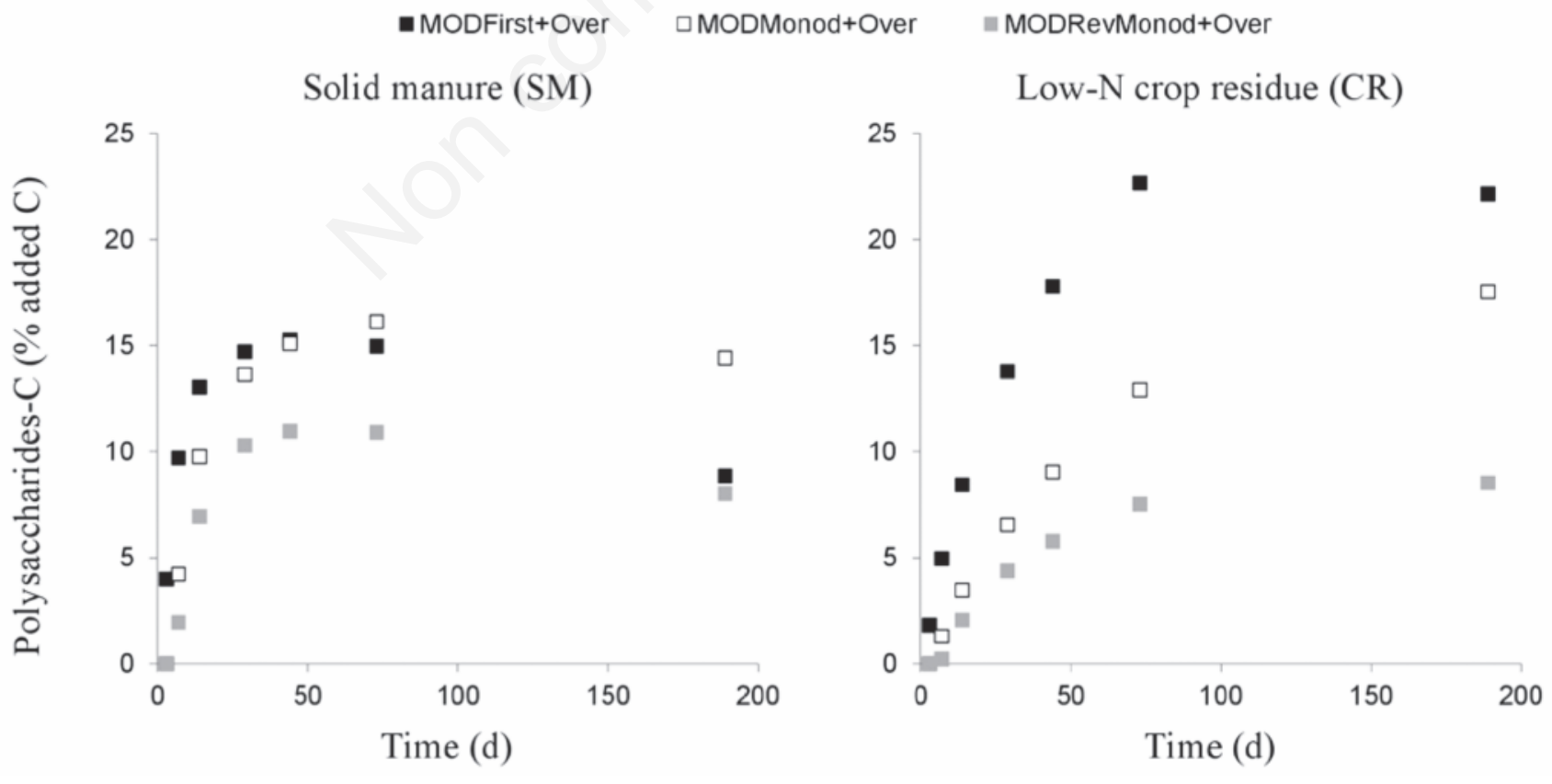

Figure 5. Simulated dynamics of net $\mathrm{C}$ in the polysaccharide pools (CP) for models implementing the C-overflow mechanism. Results refer to 90th percentile of $\mathrm{CP}$ distributions obtained during sensitivity analyses, and correspond to simulations in which shortage of $\mathrm{N}$ occurred. 


\section{Simulation of priming effects}

A very important finding is that some of the models were able to simulate negative or positive priming effects (PE). Indeed differences among models for net $\mathrm{CO}_{2}$ emissions and net $\mathrm{SMN}$ were not only due to a different turnover of input pools but also of native soil pools.
In $\mathrm{SM}$ and $\mathrm{CR}$, when the $\mathrm{C}$ to $\mathrm{N}$ ratio of $Y_{R}$ soil was higher than $C / N_{\text {crit }}$ and SM and CR exhausted the available mineral $\mathrm{N}$ causing $\mathrm{N}$ limitation, the models implementing the $\mathrm{N}$ inhibition hypothesis decomposed less $Y_{R}$ soil than in CON. This negative PE was small for models implementing Monod kinetics ( -1 and $-3 \%$ of added C at Day 189 in SM and CR, respectively), while it was higher for $\mathrm{MOD}_{\text {First }}(-10 \%$ and -

A

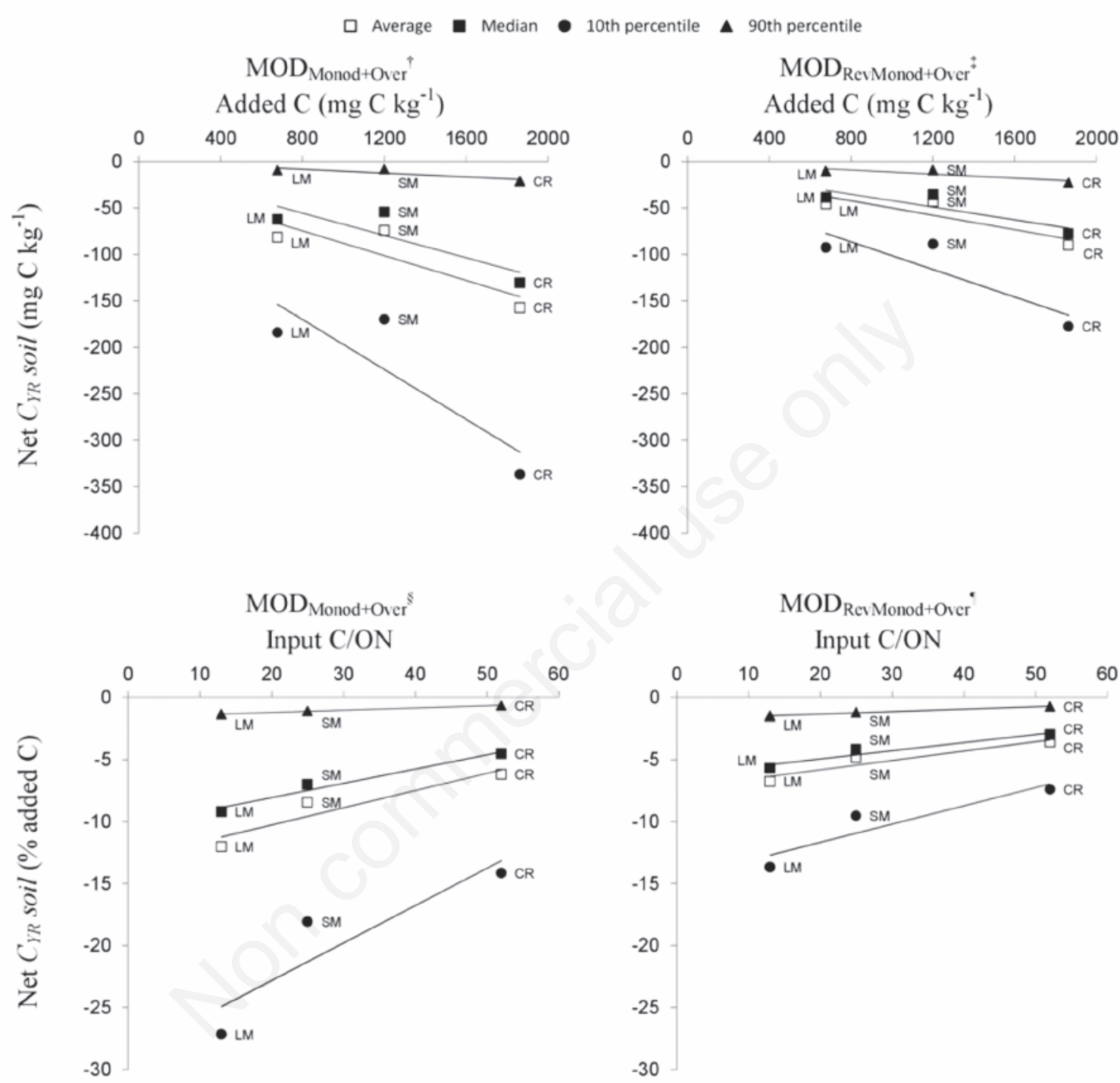

${ }^{4}$ Regression equations are Net $C_{Y R}$ soil $_{\text {cuverage }}=-0.07 \mathrm{C} / \mathrm{ON}-21.16\left(\mathrm{R}^{2}=0.74\right)$; Net $C_{Y R}$ soil $l_{\text {median }}=-0.06 \mathrm{C} / \mathrm{ON}-7.47$ $\left(\mathrm{R}^{2}=0.73\right)$; Net $C_{Y R}$ soil $l_{10 \text { th perc }}=-0.13 \mathrm{C} / \mathrm{ON}-62.92\left(\mathrm{R}^{2}=0.74\right)$; Net $C_{Y R}$ soil $l_{90 \text { th perc }}=-0.01 \mathrm{C} / \mathrm{ON}+0.03\left(\mathrm{R}^{2}=1.00\right)$

${ }^{*}$ Regression equations are Net $C_{Y R}$ soil $_{\text {average }}=-0.04 \mathrm{C} / \mathrm{ON}-11.31\left(\mathrm{R}^{2}=0.76\right)$; Net $C_{7 R}$ soil $l_{\text {median }}=-0.03 \mathrm{C} / \mathrm{ON}-7.77$ $\left(\mathrm{R}^{2}=0.75\right)$; Net $C_{Y R}$ soil $_{10 \text { th perc }}=-0.07 \mathrm{C} / \mathrm{ON}-26.91\left(\mathrm{R}^{2}=0.78\right)$; Net $C_{Y R}$ soil 90 th perc. $=-0.01 \mathrm{C} / \mathrm{ON}-0.43\left(\mathrm{R}^{2}=0.74\right)$.

${ }^{\S}$ Regression equations are: Net $C_{Y R}$ soil $_{\text {cverage }}=0.14 \mathrm{C} / \mathrm{ON}-13.05\left(\mathrm{R}^{2}=0.88\right)$; Net $C_{7 R}$ soil $l_{\text {median }}=0.12 \mathrm{C} / \mathrm{ON}-10.36$ $\left(\mathrm{R}^{2}=0.97\right)$; Net $C_{Y R}$ soil $_{10 \text { th perc }}=0.30 \mathrm{C} / \mathrm{ON}-28.86\left(\mathrm{R}^{2}=0.82\right)$; Net $C_{I R}$ soil $l_{90 \text { th perc }}=0.02 \mathrm{C} / \mathrm{ON}-1.59\left(\mathrm{R}^{2}=1.00\right)$.

'Regression equations are: Net $C_{Y R}$ soil cverage $=0.08 \mathrm{C} / \mathrm{ON}-7.32\left(\mathrm{R}^{2}=0.89\right)$; Net $C_{Y R}$ soil $l_{\text {median }}=0.07 \mathrm{C} / \mathrm{ON}-6.24\left(\mathrm{R}^{2}=0.92\right)$;

Net $C_{Y R}$ soil $_{10 \text { th perc }}=0.15 \mathrm{C} / \mathrm{ON}-14.61\left(\mathrm{R}^{2}=0.85\right)$; Net $C_{Y R}$ soil ${ }_{90 \text { th perc }}=0.02 \mathrm{C} / \mathrm{ON}-1.74\left(\mathrm{R}^{2}=0.99\right)$.

Figure 6. Priming effect simulated by the models $\mathrm{MOD}_{\text {Monod+Ove }} \mathrm{r}$ and $\left.\mathrm{MOD} \mathrm{D}_{\text {RevMonod+Over. }} \mathrm{A}\right)$ Linear regressions between added $\mathrm{C}$ and net $\mathrm{C}$ in the soil resistant pool $\left(C_{Y R}\right.$ soil) at Day 189. B) Linear regressions between $\mathrm{C}$ to organic $\mathrm{N}$ ratio of the input $(\mathrm{C} / O N)$ and net $C_{Y R}$ soil at Day 189. Net $C_{Y R}$ soil is the size of the simulated CYR soil in the amended treatments minus the simulated $C_{Y R}$ soil in the unamended control treatment. The four lines in each graph describe four statistical distributions of $C_{Y R}$ soil. Each line is made of three points, each corresponding to an amendment: LM, liquid manure; SM, solid manure; CR, crop residue. 
21\%). In addition, models implementing Monod kinetics (irrespective of $\mathrm{N}$ control mechanism on $\mathrm{C}$ decomposition), always showed positive PEs on $Y_{R}$ soil when decomposition of native SOM was not N-limited. This is represented in Figure 6 (only for models with C-overflow) with negative net $C_{Y R}$ soil, i.e., less $C_{Y R}$ soil at the end of the incubation in the amended treatments than in $\mathrm{CON}$.

Increasing the amount of added-C, more $Y_{R}$ soil was decomposed (Figure 6A), in particular for LM and SM. Decomposition of $Y_{R}$ soil increased as the input $\mathrm{C}$ to organic $\mathrm{N}$ ratio decreased (Figure $6 \mathrm{~B}$ ); this was in accordance with the assumption that as more input-C was decomposed (in the order $\mathrm{LM}>\mathrm{SM}>\mathrm{CR}$ ), more microbial biomass grew and thus more $Y_{R}$ soil was mineralised. Thus, in this case, the input $\mathrm{C}$ to organic $\mathrm{N}$ ratio is a proxy of input decomposability.

Moreover, distribution statistics of net $Y_{R}$ soil $\mathrm{C}$ showed that PEs varied from few percent points to $-27 \%$ and $-14 \%$ of added $C$, for models implementing classic and reverse Monod kinetics, respectively (Figure $6 \mathrm{~B})$. Variability of PE within each added input was also due to differences in decomposability, resulting from the partitioning of added $\mathrm{C}$ and $\mathrm{N}$ among young pools: high $\mathrm{C}$ decomposition induced high microbial growth that decomposed more $Y_{R}$ soil, while low, often N-limited, microbial growth did not stimulate $Y_{R}$ soil decomposition, and thus did not produce appreciable PEs.

In addition, higher $\mathrm{C}$ decomposition in models implementing classical Monod than reverse Monod kinetics (Figures 2A, 3A and 4A) gave higher PEs (Figure 6).

Models implementing Monod kinetics predicted PE according to cometabolism of fresh $\left(Y_{L}, Y_{R}\right)$ and native $\left(Y_{R}\right.$ soil) organic matter (Kuzyakov et al., 2000; Wang et al., 2015), under constraints of stoichiometric decomposition theory (Hessen et al., 2004; Craine et al., 2007; Chen et al., 2014). According to this theory, high-quality substrates (high accessibility, fast decomposition, and with $\mathrm{C}$ to $\mathrm{N}$ ratio close to $C / N_{\text {crit }}$ ) induce balanced microbial growth that in turn enhances decomposition of native SOM. Our results were in agreement with this theory. The size of PE was lower in CR compared to LM and SM, due to lower decomposition rates and higher $\mathrm{C}$ to $\mathrm{N}$ ratio of $\mathrm{CR}$. Moreover, $\mathrm{PE}$ was lower in simulations when microbial biomass was $\mathrm{C}$ or $\mathrm{N}$-limited following LM, SM or CR additions (Figure 6B).

Our models do not implement the mechanisms of priming effect proposed by two published theories, and thus our results are not in agreement with these theories. First, predicted PEs (mg C kg-1) increased linearly with $\mathrm{C}$ addition (Figure $6 \mathrm{~A}$ ). While this is in accordance with experimental results for low $\mathrm{C}$ additions, a negative relationship was demonstrated between applied $\mathrm{C}$ and primed $\mathrm{C}$ when the ratio of added- $\mathrm{C}$ to microbial biomass $\mathrm{C}$ exceeds about 50 (high $\mathrm{C}$ additions), possibly due to preferential substrate utilisation (Blagodatskaya and Kuzyakov, 2008). The preferential substrate decomposition theory (Wang et al., 2015) states that a negative PE might occur when microorganisms preferentially decompose the added organic materials, rather than the native soil organic matter. In our analysis, models did not predict this negative PE because no preferential decomposition mechanisms are implemented in our models.

Second, predicted PE was not in agreement with the microbial Nmining theory (Moorhead and Sinsabaugh, 2006; Craine et al., 2007; Chen et al., 2014; Wang et al., 2015), stating that soil microbial biomass feeds on added fresh organic matter to get energy, and decomposes native SOM to release limiting nutrients $(\mathrm{N})$. Based on this theory, we should have obtained higher PE when the shortage of $\mathrm{N}$ increased (i.e., in CR compared to manures, and in simulations with $\mathrm{N}$ shortage). However, our models do not implement differential mechanisms for $\mathrm{C}$ and $\mathrm{N}$ mineralisation, and thus this effect could not be obtained.

\section{Conclusions}

Model modifications (introduction of C-overflow and Monod decomposition kinetics) had an effect on simulated $\mathrm{CO}_{2}$ emissions, soil mineral nitrogen concentration, and turnover of added (manures and crop residues) and native soil organic matter pools. In particular, models implementing Monod decomposition kinetics were able to simulate positive priming effects after application of all inputs. Moreover, sensitivity analysis indicated that simulated $\mathrm{CO}_{2}$ and $\mathrm{SMN}$ were sensitive to a variation of only few model parameters; these are the parameters that should be selected for model calibration using experimental measurements.

\section{References}

Acutis M, Ducco G, Grignani C, 2000. Stochastic use of the LEACHN model to forecast nitrate leaching in different maize cropping systems. Eur. J. Agron. 13:191-206.

Allison SD, Wallenstein MD, Bradford MA, 2010. Soil-carbon response to warming dependent on microbial physiology. Nat. Geosci. 3:336-40.

Bechini L, Marino P, 2009. Short-term nitrogen fertilizing value of liquid dairy manures is mainly due to ammonium. Soil Sci. Soc. Am. J. 73:2159-69.

Blagodatsky S, Blagodatskaya E, Yuyukina T, Kuzyakov Y, 2010. Model of apparent and real priming effects: linking microbial activity with soil organic matter decomposition. Soil Biol. Biochem. 42:1275-83.

Blagodatskaya E, Kuzyakov Y, 2008. Mechanisms of real and apparent priming effects and their dependence on soil microbial biomass and community structure: critical review. Biol. Fert. Soils 45:115-131.

Blagodatsky SA, Richter 0, 1998. Microbial growth in soil and nitrogen turnover: a theoretical model considering the activity state of microorganisms. Soil Biol. Biochem. 30:1743-55.

Blagodatsky SA, Yevdokimov IV, Larionova AA, Richter J, 1998. Microbial growth in soil and nitrogen turnover: model calibration with laboratory data. Soil Biol. Biochem. 30:1757-64.

Calderón FJ, McCarty GW, Reeves JB, 2005. Analysis of manure and soil nitrogen mineralization during incubation. Biol. Fertil. Soils 41:328-36.

Cariboni J, Gatelli D, Liska R, Saltelli A, 2007. The role of sensitivity analysis in ecological modelling. Ecol. Model. 203:167-82.

Cavalli D, Bechini L, 2011. Sensitivity analysis and calibration of CN-SIM to simulate the mineralisation of liquid dairy manures. Soil Biol. Biochem. 43:1207-19.

Cavalli D, Bechini L, 2012. Multi-objective optimisation of a model of the decomposition of animal slurry in soil: Tradeoffs between simulated C and N dynamics. Soil Biol. Biochem. 48:113-24.

Cavalli D, Bechini L, Marino P, 2014. Measuring and modeling soil carbon respiration following repeated dairy slurry application. Soil Sci. Soc. Am. J. 78:1414-25.

Cavalli D, Cabassi G, Borrelli L, Geromela G, Bechini L, Degano L, Marino Gallina P, 2015. Nitrogen fertilizer replacement value of undigested liquid cattlemanure and digestates. Eur. J. Agron. 73:34-41.

Chan K, Saltelli A, Tarantola S, 1997. Sensitivity analysis of model output: variance-based methods make the difference. pp 261-268 in S. Andradottir, K.J. Healy, H. Withers, B.L. Nelson (Eds.), Proce. 1997 Winter Simulation Conference, Atlanta, Georgia, USA.

Chen R, Senbayram M, Blagodatsky S, Myachina 0, Dittert K, Lin X, Blagodatskaya E, Kuzyakov Yakov, 2014. Soil C and N availability deter- 
mine the priming effect: microbial $\mathrm{N}$ mining and stoichiometric decomposition theories. Glob. Change Biol. 20:2356-67.

Cleveland CC, Liptzin D, 2007. C:N:P stoichiometry in soil: is there a "Redfield ratio" for the microbial biomass? Biogeochemistry 85:235-52.

Conant RT, Ryan MG, Ågren GI, Birge HE, Davidson EA, Eliasson PE, Evans SE, Frey SD, Giardina CP, Hopkins FM, Hyvönen R, Kirschbaum MUF, Lavallee JM, Leifeld J, Parton WJ, Steinweg JM, Wallenstein MD, Wetterstedt JÅM, Bradford MA, 2011. Temperature and soil organic matter decomposition rates-synthesis of current knowledge and a way forward. Glob. Change Biol. 17:3392-404.

Coucheney E, Buis S, Launay M, Constantin J, Mary B, de Cortázar-Atauri IG, Ripoche D, Beaudoin N, Ruget F, Andrianarisoa KS, Le Bas C, Justes E, Léonard J, 2015. Accuracy, robustness and behaviour of the STICS soil-crop model for plant, water and nitrogen outputs: Evaluation over a wide range of agro-environmental conditions in France. Environ. Model. Softw. 64:177-90.

Craine JM, Morrow C, Fierer N, 2007. Microbial nitrogen limitation increases decomposition. Ecology 88:2105-13.

Davidson EA, Janssens IA, 2006. Temperature sensitivity of soil carbon decomposition and feedbacks to climate change. Nature 440:165-73.

Delin S, Stenberg B, Nyberg A, Brohede L, 2012. Potential methods for estimating nitrogen fertilizer value of organic residues. Soil Use Manage. 28:283-91.

Friedel JK, Gabel D, 2001. Microbial biomass and microbial C:N ratio in bulk soil and buried bags for evaluating in situ net $\mathrm{N}$ mineralization in agricultural soils. J. Plant Nutr. Soil Sci. 164:673-9.

Gijsman AJ, Hoogenboom G, Parton WJ, Kerridge PC, 2002. Modifying DSSAT crop models for low-input agricultural systems using a soil organic matter-residue module from CENTURY. Agron. J. 94:462-74.

Grace PR, Ladd JN, Robertson P, Gage SH, 2006. SOCRATES-A simple model for predicting long-term changes in soil organic carbon in terrestrial ecosystems. Soil Biol. Biochem. 38:1172-6.

Griffiths BS, Spilles A, Bonkowski M, 2012. C:N:P stoichiometry and nutrient limitation of the soil microbial biomass in a grazed grassland site under experimental P limitation or excess. Ecol. Proc. 1:6.

Hadas A, Kautsky L, Goek M, Kara EE, 2004. Rates of decomposition of plant residues and available nitrogen in soil, related to residue composition through simulation of carbon and nitrogen turnover. Soil Biol. Biochem. 36:255-66.

Hadas A, Parkin TB, Stahl PD, 1998. Reduced $\mathrm{CO}_{2}$ release from decomposing wheat straw under $\mathrm{N}$-limiting conditions: simulation of carbon turnover. Eur. J. Soil Sci. 49:487-94.

Hassink J, 1994. Effect of soil texture on the size of the microbial biomass and on the amount of $\mathrm{C}$ and $\mathrm{N}$ mineralized per unit of microbial biomass in dutch grassland soils. Soil Biol. Biochem. 26:1573-81.

Henriksen TM, Breland TA, 1999. Evaluation of criteria for describing crop residue degradability in a model of carbon and nitrogen turnover in soil. Soil Biol. Biochem. 31:1135-49.

Hessen D0, Ågren GI, Anderson TR, Elser JJ, de Ruiter PC, 2004. Carbon sequestration in ecosystems: the role of stoichiometry. Ecology 85:1179-92.

Ingwersen J, Poll C, Streck T, Kandeler E, 2008. Micro-scale modelling of carbon turnover driven by microbial succession at a biogeochemical interface. Soil Biol. Biochem. 40:864-78.

Izaurralde RC, Williams JR, McGill WB, Rosenberg NJ, Quiroga Jakas MC, 2006. Simulating soil $\mathrm{C}$ dynamics with EPIC: model description and testing against long-term data. Ecol. Model. 192:362-84.

Jensen LS, Salo T, Palmason F, Breland TA, Henriksen TM, Stenberg B, Pedersen A, Lundström C, Esala M, 2005. Influence of biochemical quality on $\mathrm{C}$ and $\mathrm{N}$ mineralisation from a broad variety of plant materials in soil. Plant Soil 273:307-26.

Kätterer T, Andrén 0, 2001. The ICBM family of analytically solved models of soil carbon, nitrogen and microbial biomass dynamics - descriptions and application examples. Ecol. Model. 136:197-207.

Kuzyakov Y, 2010. Priming effects: interaction between living and dead organic matter. Soil Biol. Biochem. 42:1363-71.

Kuzyakov Y, Friedel JK, Stahr K, 2000. Review of mechanisms and quantification of priming effects. Soil Biol. Biochem. 32:1485-98.

Lawrence CR, Neff JC, Schimel JP, 2009. Does adding microbial mechanisms of decomposition improve soil organic matter models? A comparison of four models using data from a pulsed rewetting experiment. Soil Biol. Biochem. 41:1923-34.

Liang J, Li D, Shi Z, Tiedje JM, Zhou J, Schuur EAG, Konstantinidis KT, Luo Y, 2015. Methods for estimating temperature sensitivity of soil organic matter based on incubation data: a comparative evaluation. Soil Biol. Biochem. 80:127-35.

Manzoni S, Porporato A, 2007. A theoretical analysis of nonlinearities and feedbacks in soil carbon and nitrogen cycles. Soil Biol. Biochem. 39:1542-56.

Manzoni S, Porporato A, 2009. Soil carbon and nitrogen mineralization: theory and models across scales. Soil Biol. Biochem. 41:1355-79.

Moorhead DL, Sinsabaugh RL, 2006. A theoretical model of litter decay and microbial interaction. Ecol. Monogr. 76:151-74.

Morvan T, Nicolardot B, Péan L, 2006. Biochemical composition and kinetics of $\mathrm{C}$ and $\mathrm{N}$ mineralization of animal wastes: A typological approach. Biol. Fertil. Soils 42:513-22.

Müller C, Rütting T, Kattge J, Laughlin RJ, Stevens RJ, 2007. Estimation of parameters in complex ${ }^{15} \mathrm{~N}$ tracing models by Monte Carlo sampling. Soil Biol. Biochem. 39:715-26.

Neill C, Gignoux J, 2006. Soil organic matter decomposition driven by microbial growth: A simple model for a complex network of interactions. Soil Biol. Biochem. 38: 803-11.

Nicolardot B, Recous S, Mary B, 2001. Simulation of C and N mineralisation during crop residue decomposition: A simple dynamic model based on the C:N ratio of the residues. Plant Soil 228:83-103.

Peters K, Jensen LS, 2011. Biochemical characteristics of solid fractions from animal slurry separation and their effects on $\mathrm{C}$ and $\mathrm{N}$ mineralisation in soil. Biol. Fertil. Soils 47:447-55.

Petersen BM, Berntsen S, Hansen S, Jensen LS, 2005a. CN-SIM-a model for the turnover of soil organic matter. I. Long-term carbon and radiocarbon development. Soil Biol. Biochem. 37:359-74.

Petersen BM, Jensen LS, Hansen S, Pedersen A, Henriksen TM, Sørensen P, Trinsoutrot-Gattin I, Berntsen S, 2005b. CN-SIM: a model for the turnover of soil organic matter. II. Short-term carbon and nitrogen development. Soil Biol. Biochem. 37:375-93.

Redin M, Recous S, Aita C, Dietrich G, Skolaude AC, Ludke WH, Schmatz R, Giacomini SJ, 2014. How the chemical composition and heterogeneity of crop residue mixtures decomposing at the soil surface affects C and N mineralisation? Soil Biol. Biochem. 78:65-75.

Russel JB, Cook GM, 1995. Energetics of bacterial growth: balance of anabolic and catabolic reactions. Microbiol. Rev. 59:48-62.

Saltelli A, 2002. Sensitivity analysis for importance assessment. Risk Anal. 22:579-90.

Saltelli A, Annoni P, Azzini I, Campolongo F, Ratto M, Tarantola S, 2010. Variance based sensitivity analysis of model output. Design and estimator for the total sensitivity index. Comput. Phys. Commun. 181:259-70.

Saltelli A, Tarantola S, Campolongo F, Ratto M, 2004. Sensitivity analysis in practice. A guide to assessing scientific models. John Wiley \& Sons, New York, NY, USA.

Schimel JP, Weintraub MN, 2003. The implication of exoenzyme activity on microbial carbon and nitrogen limitation in soil: a theoretical model. Soil Biol. Biochem. 35:549-63.

Sierra CA, Harmon ME, Perakis SS, 2011. Decomposition of heterogeneous organic matter and its long-term stabilization in soils. Ecol. Model. 81:619-34. 
Sierra CA, Malghani S, Müller M, 2015a. Model structure and parameter identification of soil organic matter models. Soil Biol. Biochem. 90:197-203.

Sierra CA, Müller M, 2015. A general mathematical framework for representing soil organic matter dynamics. Ecol. Monogr. 85:505-24.

Sierra CA, Trumbore SE, Davidson EA, Vicca S, Janssens I, 2015b. Sensitivity of decomposition rates of soil organic matter with respect to simultaneous changes in temperature and moisture. J. Adv. Model. Earth Syst. 7:335-56.

Sobol' IM, 1993. Sensitivity estimates for nonlinear mathematical models. Math. Model. Comput. Exp. 1:407-14.

Sobol' IM, Kucherenko SS, 2005. Global sensitivity indices for nonlinear mathematical models. Review. Wilmott Magazine 2:2-7.

Sørensen P, Fernández JA, 2003. Dietary effects on the composition of pig slurry and on the plant utilization of pig slurry nitrogen. J. Agr. Sci. 140:343-55.

Sørensen P, Weisbjerg MR, Lund P, 2003. Dietary effects on the composition and plant utilization of nitrogen in dairy cattle manure. J. Agric. Sci. 141:79-91.

Thomsen IK, Olesen JE, 2000. C and N mineralization of composted and anaerobically stored ruminant manure in differently textured soils. J. Agric. Sci. Camb. 135:151-9.

Trinsoutrot I, Recous S, Bentz B, Linères M, Chèneby D, Nicolardot B, 2000. Biochemical quality of crop residues and carbon and nitrogen mineralization kinetics under nonlimiting nitrogen conditions. Soil Sci. Soc. Am. J. 64:918-26.

Van Kessel JS, Reeves III JB, Meisinger JJ, 2000. Nitrogen and carbon mineralisation of potential manure compounds. J. Environ. Qual. 29:1669-77.

Van Veen JA, Ladd JN, Amato M, 1985. Turnover of carbon and nitrogen through the microbial biomass in a sandy loam and a clay soil incubated with $\left[{ }^{14} \mathrm{C}(\mathrm{U})\right]$ glucose and $\left[{ }^{15} \mathrm{~N}\right]\left(\mathrm{NH}_{4}\right)_{2} \mathrm{SO}_{4}$ under different moisture regimes. Soil Biol. Biochem. 17:747-56.

Verberne ELJ, Hassink J, de Willigen P, Groot JJR, Van Veen JA, 1990. Modelling organic matter dynamics in different soils. Neth. J. Agr. Sci. 38:221-38.

Wang F, Mladenoff DJ, Forrester JA, Keough C, Parton WJ, 2013. Global sensitivity analysis of a modified CENTURY model for simulating impacts of harvesting fine woody biomass for bioenergy. Ecol. Model. 259:16-23.

Wang H, Boutton TW, Xu W, Hu G, Jiang P, Bai E, 2015. Quality of fresh organic matter affects priming of soil organic matter and substrate utilization patterns of microbes. Sci. Rep. 5:10102.

Wang X, He X, Williams JR, Izaurralde RC, Atwood JD, 2005a. Sensitivity and uncertainty analyses of crop yields and soil organic carbon simulated with EPIC. Trans. ASAE 48:1041-54.

Wang X, Youssef MA, Skaggs RW, Atwood JD, Frankenberger JR, 2005b. Sensitivity analyses of the nitrogen simulation model, DRAINMOD-N II. Trans. ASAE 48:2205-12.

Wutzler T, Reichstein M, 2008. Colimitation of decomposition by substrate and decomposers - a comparison of model formulations. Biogeosciences 5:749-59.

Wutzler T, Reichstein M, 2013. Priming and substrate quality interactions in soil organic matter models. Biogeosciences 10:2089-103.

Zelenev V, van Bruggen AHC, Leffelaar PA, Bloem J, Semenov AM, 2006. Oscillating dynamics of bacterial populations and their predators in response to fresh organic matter added to soil: the simulation model 'BACWAVE-WEB'. Soil Biol. Biochem. 38:1690-711. 